University of Louisville

ThinkIR: The University of Louisville's Institutional Repository

Electronic Theses and Dissertations

$12-2001$

\title{
Characterization of secA-sod operon in Borrellia burgdorferi.
}

\author{
Tonya Lynn Nichols
}

University of Louisville

Follow this and additional works at: https://ir.library.louisville.edu/etd

Part of the Medical Immunology Commons, and the Medical Microbiology Commons

\section{Recommended Citation}

Nichols, Tonya Lynn, "Characterization of secA-sod operon in Borrellia burgdorferi." (2001). Electronic Theses and Dissertations. Paper 2881.

https://doi.org/10.18297/etd/2881

This Doctoral Dissertation is brought to you for free and open access by ThinkIR: The University of Louisville's Institutional Repository. It has been accepted for inclusion in Electronic Theses and Dissertations by an authorized administrator of ThinkIR: The University of Louisville's Institutional Repository. This title appears here courtesy of the author, who has retained all other copyrights. For more information, please contact thinkir@louisville.edu. 


\title{
CHARACTERIZATION OF THE SECA-SOD OPERON IN BORRELIA BURGDORFERI
}

\author{
By \\ Tonya Lynn Nichols \\ B. S., Livingston University, 1986 \\ M. S., Baylor University, 1991
}

\begin{abstract}
A Dissertation
Submitted to the Faculty of the

Graduate School of the University of Louisville

In Partial Fulfillment of the Requirements
\end{abstract}

For the Degree of

Doctor of Philosophy

Department of Microbiology and Immunology

University of Louisville School of Medicine

Louisville, Kentucky 


\section{CHARACTERIZATION OF THE SECA-SOD OPERON IN BORRELIA BURGDORFERI}

\section{By}

Tonya Lynn Nichols

B. S., Livingston University, 1986

M. S., Baylor University, 1991

A Dissertation Approved on

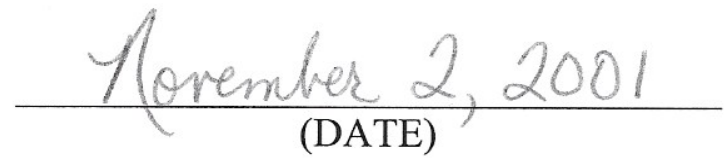

By the Following Reading Committee:

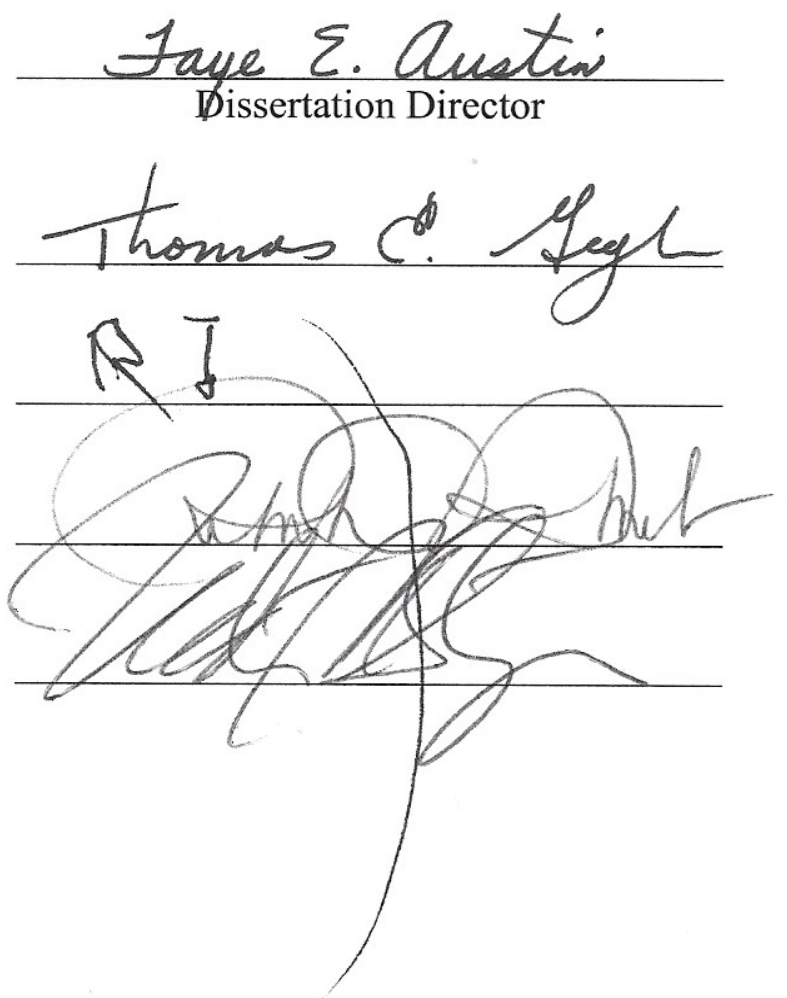




\begin{abstract}
Borrelia burgdorferi, the causative agent of Lyme disease, has been characterized as a microaerophilic spirochete. $\mathrm{O}_{2}$ consumption and utilization potentially yield reactive oxygen intermediates, such as superoxide, hydroxyl radicals, and hydrogen peroxide. This study investigated the expression of the sod gene, which encodes the only, identified oxidative defense mechanism in B. burgdorferi. Using primer extension analysis and RTPCR, it was found that sod and secA are organized as a single transcriptional unit under the control of $\sigma^{70}$-like promoter upstream of the secA open reading frame. Generally, gene expression decreases with increased distance from the promoter; however, secA expression was observed to be relatively lower amounts than sod. Both genes were expressed in all phases of growth, with greatest expression observed in stationary phase. Because transcription of most sod genes is tightly regulated by iron concentration, the secA-sod gene expression was analyzed in borrelial cells grown in varying amounts of metal. Ironrestriction did not significantly affect growth nor did it affect transcription of $\sec A$ or sod. Likewise, no major differences in transcription were observed with restricting or supplementing media with manganese. To test the possibility that the borrelial SOD may function with either iron or manganese as a cofactor as in cambialistic SODs, SOD activity was assayed and the highest activity was observed in increased manganese concentrations. Protein expression profiles of Sh-2-82 cultured in metal-defined media were investigated by one- and two-dimensional gel electrophoresis. Approximately 15 proteins were differentially expressed in response to metal concentration.
\end{abstract}




\section{ACKNOWLEDGMENTS}

There are many individuals who have played pivotal roles in helping me become the scientist and the person that I am today. As a graduate student at the University of Louisville, I was most influenced by my major professor, Dr. Faye Austin. I thank her for the opportunity to work in her laboratory on B. burgdorferi. Under her guidance, I received a good foundation in the difficult study of RNA genetics. From her, I have learned the value of attention to detail in experimental design, experimentation, and record-keeping, which will follow me throughout my scientific career. Dr. Uldis Streips has also been a key player in my success. I appreciate the time he has devoted to my project and me. I have good memories of our trips to WindRiver where scientific thoughts and ideas were exchanged in an informal, enjoyable manner. I also thank Dr. Robert Stout, Dr. Thomas Geoghegan, and Dr. Ronald Doyle, for their helpful suggestions during this project and their participation as committee members. Additionally, I thank Joan Gagel for her coordination efforts in the preparation of this dissertation. For her kindness and assistance, I would like to thank Jan Powars. There were many others who befriended me and supported me throughout my graduate study, and I am thankful for all of my friends at the University of Louisville. Most of all, I am grateful to my husband, Greg, and our two sons, Matthew and Joshua, for their love, support and understanding. Through a family effort, we have accomplished this goal together. 


\section{TABLE OF CONTENTS}

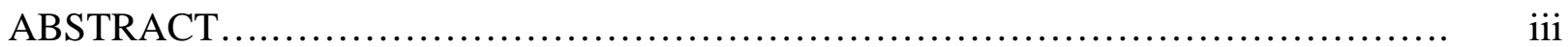

ACKNOWLEDGMENTS ......................................................... iv

TABLE OF CONTENTS ..........................................................

LIST OF TABLES..................................................................... viii

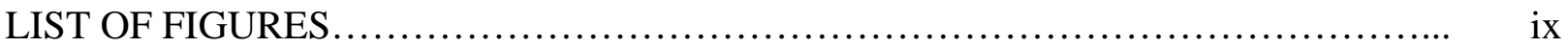

LIST OF ABBREVIATIONS........................................................ xi

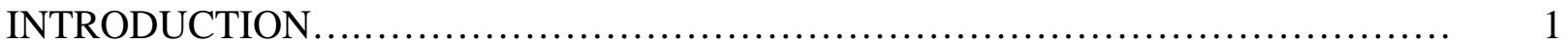

I. Lyme disease.................................................................... 1

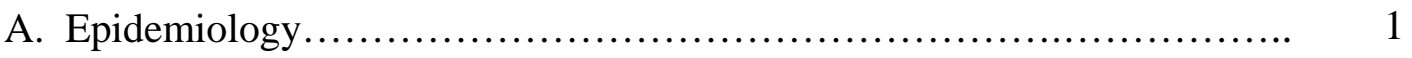

B. Clinical features...................................................... 4

C. Immunopathogenesis............................................. 6

D. Treatment................................................................ 11

E. Vaccine............................................................. 11

II. Borrelia burgdorferi........................................................ 13

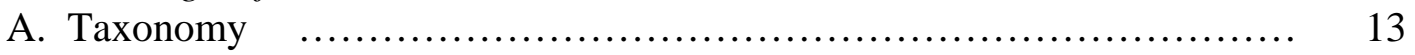

B. Genetic organization.............................................. 14

C. Morphological features............................................... 16

D. Metabolism......................................................... 17

E. Virulence Factors.............................................. 18

III. Transcriptional control in bacteria......................................... 21

A. Organization of bacterial promoters................................ 21

B. Organization of borrelial promoters................................. 23

C. Processing of bacterial mRNA........................................ 25

IV. Bacterial responses to stress and environmental signals..................... 27

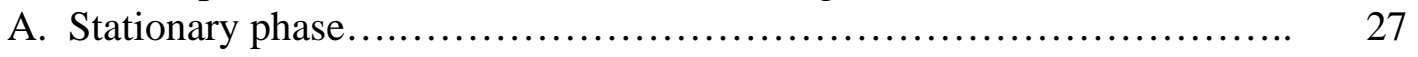

B. Heat shock response........................................... 28

C. Borrelial heat shock response..................................... 29

D. Oxidative stress response............................................ 30

E. Borrelial oxidative stress response.................................... 33

F. Metalloregulation of oxidative stress.............................. 35

V. SecA-dependent protein secretion..................................... 40

A. Overview of secretion mechanisms.................................. 40

B. Transcriptional organization and regulation of SecA................... 42

C. Secretory genes in B. burgdorferi...................................... 43

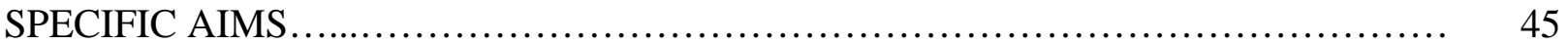




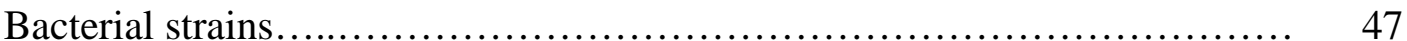

Media and growth conditions......................................... 47

RNA isolation......................................................... 50

Northern blot analysis............................................... 50

Reverse transcriptase polymerase chain reaction (RT-PCR)................. 53

Primer extension (PE) analysis......................................... 53

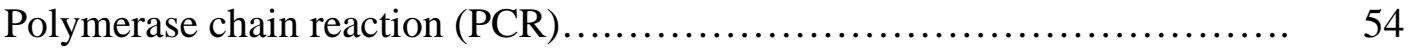

DNA sequencing...................................................... 54

Ribonuclease protection assay (RPA).................................. 55

Secondary structure analysis........................................... 57

Preparation of cell-free extract (CFE) ................................. 57

Sodium dodecyl sulfate- polyacrylamide gel electrophoresis

(SDS-PAGE).................................................. 58

Non-denaturing PAGE and SOD activity staining......................... 58

Spectrophotometric analysis of SOD activity .............................. 59

Two-dimensional gel electrophoresis................................... 59

In-gel tryptic digestion of proteins...................................... 61

Computer imaging..................................................... 62

Statistical analysis................................................... 62

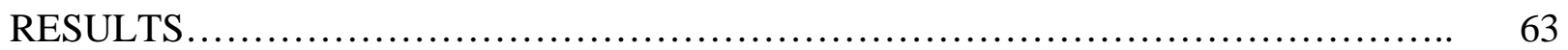

Chapter 1 - Transcriptional analysis of sod in B. burgdorferi.................. 63

Detection of secA-sod transcript................................. 63

Determination of $\sec A$ and $\operatorname{sod}$ promoter........................... 66

Prediction of secondary structure of secA and sod mRNA transcripts.................................................... 79

Chapter 2 - Identifying a possible role for iron in B. burgdorferi............. 82

Identification of Fur-binding boxes.............................. 82

Development of iron-limiting culture conditions.................... 82

Chapter 3 - Expression of secA-sod as a function of metal availability........ 101

Development of ribonuclease protection assay...................... 101

Detection of secA-sod mRNA transcript in different phases of growth............................................ 104

Detection of secA-sod mRNA transcript as a function of metal availability....................................... 107

Chapter 4 - Protein profiling of B. burgdorferi Sh-2-82..................... 114 
Demonstration of SOD activity.............................. 114

Effect of metal availability on protein profiles by SDS-PAGE..... 117

Protein profiling by 2D gel electrophoresis.................... 120

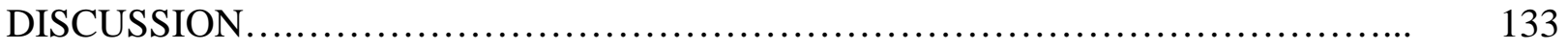

LITERATURE CITED...................................................... 142

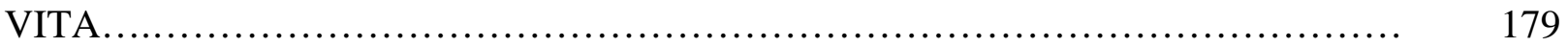




\section{LIST OF TABLES}

Table 1. $\quad$ Formulations of Barbour-Stoenner-Kelly (BSK) media................ 48

Table 2. Oligonucleotide hybridization probe or primer sequences............. 52

Table 3. Biotinylated oligonucletide probes used in ribonuclease protection assays........................... 56

Table 4. Summary of growth patterns of Sh-2-82 in metal-restricted media..... 110

Table 5. $\quad$ Protein identification by MALDI-TOF............................... 124 


\section{LIST OF FIGURES}

Figure 1. Northern blot analysis of Sh-2-82 mRNA......................... 65

Figure 2. $\quad$ RT-PCR analysis of the sod transcript............................. 68

Figure 3. Approximate locations of primers used in primer extension analyses...... $\quad 70$

Figure 4. $\quad$ Lack of $\sigma^{70}$-like promoter region upstream of B. burgdorferi sod.......... 72

Figure 5. $\quad$ PCR using SecA reverse primer and P16F forward primer ............... 75

Figure 6. $\quad$ Putative promoter region of B. burgdorferi secA-sod operon............... 78

Figure 7. $\quad$ Predicted secondary structure of mRNA transcripts using Mfold analysis....................................... 81

Figure 8. $\quad$ Identification of Fur-binding boxes................................. 84

Figure 9. Growth of Sh-2-82 in BSK-II medium in the presence of iron chelators.........................................

Figure 10. Growth of Sh-2-82 in chelexed BSK-II medium in the presence of ferrous ammonium sulfate...

Figure 11. Growth of Sh-2-82 in chelexed BSK-II medium in the presence of ferric nitrate...

Figure 12. Growth of Sh-2-82 in dipyridyl- treated BSK-II medium in the

Figure 13. Growth of Sh-2-82 in BSK-Excyte medium

Figure 14. Effect of manganese on the growth of Sh-2-82.

Figure 15. Development of RPA

Figure 16. Expression of $\sec A$-sod transcript as a function of growth phase

Figure 17. Growth of Sh-2-82 in metal-defined media

Figure 18. Expression of $\sec A$-sod transcript as a function of metal availability........

Figure 19. SOD activity B. burgdorferi Sh-2-82 in response to 
Figure 20. Effect of metal availability on the protein profile of B. burgdorferi Sh-2-82

Figure 21. Protein profiles of Sh-2-82 under different metal conditions using NEPHGE.........................................

Figure 22. Differential protein expression of Sh-2-82 in response to ironrestriction and manganese enrichment............................

Figure 23. Protein profiles of Sh-2-82 under different metal conditions using immobilized $\mathrm{pH}$ gradient gels using SDS-PAGE. ...

Figure 24. Differential protein expression of Sh-2-82 in response to iron-restriction and manganese-enrichment

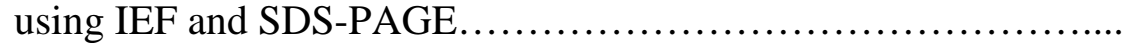




\section{LIST OF ABBREVIATIONS}

\begin{tabular}{|c|c|}
\hline apo-Tf & apo-transferrin \\
\hline ATP & adenosine triphosphate \\
\hline bp & base pair \\
\hline BSA & bovine serum albumin \\
\hline BSK-II & Barbour-Stoenner-Kelly medium \\
\hline BSK-II-Clx & BSK-II treated with Chelex-100 resin \\
\hline BSK-E & BSK medium plus Excyte VLE \\
\hline BSK-E-Clx & BSK-E medium treated with Chelex-100 resin \\
\hline CAT & chloramphenicol acetyltransferase \\
\hline CFE & cell-free extract \\
\hline $\mathrm{CO}_{2}$ & carbon dioxide \\
\hline CuZnSOD & copper-zinc SOD \\
\hline DEPC & diethylpyrocarbonate \\
\hline Dip & dipyridyl, iron chelator \\
\hline Dsf & deferoxamine mesylate, iron chelator \\
\hline ECM & extracellular matrix \\
\hline EDTA & ethylenediamine tetraacetic acid \\
\hline $\mathrm{Fe}$ & iron \\
\hline $\mathrm{Fe}^{2+}$ & ferrous iron \\
\hline $\mathrm{Fe}^{3+}$ & ferric iron \\
\hline $\mathrm{FeCl}_{2}$ & ferric chloride \\
\hline $\mathrm{Fe}\left(\mathrm{NH}_{4}\right)_{3}\left(\mathrm{SO}_{4}\right)_{3}$ & ferrous ammonium sulfate \\
\hline $\mathrm{FeNO}_{3}$ & ferric nitrate \\
\hline FeSOD & iron SOD \\
\hline Fur & ferric uptake regulator \\
\hline GSP & general secretory pathway \\
\hline $\mathrm{H}_{2} \mathrm{O}_{2}$ & hydrogen peroxide \\
\hline HEPES & N-2-hydroxyethylpiperazine-N'-2-ethanesulfonic acid \\
\hline Holo-Tf & holo-transferrin \\
\hline Hsp & heat shock protein \\
\hline IEF & isoelectric focusing \\
\hline IL-1 & interleukin-1 \\
\hline IL-6 & interleukin-6 \\
\hline IL-8 & interleukin-8 \\
\hline IPG & immobilized $\mathrm{pH}$ gradient \\
\hline $\mathrm{kb}$ & kilobase \\
\hline $\mathrm{kDa}$ & kilodalton \\
\hline LB & Lyme borreliosis \\
\hline LD & Lyme disease \\
\hline LYMErix & LD vaccine \\
\hline MALDI-TOF & matrix-assisted laser desorption-time of flight \\
\hline $\mathrm{MgCl}_{2}$ & magnesium chloride \\
\hline $\mathrm{Mn}$ & manganese \\
\hline $\mathrm{MnCl}_{2}$ & manganese chloride \\
\hline
\end{tabular}




$\begin{array}{ll}\text { MnSOD } & \text { manganese SOD } \\ \text { mRNA } & \text { messenger RNA } \\ \text { NaCl } & \text { sodium chloride } \\ \text { NaCN } & \text { sodium cyanide } \\ \text { NAG } & \text { N-acetylglucosamine } \\ \text { NBT } & \text { nitroblue tetrazolium dye } \\ \text { NEPHGE } & \text { non-equilibrium pH gel electrophoresis } \\ \text { NF- } \kappa & \text { nuclear factor-kappa beta } \\ \text { NO } & \text { nitric oxide } \\ \text { NtrA } & \text { sigma factor-54 } \\ \text { OH } & \text { hydroxyl radical } \\ \mathrm{O}_{2} & \text { oxygen } \\ \mathrm{O}_{2}- & \text { superoxide radical } \\ \text { ORF } & \text { open reading frame } \\ \text { Osp } & \text { outer surface protein } \\ \text { PA } & \text { plasminogen activator } \\ \text { PAGE } & \text { polyacrylamide gel electrophoresis } \\ \text { PAS } & \text { plasminogen activation system } \\ \text { PCR } & \text { polymerase chain reaction } \\ \text { PE } & \text { primer extension } \\ \text { PLG } & \text { plasminogen } \\ \text { RBS } & \text { ribosome binding site, Shine-Delgarno sequence } \\ \text { Rf } & \text { relative migration } \\ \text { RNAP } & \text { RNA polymerase } \\ \text { RPA } & \text { ribonuclease protection assay } \\ \text { RpoD } & \text { sigma factor-70 } \\ \text { RpoS } & \text { sigma factor-S } \\ \text { rRNA } & \text { ribosomal RNA } \\ \text { RT } & \text { reverse transcriptase } \\ \sigma & \text { sigma factor } \\ \text { SDS } & \text { sodium dodecyl sulfate } \\ \text { SOD } & \text { superoxide dismutase } \\ \text { SSC } & \text { sodium chloride, sodium citrate solution } \\ \text { Tf } & \text { transferrin } \\ \text { TIGR } & \text { The Institute for Genomic Research } \\ \text { TNF- } \alpha & \text { tumor necrosis factor-alpha } \\ \text { tRNA } & \text { transfer RNA } \\ \text { tPA } & \text { tissue-type PLG activator } \\ \text { uPA } & \text { urokinase-type PLG activator } \\ \text { Zn } & \text { zinc } \\ \text { ZnCl2 } & \text { tinc chloride } \\ \text { 2D } & \\ & \end{array}$




\section{INTRODUCTION}

\section{Lyme Disease}

\section{A. Epidemiology}

Lyme disease (LD) or Lyme borreliosis (LB) is the most commonly reported tickborne infection in North America and Europe. According to the Centers for Disease Control, the overall incidence rate of reported cases of LD in the United States is about 5 per 100,000 population (Orloski et al., 2000). In the U.S., over 90\% of the reported cases occur in the northeastern and north-central states, including Connecticut, Rhode Island, New York, New Jersey, Pennsylvania, Maryland, Massachusetts, and Wisconsin. Connecticut reports the highest incidence rate in the U.S. of 105 per 100,000 population in 1998 (Orloski et al., 2000). Montana is the only state in the U.S. with no reported cases of LD. Unfortunately, very few European countries have made LD a notifiable disease; therefore, incidence rates are only estimations based on clinical studies and prevalence of tick vectors. The World Health Organization (1996) reported that the overall incidence rate is much higher in Europe, excluding England and Ireland, than in the rest of the world. Austria has the highest incidence rate (130 per 100,000 population) and England has the lowest rate (0.6 per 100,000 population).

Although LD has been recognized as an emerging disease, early accounts of LD symptoms have been observed in Europe for about one hundred years (Afzelius, 1910; Garin and Bujadoux, 1922). The multisystemic disorder gained modern attention with an outbreak of “juvenile rheumatoid arthritis” in Lyme, Connecticut in 1975. Steere et al. (1977) 
discovered that a distinctive skin rash associated with a tick bite often preceded the arthritis. Subsequent studies (Burgdorfer et al., 1982) led to the isolation of a spirochete from $I$. dammini (now I. scapularis) ticks (Oliver et al., 1993). The same spirochete was recovered from patients with Lyme arthritis; thereby, establishing the causative agent of LD. The spirochete was named Borrelia burgdorferi in honor of its discoverer, Willy Burgdorfer.

Borrelia burgdorferi sensu lato is divided into ten species: B. burgdorferi sensu stricto, B. bissettii sp. nov., B. japonica, B. lusitaniae, B. garinii, B. tamukii, B. turdi, $\quad$ B. valaisiana, B. afzelii, and B. andersonii (Wang et al., 1999b). Lyme disease in the U.S. results from infection with $B$. burgdorferi sensu stricto; however, isolates of $B$. andersonii and B. bissettii sp nov. have been cultured from ticks and small mammals in the US (Anderson et al., 1989; Postic et al., 1998). European cases of LD are caused by B. afzelii and B. garinii (Hubalek and Halouzka, 1997). Additional borrelial species, B. bissettii, B. lusitaniae, and B. valaisiana have been cultured from ticks, small mammals, or avian reservoirs in Europe and Asia (Kurtenbach et al., 1998; Hubalek and Halouzka, 1997; Nakao et al., 1994). B. japonica, B. tamukii, and B. turdi are found only in Japan (Fukunaga et al., 1996; Nakao et al., 1994). Presently, all reported LD cases have involved the pathogenic species, B. burgdorferi sensu stricto, B. afzelii, and B. garinii.

Although B. burgdorferi isolates are found in many regions of the world, most species are confined to the geographic ranges of specific tick vectors (Bosler et al., 1983; Schrock, 1982). The primary vectors are deer ticks, Ixodes scapularis, in the northeast and upper-midwest states of the U.S. (Steere and Malawista, 1979). Along the west coast of the U.S., the western black-legged tick, I. pacificus, is responsible for transmission of LD spirochetes (Lane and Loye, 1991). I. scapularis is found also throughout the southeastern 
U.S. (Merten and Durden, 2000), but the incidence of LD in the southern states is rare (approximately 0.5 per 100,000 population) (Orloski et al., 2000). Unlike I. scapularis feeding on mammals, I. pacificus ticks feed primarily on the western fence lizard, Sceloporus occidentalis (Lane, 1990; Manweiler et al., 1992). The blood of this lizard is toxic to $B$. burgdorferi (Clover and Lane, 1995; Lane and Quistad, 1998), which results in a lower incidence rate in the western U.S. In Europe, the pathogenic strains, B. garinii and B. afzelii, are transmitted by the sheep tick, I. ricinus, which is the most common tick in northern Europe (Humair and Gern, 2000). I. persulcatus is a common tick found in Eastern Europe and Asia, and is responsible for transmission of LD-spirochetes in Russia (Sato, et al., 1996), China (Li et al., 1998), Japan (Nakao et al., 1994), and Korea (Park et al.,1993).

In the last decade, there has been an increase of many tick-borne diseases (Walker, 1998; Dawson et al., 1996; Dumler and Bakken, 1995; Edlow, 1999). Tick populations have increased along with reforestation and increased deer populations in developed countries (Barbour, 1998). The Ixodes ticks may transmit other zoonotic organisms such as Babesia, causative agent of babesiosis (Mather et al., 1996), and Ehrlichia equi and E. phagocytophilia, which are associated with human granulocytic ehrlichiosis (Walker and Dumler, 1996). Endemic areas of LD overlap with areas of high incidence of human granulocytic ehrlichiosis and babesiosis (Stafford et al., 1999). In addition, antibodies to multiple tick-borne pathogens have been detected in patients with LD (Levin and Fish, 2000; Magnarelli et al., 1995; Nadelman et al., 1997). There is evidence supporting a LD-like infection in the southern U.S. (Barbour, 1996; Barbour et al., 1996; Masters and Donnell, 1995). However, the agent of this LD-like illness has yet to be cultivated (Barbour et al., 1996). The syndrome has similar symptoms of early LD and is transmitted by the hard tick, 
Amblyomma americanum (Lone Star tick) (Campbell et al., 1995). This syndrome has been referred to as southern tick-associated rash infection (Masters and Donnell, 1995). By analyzing 16S rDNA and flagellin genes, Rich et al. (2001) determined that the Lone Star tick-infecting borreliae are closely related to $B$. theileri, the agent of bovine borreliosis. Additionally, A. americanum also may harbor Francisella tularensis, the etiologic agent of tularemia (Taylor et al., 1991), and E. chaffeensis, which is responsible for human monocytic ehrlichiosis (Dumler and Bakken, 1995).

The pathophysiology of the LD spirochetes in the Ixodid tick has been studied by Burgdorfer et al. (1982, 1983, 1989). As the infected tick takes its blood meal on a mammal, the spirochetal population within the tick midgut multiples and migrates to the tick salivary glands (Burgdorfer et al., 1989). The spirochetes pass into the skin of the host at the site of tick attachment within 48 hours (Piesman et al., 1987). Once the transmission has occurred, one of three outcomes may occur. The spirochetes may be eliminated by the host immune system, may establish local infection, or may disseminate to distant sites such as the heart, nervous system, and joints.

\section{B. Clinical features}

Infection with $B$. burgdorferi may be asymptomatic or have a range of clinical presentations, depending on the length of time after the infection and the organs affected. Without antibiotic treatment, the majority of patients’ progress from an early to a late-stage borreliosis. The early stages of LD may not be recognized until later manifestations of the disease are evident. Clinical presentations of LD can be divided into three stages, termed early localized LD, early disseminated LD, and late (chronic) LD. 
In approximately $60 \%$ of the early-localized LD cases, the clinical feature is erythema migrans (EM), the characteristic bulls-eye skin rash spreading from the site of the tick bite (Burgdorfer et al, 1989). The EM lesion appears between two and 30 days after the tick bite and may reach a diameter of 30 inches $(75 \mathrm{~cm})$ with gradual clearing from the central region. The infected patient may also exhibit flu-like symptoms and regional lymphadenopathy close to the EM lesion. Symptoms such as arthralgia (joint pains), myalgia (muscle pains), headache, and neck stiffness have been reported in early LD patients (Shapiro and Gerber, 2000).

Borrelial lymphocytoma is a very rare form of early-localized LD with the majority of cases occurring in Europe (Sigal, 1997). The lesion appears as an intense bluish-red localized painless patch of skin generally on the earlobe of children. The lymphocytoma is a very dense infiltrate of lymphocytes.

The second stage referred to as early-disseminated LD is caused by the spread of the spirochetes to other tissues via the bloodstream and lymphatics. The dissemination to nervous, musculoskeletal, and cardiac systems may cause a wide variation of clinical symptoms presenting within a few weeks to over a year after the initial infection. Peripheral neuropathy (primarily, bilateral Bell’s palsy) and lymphocytic meningitis are the common neurologic manifestations of early disseminated LD in children (Belman et al., 1993; Eggenberger, 1993; Lesser, 1995; Pachner, 1995). Palsy has been observed also in pregnant women infected with B. burgdorferi (Grandsaerd and Meulenbroeks, 2000; Schaumann et al., 1999). Other manifestations of early disseminated LD occurring in children and adults include migratory and recurrent arthralgia (Sigal, 1997) and carditis with conduction defects (usually partial heart block) (Klein et al., 1991). 
Progression to late LD is uncommon but may occur in patients who were treated inadequately at an earlier stage of disease. The third stage presents several months or years after the initial infection and may involve the joints, skin or nervous system. Chronic Lyme arthritis is rare in Europe but is the most common manifestation of LD in the United States (Steere et al., 1977; Shapiro and Gerber, 2000). Lyme arthritis generally affects large joints with the knee joint being most commonly involved. Acrodermatitis chronica atrophicans is an unusual progressive fibrosing skin lesion, which is a common manifestation of late stage LD in Europe (van Dam et al., 1993; Strle et al., 1996; Ruzic-Sabljic et al., 2000). The skin disorder primarily affects the lower limbs of elderly people, starting with a bluish discoloration of the skin, followed by gradual epidermal atrophy. The condition has been shown to be a result of a continuing active Borrelia infection (Asbrink and Hovmark, 1987). Kindstrand et al. $(1997,2000)$ reported that approximately 50\% of patients with acrodermatitis chronica atrophicans exhibit clinical signs of neuropathy. Lyme encephalopathy is rare; however, such neurologic manifestations of Lyme encephalopathy resemble those of chronic fatigue syndrome or fibromyalgia (Kaplan and Jones-Woodward, 1997). Neurologic symptoms are associated with infections with B. afzelii or B. garinii; therefore, LD patients in Europe are more commonly affected by neuroborreliosis.

\section{Immunopathogenesis}

Infection with B. burgdorferi causes a multi-systemic inflammatory disease. While many of the LD manifestations are caused by active infection by the spirochete, others may result from host immunopathogenic mechanisms. Spirochetes are absent generally at sites of 
inflammation during the late stage of LD indicating that the host immune system is responsible for chronic disease. Immune evasion may occur, with the spirochete sequestered in immunoprivileged sites such as joints, heart, and central nervous system, thus enabling relapses in spite of antibiotic treatment (Pachner et al., 1995; Georgilis et al., 1992; PreacMursic et al., 1989; Weis et al., 1997). In addition, it is possible that dead spirochetes or spirochetal fragments may persist and serve as a source for inflammation (Nanagara et al., 1996; Sigal, 1994).

At the site of tick bite and/or needle inoculation, the host initially responds with nonspecific immune responses such as the recruitment of professional phagocytes. Phagocytosis of B. burgdorferi by neutrophils and macrophages has been studied using immunofluorescence, confocal microscopy, and electron microscopy (Benach et al., 1984; Montgomery et al., 1994; Peterson et al., 1984). Although opsonization of B. burgdorferi significantly increased uptake, macrophages and neutrophils also were able to internalize radiolabeled spirochetes in the absence of opsonins (Benach et al., 1984). Phagocytic uptake of opsonized borreliae was blocked by immune complexes, a monoclonal antibody specific for the Fc-gamma receptor III, and a monomeric immunoglobulin G (Benach et al., 1984). The decrease in the number of available receptors on phagocytic surfaces led to a $90 \%$ decrease in the uptake of radiolabeled spirochetes indicating that the majority of phagocytosis of B. burgdorferi occurs through an Fc receptor-mediated process. Electron micrographs have revealed that phagocytes use coiling and conventional phagocytosis for the uptake of B. burgdorferi (Rittig et al., 1992; Montgomery and Malawista, 1996; Rittig et al., 1998). B. burgdorferi, B. garinii, and B. afzelii favored coiling phagocytosis over conventional phagocytosis or macropinocytosis (Rittig et al., 1992; Rittig et al., 1998). 
Montgomery and Malawista (1996) demonstrated progressive engulfment of B. burgdorferi by macrophages; the spirochetes attached by their ends and became progressively coiled as they moved deeper into the cell. Colocalization of spirochetes within lysosomes was identified by labeling cells with antibodies directed against B. burgdorferi and against lysosomal membrane glycoproteins of the endocytic pathway. Spirochetes were degraded rapidly in the lysosomes within 20 to 40 minutes (Montgomery and Malawista, 1996, Montgomery et al., 1993). Electron microscopic examination of phagocytes with internalized B. burgdorferi revealed leaky lysosomes, invagination of large membranous areas, extra-lysosomal degradation of internalized borreliae, and formation of syncytial cells (Rittig et al., 1994).

Using dark-field microscopy equipped with video monitoring, a novel phagocytic pathway, tube phagocytosis, for uptake of $B$. burgdorferi by neutrophils has been observed (Suhonen et al., 1998). At the point of attachment, the neutrophil formed a tube-like protrusion surrounding the spirochete. Once the spirochete was fully covered, the tube was retracted and the spirochete was drawn into the neutrophil. Additional studies are needed to identify the borrelial ligands and the neutrophil receptors involved, as well as the cooperative interactions between the cytoskeleton and the neutrophil membrane in this phagocytic mechanism.

Opsonized and unopsonized spirochetes induced a respiratory burst of nitric oxide (NO) and superoxide radicals $\left(\mathrm{O}_{2}^{-}\right)$in phagocytes (Benach et al., 1984; Peterson et al., 1984; Modolell et al., 1994). Outer surface lipoproteins of B. burgdorferi activate the NO synthase gene stimulating the production of NO by macrophages (Ma et al., 1994) and glial cells (Tatro et al., 1994). In vitro studies (Ma et al., 1994; Modolell et al., 1994) have shown that 
NO and $\mathrm{O}_{2}{ }^{-}$enhance killing of B. burgdorferi by macrophages. However, B. burgdorferi was shown to resist intracellular NO defense mechanisms in vivo (Seiler et al., 1995). Results of this study suggest that NO is not involved in the control of spirochete infection of tissues or in the development of Lyme arthritis. Together these studies indicated that oxygen $\left(\mathrm{O}_{2}\right)$ independent mechanisms associated with phagocytosis might be responsible for degradation of B. burgdorferi in lysosomes. Extracts of neutrophil primary and secondary granules were assayed for borreliacidal activity (Garcia et al., 1998). Elastase, a primary granule, was the only neutrophilic granule effective in killing B. burgdorferi in vitro. The killing activity was independent of the proteolytic activity associated with this enzyme (Garcia et al., 1998). Furthermore, the release of lysosomal contents from damaged phagocytes into the surrounding environment would potentiate the host inflammatory response.

Numerous studies have confirmed that B. burgdorferi lipoproteins promote inflammation by activating a variety of host cell types, resulting in the upregulation of cytokines and adhesion molecules (Ma and Weis, 1993; Talkington and Nickell, 1999; Ma et al., 1994; Wooten et al., 1996; Sellati et al., 1996; Burns and Furie, 1998; Morrison, et al., 1997; Sellati et al., 1999). The outer surface proteins (Osps) of B. burgdorferi are posttranslationally modified, membrane-associated lipoproteins, which possess a tripalmitoyl-S-glyceryl-cysteine moiety at the amino terminus (Brandt et al., 1990). The ability of a tripalmitoyl-S-glycerol-cysteine peptide to penetrate the plasma membrane, and accumulate in the cytoplasm, the nuclear membrane, and the nucleus of macrophages (Uhl et al., 1991) suggests that this lipid moiety may catalyze the formation of a signaling molecule (Erdile et al., 1993; Sellati et al., 1996; Wooten et al., 1998). Lipidated OspA was found to

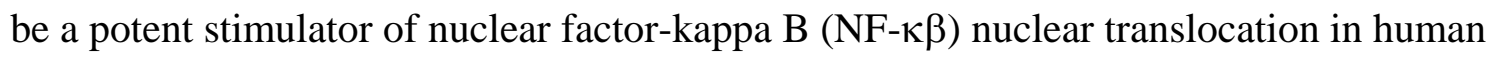


endothelial cells (Wooten et al., 1996). In response to an increase in NF- $\kappa \beta$, synthesis of many proteins essential for localized inflammation was induced. These proteins whose transcription was dependent on NF- $\kappa \beta$ included the cytokine interleukin-6 (IL-6), the chemokine interleukin-8 (IL-8), and the adhesion molecules E-selectin, VCAM-1, and ICAM-1 (Wooten et al., 1996). In a similar study (Isogai et al., 1997), increased levels of interleukin-1 (IL-1 $\alpha$ ), tumor necrosis factor alpha (TNF- $\alpha$ ), and IL-6 were found in various organs the $\mathrm{C} 3 \mathrm{H} / \mathrm{HeN}$ mouse infected with $B$. garinii. This laboratory animal model was developed by Barthold et al. (1993) and has been used extensively in immunization studies. Production of IL-8 was found to be the chemotactic factor for neutrophils required for transendothelial migration (Burns et al., 1997), whereas transendothelial migration of monocytes was dependent on IL-1 (Burns and Furie, 1998). In contrast to other cell types, mast cells were not activated by lipidated, non-lipidated OspA, or the tripalmitoyl-Sglycerol-cysteine moiety; however, incubation with B. burgdorferi resulted in mast cell synthesis and secretion of TNF- $\alpha$ (Talkington and Nickell, 1999). In a subsequent study (Talkington and Nickell, 2001), mast cells exposed in vitro to pathogenic B. burgdorferi cells showed an increased expression of macrophage-inflammatory protein $1 \alpha$ (MIP-1 $\alpha)$, MIP-1 $\beta$, T-cell antigen-3 (TCA3), and IL-6. Mast cell TNF- $\alpha$ secretion was inhibited by incubation with immunoglobulin G1 (IgG1), IgG2 antibodies, and Fc-gamma receptor II and III antibodies, indicating a role for the Fc-gamma receptors in triggering mast cell activation and cytokine release (Talkington and Nickell, 2001). The location of mast cells in the skin places them in close proximity to the inoculation site of borreliae; therefore, the activation of mast cells may intensify the host immune response as well as the inflammatory process. 


\section{Treatment}

For all tick-borne diseases, early diagnosis and treatment improve outcomes. Most infections with $B$. burgdorferi are asymptomatic and self-limiting; however, these infections result in antibody production and limited protection against future challenge. Lyme disease is treated with antibiotics in all stages. Factors to be considered in choosing appropriate antibiotic therapy include efficacy, possibility of co-infections with other tick-borne diseases, and patient status such as age and pregnancy. In early-localized LD, the treatment of choice is either amoxicillin or doxycycline (Hu and Klempner, 2001). Amoxicillin is safe in pediatric and pregnant patients, while doxycycline is used to treat LD when there is a possibility of co-transmitted ehrlichiosis or Rocky Mountain spotted fever. Patients with late stage-LD have been treated effectively with parenteral antibiotics such as cefotaxime or ceftriaxone (Hu and Klempner, 2001).

\section{E. Vaccine}

Many studies in experimental animals demonstrated that humoral immunity protects against infection with B. burgdorferi (Johnson et al., 1986; Barthold and Bockenstedt, 1993; Fikrig et al., 1996; Sadziene et al., 1993; Schaible et al., 1990). These observations generated much interest in the development of a LD vaccine. Both passive transfer of immune sera and antibodies to outer surface protein A (OspA) were effective in preventing infection of severe combined immunodeficiency (Scid) mice (Schaible et al., 1990). Inhibition of infection correlate with the development of high-titered antibodies to a conformational epitope in the C-terminal end of OspA. Anti-OspA antibodies kill B. burgdorferi inside the tick vector, before its transmission to the mammalian target host 
(Fikrig et al., 1992). The design of the LD vaccine is based on the killing of the OspA expressing-spirochete while the spirochete is still within the tick. In unattached and unfed ticks, B. burgdorferi present in the tick midgut expresses OspA (Schwan et al., 1995; Fikrig et al., 1992; Pal et al., 2000). As the tick feeds, a down-regulation of OspA expression coincides with an up-regulation of OspC (Fingerle et al., 1995). Consequently, tick-borne infections in mammals elicit a strong OspC antibody response with a minor (or absent) OspA response. Therefore, the efficacy of the vaccine depends on the rate of spirochetal killing within the tick. In December 1998, the U.S. Food and Drug Administration approved the first LD vaccine (LYMErix, SmithKline Beecham) for use in humans. This vaccine consists of a full-length recombinant OspA (rOspA) from B. burgdorferi strain ZS7 expressed in Escherichia coli as the immunogen (Steere et al., 1998). The monovalent antigen is absorbed to aluminum hydroxide in phosphate buffered saline. The use of a fully lipidated rOspA adsorbed to adjuvant increases antibody titers (Erdile et al., 1993). LYMErix is given in three $30-\mu$ g doses at 0 , one, and 12 months. The overall efficacy of the vaccine in an extensive phase II human trial $(\mathrm{n}=10,936)$ was $76 \%$ after three vaccine injections (Steere et al., 1998). The main adverse reaction reported was tenderness at the injection site and less than $1 \%$ of patients complained of headache, fatigue, or skin rash. As determined by enzyme-linked immunosorbent assay, antibody titers between 700 and 1400 units per milliliter provided $70 \%$ to $95 \%$ protection against LD. In the phase III trials, antibody levels dropped almost 10-fold between the month after the second dose and just prior to the third dose. The third dose at 12 months raised antibody levels higher than that of month two, but these levels declined below protective levels by month 20. Therefore, a booster dose is required to maintain immunity (Steere et al.,1998). The booster schedule has not been 
established and will depend on further studies correlating antibody titers to protection. Presently, LYMErix is approved for use in persons 15 to 70 years of age. The Advisory Committee for Immunization Practices (Centers for Disease Control, 1999) recommends that the vaccine be considered for persons who are at risk for exposure to infected ticks. This population would include people living in endemic areas, hunters, outdoor workers, and veterinarians.

\section{Borrelia burgdorferi}

\section{A. Taxonomy}

Spirochetes represent an ancient grouping of bacteria with a discrete phylogenetic lineage among the eubacteria. The analysis of $16 \mathrm{~S}$ ribosomal RNA (rRNA) gene sequences confirmed that the spirochetes represent a monophyletic phylum among the bacterial phyla (Paster et al., 1991). Spirochetes are presently classified in the Order Spirochetales and are divided into three families, the Leptospiraceae, the Spirochaetaceae, and the Brachyspiraceae. The Spirochaetaceae family is comprised of five genera - nonpathogenic Spirochaeta and Cristispira, and pathogenic Treponema, Spironema, and Borrelia (Paster and Dewhirst, 2000). In molecular typing studies, the genus Borrelia is differentiated from other spirochetal phylogenetic groups by base signature analysis of rrs (Paster et al., 1991). Further analysis of borrelial isolates resulted in identification of more than 20 distinct species within the Borrelia genus (Barbour and Hayes, 1986). Based on differences in their ecological and genetic characteristics, borreliae are categorized as relapsing fever borreliae or LD borreliae. Hundreds of isolates have been cultured from various geographic regions and biological sources to define differences among LD borreliae. A closely related cluster 
containing tick-borne Borrelia species associated with LD has been identified and named $B$. burgdorferi sensu lato (Paster et al., 1991). Borreliae that strictly adhere to the characteristics associated with the species B. burgdorferi are referred to as B. burgdorferi sensu stricto.

\section{B. Genetic organization}

One of the most striking features of B. burgdorferi is its unusual genome, which includes a linear chromosome approximately one megabase in size and numerous linear and circular plasmids. Pulsed-field gel electrophoresis analysis has shown that the chromosome of $B$. burgdorferi migrates as a linear molecule and its migration is unaffected by the introduction of single-stranded nicks (Baril et al., 1989; Ferdows and Barbour, 1989). The linear DNAs have covalently closed hairpin telomeres (Barbour and Garon, 1987). B. burgdorferi has a high extrachromosomal DNA content (approximately 611,000 additional bases) in the form of 12 linear and nine circular plasmids (Barbour and Garon, 1987; Casjens et al., 2000). Plasmid analysis of 13 strains of B. burgdorferi revealed that great variation exists between strains and between in vitro passages of the same strain (Barbour, 1988). Each isolate had four to seven plasmids of various sizes and only two of the 13 had identical profiles. The plasmids have a copy number of approximately one per chromosome (Hinnebusch and Barbour, 1992). Different plasmids often appear to share regions of homologous DNA (Barbour, 1988; Barbour and Garon, 1987). Long-term culture of B. burgdorferi results in the loss of some plasmids (Norris et al., 1992; Purser and Norris, 2000; Schwan et al., 1988; Xu and Johnson, 1995), changes in protein expression profiles (Carroll and Gherardini, 1996; Barbour et al., 1984; Xu et al., 1996) and a loss in the ability of the 
organism to infect laboratory animals (Austin, 1993; Simpson et al., 1990; Xu et al., 1996) suggesting that plasmids encode important proteins involved in virulence. In a recent study (Purser and Norris, 2000), the presence or absence of specific plasmids was examined and correlated with infectivity phenotype. Of the 21 plasmids examined by polymerase chain reaction (PCR) and Southern blot, only one linear plasmid, lp25, was required for infectivity in mice. However, infectivity was enhanced by the presence of lp28-1. As expected, some plasmids were lost after several passages, which suggested that they did not encode essential genes. On the other hand, 13 plasmids were consistently present in all clones. A separate study (Labandeira-Rey and Skare, 2001) confirmed that decreased infectivity was associated with the loss of lp25 and lp28-1. Another unique feature of B. burgdorferi plasmids is the presence of repeated sequences (Carlyon et al., 1998; Casjens et al., 2000; Marconi et al., 1996; Porcella et al.; 1996, Stevenson et al., 1996; Zuckert and Meyer, 1996), which may facilitate homologous recombination among plasmids (Marconi et al., 1994; Palmer et al., 2000; Rosa et al., 1992) and be a source of antigenic variation (Carlyon et al., 1998; Marconi et al., 1993a; Samuels et al., 1993).

The Institute for Genomic Research (TIGR) published the genomic sequence of $B$. burgdorferi strain B31 (Fraser et al., 1997). Sequence data revealed that the genome consists of a linear chromosome of 910,725 base pairs and is very A-T rich with a $\mathrm{G}+\mathrm{C}$ content of only $28.6 \%$. The relatively small genome size correlates with the lack of many biosynthetic genes encoding amino acids, fatty acids, enzyme cofactors, and nucleotides. Homologs of genes encoding an electron transport system also were absent from the sequence analysis. Ninety-three percent of the chromosome represents coding sequences for 853 predicted genes with $59 \%$ of these coding sequences having database matches, $12 \%$ matching hypothetical 
proteins, and 29\% having no database match. The B31 isolate contains 12 linear plasmids and nine circular plasmids accounting for another 610,694 base pairs (Casjens et al., 2000). Sequence analysis of the 21 plasmids found in the B31 isolate predicts 535 intact genes and 167 non-functional genes. The large number of chromosomal and plasmid genes with unknown functions suggests that $B$. burgdorferi is a unique organism, which may regulate its growth, adaptation, and virulence in ways yet to be discovered.

\section{Morphological features}

B. burgdorferi possesses anatomical and physiological characteristics common to all spirochetes (Barbour and Hayes, 1986). Cell length and diameter vary with growth phase and nutritional status. Borreliae are helically shaped with varying degrees of coiling. They possess an outer membrane similar to those in other Gram-negative bacteria except lipopolysaccharide is absent (Takayama et al., 1987). This outer membrane surrounds a helical protoplasmic cylinder, which is surrounded by an inner membrane and contains the cytoplasm. There is a thin layer of peptidoglycan associated with the inner membrane. Unlike most bacterial cell walls, ornithine is incorporated as the diamino acid in the peptidoglycan layer in spirochetes (Klaviter and Johnson, 1969). Motility in spirochetes is achieved by internal organelles called endoflagella that are inserted at each pole and wrap around the protoplasmic cylinder (Holt, 1978; Motaleb et al., 2000). Endoflagella are structurally similar to the external flagella found in other eubacteria. 


\section{Metabolism}

Borrelial metabolism is complex due to the limited metabolic genes found in the genome and the existence of $B$. burgdorferi as a host-associated parasite. Cultivation in laboratory media requires the addition of N-acetylglucosamine (NAG) (Barbour, 1984), which is a major component of both the bacterial cell wall and tick cuticle. This requirement suggests that NAG may be supplied as a carbohydrate source by the tick when borreliae are associated with the invertebrate host. A chitobiase homolog, which may function to convert chitobiose to NAG, has been identified (Fraser et al., 1997). B. burgdorferi can metabolize NAG to fructose-6-phosphate, which can then enter the glycolytic cycle. Genes encoding all of the glycolytic enzymes have been identified in the genome. Glucose, the primary energy source, is metabolized via glycolysis to pyruvate, which is converted to lactate via fermentation. Lactic acid is the primary metabolic end product. This metabolic pathway is consistent with the lack of tricarboxylic acid (TCA) cycle enzymes (Fraser et al., 1997) and the microaerophilic nature of the organism (Austin, 1993). In addition, genes encoding electron transport components are absent in the genome (Fraser et al., 1997); therefore, ATP production occurs exclusively by substrate-level phosphorylation.

Approximately 8\% of the B. burgdorferi genome encodes lipoproteins (Fraser et al., 1997). Lipoproteins are the most abundant proteins in spirochetes with the borrelial outer membranes comprised of 23-50\% lipoproteins. However, the genes encoding fatty acid synthesis are absent (Fraser et al., 1997). Relapsing fever borreliae lack the ability to elongate or $\beta$-oxidize long-chain fatty acids (Livermore et al., 1978). Biochemical studies have shown that the fatty acids in growth media are incorporated unaltered into the cellular lipids (Livermore et al., 1978). Members of the genus Borrelia are cultured routinely in 
Barbour-Stoenner-Kelly (BSK-II) (Barbour, 1984) medium supplemented with bovine serum albumin (BSA) and rabbit serum to provide the required long-chain fatty acids.

\section{E. Virulence factors}

The virulence of any pathogen is determined by the ability of the invading organism to evade or overwhelm the subsequent host immune system. The critical first step in bacterial colonization is adherence while simultaneously evading the host immune response. Once deposited in the dermis of the skin, B. burgdorferi adheres to decorin (Guo et al., 1995), a collagen-associated proteoglycan, via two decorin-binding proteins, DbpA and DbpB (Brown et al., 1999, Guo et al., 1998). However, the spirochete is able to adhere to and penetrate human endothelial cells (Ma et al., 1991; Coleman et al., 1995), thus enabling colonization of tissues. Isaacs (1994) demonstrated that B. burgdorferi adhered to a variety of cell types with attachment mediated by surface-exposed proteoglycans (Leong et al., 1998). As the infection continues, B. burgdorferi travels through the extracellular matrix (ECM) physically interacting with a variety of ECM components to escape the skin and spread to other organs.

In order to invade normal tissues, most bacteria produce proteolytic enzymes to degrade matrix proteins. Pathogenic bacteria without these proteolytic enzymes have devised mechanisms to acquire extracellular proteolytic activity from the host (Coleman and Benach, 1999). The plasminogen activation system (PAS) is a proteolytic system that is primarily responsible for dissolving fibrin clots; however, recently, several bacteria have shown the ability to interact with the host PAS by expressing endogenous or acquiring plasminogen activators (PA) and by binding plasminogen (PLG) through bacterial cell-surface receptors 
(Reddy and Markus, 1972; Broder et al., 1991; Pancholi and Fischetti, 1998; Sodeinde et al., 1988). PLG is converted to its active form, plasmin, as a result of specific proteolytic cleavage by either urokinase-type PLG activator (uPA) or tissue-type PLG activator (tPA). Unbound plasmin is efficiently and specifically inactivated by $\alpha_{2}$-antiplasmin; therefore, it is critical that PLG bound to bacterial surfaces be converted to the active plasmin form and retain its enzymatic activity. High concentrations of PLG are found in blood plasma, thereby, providing B. burgdorferi an opportunity to acquire PLG as the tick takes its blood meal. Consequently, B. burgdorferi has been shown to bind PLG to its surface (Fuchs et al., 1994; Hu et al., 1995; Coleman et al., 1995). Active plasmin is generated in the presence of uPA (Klempner et al., 1995; Coleman et al., 2001) and deposited on the surface of the spirochete. As plasmin-coated B. burgdorferi leave the tick vector and enter into the mammalian host, these spirochetes are able to travel through the ECM by degrading fibronectin (Fuchs et al., 1994; Perides et al., 1996) and other ECM proteins (Coleman et al., 1999). PLG was required for efficient dissemination of B. burgdorferi in ticks and for enhancement of spirochetemia in mice, but was not critical for transmission and infection (Coleman et al., 1997). Likewise, such mechanisms are thought to be responsible for the expansion of the EM rash or multiple EM lesions.

Bacterial phospholipases have been implicated as potential virulence factors which cause a variety of pathophysiological disease states, ranging from massive tissue destruction to interfering with cellular signaling cascades, which modulate the host immune response (Schmiel and Miller, 1999). Phospholipase toxicity generally has been linked to cytolytic activity resulting from hydrolysis of membrane phospholipids Measurement of this lytic activity has been accomplished primarily using hemolysis as a model (Gomez et al., 2001; 
Schmiel and Miller, 1999; Richardson et al.,1986; Stamm and Charon, 1979). Zones of betahemolysis activity were observed around colonies of B. burgdorferi grown on BSK medium containing agarose and horse blood (Williams and Austin, 1992). Both the infective strain Sh-2-82 and non-infective strain B-31 displayed beta-hemolysis after one to two weeks of incubation resulting in complete lysis of the blood plate. Additionally, Guina and Oliver (1997) cloned the small membrane-interactive BlyA protein of B. burgdorferi strain B31, which together with BlyB, promoted hemolytic activity in an E. coli strain encoding this locus. In B. burgdorferi B31, the blyAB locus is located on the cp32 family of conserved circular plasmids and the lp56 linear plasmid (Casjens et al., 1997; 2000). The conserved cp32 plasmid family has been identified as the $\phi \mathrm{BB}-1$ prophage, and linearized cp32 molecules are packaged into bacteriophage particles (Eggers and Samuels, 1999). Recently, Damman et al. (2000) reported that the BlyA and BlyB function as a prophage-encoded holin or holin-like system instead of a hemolysin structure. All known tailed bacteriophages employ a small protein, known as a holin, to form stable, nonspecific pores in the host membrane, allowing muralytic enzymes, endolysins, access to the peptidoglycan to degrade the cell wall (Wang et al., 2000). Damman et al. (2000) proposed that BlyA, as a holin, and BlyB, as an endolysin, play an important role in the lysis of B. burgdorferi cells during the last stage of the $\phi \mathrm{BB}-1$ lytic cycle. Whatever the mechanism of lysis, disruption of borrelial membranes in vivo would result in the release of a significant amount of lipoproteins into the surrounding tissue causing varying degrees of inflammation as described in LD.

Early studies on borrelial Osps revealed a high degree of variability between isolates (Barbour et al., 1984; Marconi et al., 1993a; Marconi et al., 1993c; Jonsson et al., 1992). In addition, the spirochete varies its antigenic composition of OspA and OspC during transit 
from tick to mammalian host (Schwan et al., 1995; Schwan and Piesman, 2000; Obonyo et al., 1999). The major outer surface proteins, OspA and OspB, vary in molecular weight, gene sequence and in reactivity to monoclonal antibodies (Marconi et al., 1993a).

\section{Transcriptional Control in Bacteria}

Bacteria adapt to frequently changing environmental conditions by activating and/or repressing genes encoding metabolic and structural proteins. Consequently, bacteria have developed elaborate mechanisms to regulate transcription as well as translation. Genes can be switched on or off by substitution of the sigma $(\sigma)$ factor(s) associated with the RNA polymerase (RNAP). Regulatory proteins, acting as cis elements, may negative or positively control transcription by binding at the promoter region of the target gene (Raibaud and Schwartz, 1984). In addition, antisense RNA negatively controls transcription of the gene to which it binds. Secondary structure may affect the efficiency of the RNAP binding to the promoter during initiation of transcription. Translation efficiency is greatly affected by messenger RNA (mRNA) stability. The synthesis of bacterial ribosomes is also regulated relative to the growth status of the cell. When bacterial growth slows, ribosomal synthesis is repressed resulting in fewer ribosomes available for translation; thereby, overall translation is repressed.

\section{A. Organization of bacterial promoters}

The DNA sequences involved in regulating transcription generally lie upstream of the protein-coding region of the gene. The sequence that is upstream of the 5 ' end of the gene is referred to as the promoter region. By controlling the ability of RNAP to bind to the 
promoter or, once bound, to transcribe through the entire gene sequence, the bacterium can modulate the amount of mRNA produced.

Transcription initiation depends on the RNAP recognizing the promoter region. The RNA polymerase holoenzyme is a complex enzyme composed of two functional units, the core enzyme and the $\sigma$ factor (Burgess et al., 1969). The core enzyme consists of four polypeptide chains, $\alpha_{2} \beta \beta^{\prime}$, and is responsible for catalyzing the polymerization of ribonucleoside 5'-triphosphates into RNA (Mangel and Chamberlin, 1974; Chamberlin, 1974). The $\sigma$ factor is a single polypeptide chain that is associated weakly with the core enzyme to form the holoenzyme. The major role of the $\sigma$ factor is to direct the core enzyme to specific promoters by recognizing specific sequences within the promoter region (Helmann and Chamberlin, 1988). The presence of multiple $\sigma$ factors has been demonstrated in the prokaryotic kingdom (Doi and Wang, 1986). The various $\sigma$ factors interact with a common core enzyme to form a family of holoenzymes. Bacteria possess one major $\sigma$ factor that is responsible for the vast majority of promoter recognition in transcription initiation. Sigma-70 functions as the major housekeeping $\sigma$ factor in Escherichia coli; $\sigma^{70}$-like factors function likewise in most bacteria (Helmann and Chamberlin, 1988). Minor $\sigma$ factors are synthesized primarily in response to environmental signals or stress conditions (Helmann and Chamberlin, 1988).

Analyses of DNA sequences which $\sigma$ factors recognize revealed two regions that were very similar in a variety of genes (Gribskov and Burgess, 1986; Helmann and Chamberlin, 1988). The common regions encompass six nucleotides each and are located approximately 10 and 35 bases upstream of the transcription start site. These common 
sequences are referred to as the -10 and -35 regions and are separated by approximately 17 bases. Although the sequences at the -10 and -35 positions are not identical in all promoters, they are similar enough to establish consensus sequences. For example, the consensus sequence of the -35 and -10 regions for $\sigma^{70}$ in E. coli is TTGACA-N17-TATAAT (Hawley and McClure, 1983; Harley and Reynolds, 1987). Genes with promoters that differ from the consensus sequences are transcribed less efficiently. Additionally, promoters may be arranged in tandem or overlapping order so that the promoter sequence would be recognized by multiple $\sigma$ factors to allow differential expression of genes in response to environmental signals.

\section{B. Organization of borrelial promoters}

Studies of transcriptional organization and regulation in B. burgdorferi have been hampered by the lack of a genetic exchange system in spirochetes. From the sequenced borrelial genome, three $\sigma$ factor homologs were identified: $r p o D\left(\sigma^{70}\right), n t r A\left(\sigma^{54}\right)$, and $r p o S$ $\left(\sigma^{S}\right)$ (Fraser et al., 1997). Borrelial promoters for genes encoding Osps (Howe et al., 1986; Jianhui et al., 1997), flagellin (Ge et al., 1997), and p39 antigen (Simpson et al., 1994) were identified by their homology to consensus sequences in E. coli. As expected, the predominant $\sigma$ factor appears to be $\sigma^{70}$-like. Analyses of borrelial promoter regions have been accomplished primarily by primer extension (PE) experiments, which yield only information on the structural organization of the region. The transcriptional start site of the osp $A B$ operon in several LD isolates was identified using oligonucleotide primers specific for the 5' region of the ospA coding region (Marconi et al., 1993a). B. garinii and VS461 group isolates possessed an untranslated leader sequence 6 nucleotides longer than that observed in 
B. burgdorferi isolates. Transcriptional analysis of the borrelial membrane protein gene cluster, $b m p A B C D$, indicated that the four genes are organized into two independent transcriptional units with a complex pattern of transcription. The transcription included both alternative monocistronic and polycistronic messages based on PE experiments identifying promoters for $b m p A, b m p C$, and $b m p D$, but not for $b m p B$ (Dobrikova et al., 2001).

Transcriptional start site data obtained via PE experiments in conjunction with transcript size data from Northern analyses suggest how borrelial genes are organized as monocistronic or polycistronic operons.

Functional promoter analyses dependent on a reporter gene have been limited in borreliae. Sohaskey et al. (1997) used chloramphenicol acetyltransferase (CAT) enzyme as a reporter gene in borrelial cells transformed by nonreplicative DNA. The CAT enzyme catalyzes the acetyl coenzyme A-dependent acetylation of chloramphenicol. Transient expression of the cat gene was determined by immunoassays in order to evaluate the promoters of $\operatorname{spA} A, \operatorname{sp} C$, and flaB. Measurement of CAT activity demonstrated that these three promoters were functional with the ospA promoter being about 20-fold more active than the promoterless cat gene. The flaB and ospC promoters also increased CAT activity by seven- and three-fold, respectively (Sohaskey et al., 1997).

Several genetic studies in B. burgdorferi have utilized the gyrB gene encoding the B subunit of DNA gyrase, which is a heterotetramer $\left(\mathrm{A}_{2} \mathrm{~B}_{2}\right)$ comprised of GyrA and GyrB (Samuels et al., 1994; Rosa et al., 1996; Tilly et al., 1997; Knight et al., 2000). DNA gyrase is the only topoisomerase capable of introducing negative supercoiling into closed-circular DNA molecules (Cozzarelli, 1980). The B. burgdorferi gyrA gene is unique in that 939 nucleotides of the C-terminal domain encode another protein, the GyrA C-terminal (Gac) 
protein (Knight and Samuels, 1999). The GyrA C-terminal (Gac) domain protein, found only in the Borrelia genus, is synthesized naturally as an abundant 34-kDa DNA-binding protein with functions similar to the E. coli HU protein (Huisman et al., 1989). The GyrA protein has been demonstrated to be essential in B. burgdorferi (Samuels and Garon, 1993); however, mutations have been introduced successfully into the $g y r B$ allele $\left(g y r B^{r}\right)$ along with a coumermycin $\mathrm{A}_{1}$ resistance $\left(\mathrm{Cou}^{\mathrm{n}}\right)$ marker. Applying this genetic method, Elias et al. (2000) inactivated the $r p o S$ locus by allelic exchange with $g y r B^{r}$ as a selectable marker. To date, coumermycin resistance is the selectable marker of choice for genetic studies in $B$. burgdorferi.

From the genome database, Studholme and Buck (2000) scanned the entire genomic sequence of B. burgdorferi and found that the promoter sequence of rpoS open reading frame (ORF) matches the consensus of $\sigma^{54}$. Transcription by RNAP containing $\sigma^{54}$ is associated with enhancer-dependent regulation in E. coli (Morret and Segovia, 1993). The $B$. burgdorferi genome also encodes a $\sigma^{54}$-dependent activator of the NtrC family; such activators contain an N-terminal "two-component” sensor domain (Sanders et al., 1992). Studholme and Buck (2000) speculate that the reliance of the $r p o S$ promoter on $\sigma^{54}$-RNAP and its cognate activator allows rapid, high-level expression of $r p o S$ to deal with environmental stresses such as entry into stationary phase.

\section{Processing of bacterial mRNA}

Prokaryotic gene expression is controlled primarily at the transcriptional level by virtue of the promoter region. An additional transcriptional control mechanism depends on 
the stability of the transcribed message. The average mRNA is very unstable with a half-life of 40 seconds at $37^{\circ} \mathrm{C}$ (Nilsson et al., 1984). Fortunately, bacterial mRNAs are used immediately as templates for protein synthesis while they are still being transcribed. Bacteria transcribe polygenic messages, which have intergenic spacer regions between ORFs that contain ribosomal binding sites (RBS). The bacterial RBS is known as a Shine-Dalgarno region with a consensus sequence of AGGAGGU, which is complementary to the 3' end of 16S rRNA (Osada et al., 1999). In addition, once a ribosome has sufficiently traversed the RBS, another ribosome may attach and initiate another round of translation. While the mRNA is bound, it is protected from endogenous nucleases. Message stability also may be enhanced by the presence of stem-loop structures at 5' or 3' ends of mRNA.

Limited information is known about the nucleolytic processing of prokaryotic mRNA. The majority of RNA processing reactions occurring within the bacterial cell involves the cleavages necessary to separate the polycistronic messages of the stable RNA species, rRNA and transfer RNA (tRNA). The processing or degradation of mRNA is generally associated with mRNA turnover. Evidence is building that additional mRNA processing is likely to occur with the presence of RNase III found in many sequenced bacterial genomes such as $E$. coli K-12 (Blattner et al., 1997) and B. burgdorferi (Fraser et al., 1997). Unlike RNases P, D, E, and F, RNase III does not have a RNA component and is not responsible for cleavages in processing rRNA or tRNA transcripts (Gegenheimer and Apirion, 1981). Recognition by RNase III is based on the presence of double-stranded-RNA with no apparent consensus sequence. The enzyme cleaves double-stranded-RNA by creating closely spaced singlestrand breaks. Therefore, secondary structures such as stem-loops in promoter regions and/or intergenic spacer regions could be resolved by the action of RNase III. 


\section{Bacterial Responses to Stress and Environmental Signals}

\section{A. Stationary phase}

Many physiological changes occur in a microbe during the transition from exponential growth to stationary phase. Normally, stationary phase is induced as nutrient supplies are exhausted resulting in the cessation of growth. Consequently, microbes have evolved diverse strategies to exploit available nutrient resources and to cope with corresponding environmental stresses to ensure their survival. The molecular processes associated with entry into stationary phase are referred to as the general stress response (Lange and Hengge-Aronis, 1991). Contributing factors include starvation, high osmolarity, temperature fluctuations, and acidic pH (Kolter, 1992; Tao et al., 1999; Suh et al., 1999; Aldsworth et al., 1999; Wise et al., 1996; Svensater et al., 2000). These adverse conditions result in the accumulation of RpoS (Lange and Hengge-Aronis, 1991). RpoS acts as a $\sigma$ subunit of RNA polymerase and binds similar promoter sequences recognized by $\sigma^{70}$ (RpoD). Several research groups working independently on bacterial responses to UVdamage (Tuveson and Jonas, 1979), peroxide stress (Loewen and Triggs, 1984), DNA repair, and starvation identified rpoS (Lange and Hengee-Aronis, 1991). Mutants in rpoS exhibit sensitivity to multiple stresses and die off rapidly in stationary phase (Suh et al., 1999; Kolter, 1992).

A homolog of the rpoS gene was identified in the Borrelia genome (Fraser et al., 1997). The gene assignment was based on $34 \%$ identity (58\% similarity) to Pseudomonas 
aeruginosa rpoS. Recently, inactivation of the rpoS gene in B. burgdorferi and the characterization of an rpoS mutant were described (Elias et al., 2000). The mutant was constructed by inactivating the $r p o S$ locus in the chromosome by allelic exchange with $g y r B^{r}$, a mutated form of the B subunit of DNA gyrase, as a selectable marker. Elias et al. (2000) demonstrated that RpoS facilitates the upregulation of genes involved in entry into stationary phase. The mutant exhibited increased sensitivity to osmotic stress, which is consistent with the finding that wild-type $B$. burgdorferi had a growth phase-dependent resistance to $1 \mathrm{~N}$ $\mathrm{NaCl}$. Using two-dimensional nonequilibrium $\mathrm{pH}$ gradient electrophoresis (2D-NEPHGE), 11 proteins were expressed differentially in the RpoS mutant in comparison to the wild-type strain. Further studies into the rpoS-regulated genes should help elucidate the changes in borrelial gene expression in response to changing environmental conditions.

\section{B. Heat shock response}

Bacteria respond to increased temperature by up-regulating a group of proteins referred to as heat shock proteins (Hsps). These Hsps were identified originally in cells subjected to elevated temperatures; however, Hsps function as molecular chaperones under normal as well as stress conditions. Under normal temperature conditions, Hsps assist in the folding of nascent proteins into their native conformations (Gething and Sambrook, 1992). Unfolded polypeptide chains are protected in the cytosol by being bound within the cavity of the Hsp. As the temperature is elevated, Hsp synthesis increases 10-15 fold within five minutes (Herendeen et al., 1979). In E. coli, this rapid induction occurs primarily by an increase in $\sigma^{32}$ (encoded by rpoH) (Landick et al., 1984; Grossman et al., 1984). The temperature increase causes melting of the rpoH mRNA secondary structure and transient 
stabilization of $\sigma^{32}$ (Kanemori et al., 1994). As a consequence, $\sigma^{32}$ is sequestered away from its association with the Hsps, DnaK, DnaJ, and GrpE. In this way, $\sigma^{32}$ may bind promoters to increase transcription of additional Hsps (Gamer et al., 1992). The -10 and -35 consensus sequences of $\sigma^{32}$, CTTGAA-N(13-17)-CCCCAT (Cowing et al., 1985), differ significantly from that recognized by $\sigma^{70}$. The overall function of the $\sigma^{32}$ regulon has been studied extensively in Gram-negative bacteria by analysis of $r p o H$ mutants. At least 30 genes are regulated by the $\sigma^{32}$ regulon in E. coli (Missiakas et al., 1996).

\section{Borrelial heat shock response}

Studies in B. burgdorferi have demonstrated that the shift in temperature from $20^{\circ} \mathrm{C}$ to $37^{\circ} \mathrm{C}$, similar to the transition from the tick vector to the mammalian host, induces a heat shock response (Carreiro et al., 1990; Cluss and Boothby, 1990; Stamm et al., 1991; Smith, 1998). Homologs of dnaK, dnaJ, and groE have been cloned (Tilly et al., 1993) and identified in the genome of B. burgdorferi (Fraser et al., 1997). Pulse-chase experiments followed by sodium dodecyl sulfate-polyacrylamide gel electrophoresis (SDS-PAGE) revealed that a $75-\mathrm{kDa}$ band, which is similar to the molecular weight of $E$. coli DnaK, was the predominant protein observed during a temperature increase in B. burgdorferi (Cluss and Boothby, 1990). The $75-\mathrm{kDa}$ protein increased from $1 \%$ to approximately $18 \%$ of the total cell protein as the temperature shifted from $28^{\circ}$ to $40^{\circ} \mathrm{C}$. A 60 -kDa polypeptide, presumably GroEL, only increased slightly at the higher temperature. In another study, a total of 12 Hsps were identified as being up-regulated during a temperature increase (Carreiro et al., 1990). The non-infective strain B-31 of B. burgdorferi exhibited an induction of at least seven Hsps 
with a concurrent repression of seven proteins during a shift from $33^{\circ} \mathrm{C}$ to $40^{\circ} \mathrm{C}$; whereas, the infective strain, Sh-2-82, exhibited a different protein profile with an induction of six proteins with different molecular masses than the non-infective strain (Smith, 1998). Immunoblot analysis revealed a 72-kDa protein band reactive with antibodies to both E. coli DnaK and Mycobacterium tuberculosis Hsp71 (Cluss and Boothby, 1990). Likewise, two additional protein bands, 66-kDa and 60-kDa, reacted with antibodies against the $M$. tuberculosis 65-kDa homolog of E. coli GroEL. Antisera from LD patients reacted with the GroEL homologs, but not with the DnaK homolog. Complementation studies (Tilly et al., 1993) demonstrated that the B. burgdorferi grpE and dnaJ homologs complemented E. coli grpE and dnaJ mutants; whereas, the borrelial dnaK gene failed to complement dnaK mutants of E. coli.

\section{Oxidative stress response}

A bacterium undergoes oxidative stress in the presence of excess reactive $\mathrm{O}_{2}$ intermediates (ROIs) in the cell. These intermediates are: $\mathrm{O}_{2}{ }^{-}$, hydroxyl radical ( $\mathrm{HO}$ ), and hydrogen peroxide $\left(\mathrm{H}_{2} \mathrm{O}_{2}\right)$. In aerobically growing E. coli and Salmonella typhimurium, the incomplete reduction of $\mathrm{O}_{2}$ to $\mathrm{H}_{2} \mathrm{O}$ during respiration is the main source of endogenous $\mathrm{O}_{2}{ }^{-}$ and $\mathrm{H}_{2} \mathrm{O}_{2}$ (Farr and Kogoma, 1991). Environmental exposure to radiation, light, metals or redox agents such as paraquat, plumbagin and menadione also cause increased levels of ROIs (Farr et al., 1985). Stimulated macrophages release ROIs during the respiratory burst when foreign bacterial antigens are phagocytosed. Evidence of $\mathrm{O}_{2}$ toxicity includes damage to DNA, RNA, proteins, and lipids (Farr and Kogoma, 1991). 
Aerobic and many aerotolerant organisms have evolved complex enzymatic defense and repair systems to thwart the damaging effects of ROIs. Many of the proteins with their corresponding genes involved in oxidative defense have been identified. Superoxide dismutase (SOD) is widespread in bacteria and is responsible for eliminating $\mathrm{O}_{2}^{-}$by conversion to $\mathrm{H}_{2} \mathrm{O}_{2}$ and $\mathrm{H}_{2} \mathrm{O}$ (Fridovich, 1986). Two forms of catalase, HPI (katG) and HPII (katE), protect against peroxide toxicity by catalyzing the dismutation of $\mathrm{H}_{2} \mathrm{O}_{2}$ to $\mathrm{H}_{2} \mathrm{O}$ and $\mathrm{O}_{2}$ (Loewen et al., 1991). Glutathione reductase reduces disulfide bridges caused by oxidative stress in proteins; similarly, alkyl hydroperoxide ( $a h p)$ reductase acts to reduce hydroperoxides produced in the cell (Storz et al., 1990a). In addition to the oxidative defense enzymes that act directly on the oxidant, repair enzymes are also induced by oxidative stress. In E. coli, apurinic/apyridinic endonuclease activity has been shown to remove replication blocks from the carboxy termini of oxidized DNA (Farr et al., 1985). The protein OxyR also induces the expression of the nonspecific DNA binding protein Dps, which may sequester the DNA within a lattice formation (Wolf et al., 1999). Other enzymes that have been associated with repairing oxidative damage are encoded by $u v r A B$ (Lin and Sancar, 1989), recBC (Imlay and Linn, 1986), and polA (Ananthaswamy and Eisenstark, 1977).

Regulation of the oxidative stress response in prokaryotes involves a complex integration of genes in several regulons. Much of the understanding of the complexities of oxidative defense mechanisms comes from studies in Gram-negative bacteria. In E. coli and S. typhimurium, an excess of $\mathrm{H}_{2} \mathrm{O}_{2}$ induces the expression of approximately 30 proteins (Farr and Kogoma, 1991). HPI catalase (KatG), glutathione reductase, and alkyl hydroperoxide reductase are proteins under the regulation of the OxyR regulon. Upon oxidation, OxyR undergoes a conformation change at a key cysteine residue (Storz et al., 1990a) and binds to 
promoter regions resulting in transcription of enzymes associated with dissipating the effects of $\mathrm{H}_{2} \mathrm{O}_{2}$ (Storz et al., 1990b). On the contrary, HPII catalase (KatE) is not peroxide inducible and is transcribed using RpoS (or KatF) in E. coli (Loewen and Triggs, 1984). KatF functions in an overlapping group of stress-response proteins induced during the transition to stationary phase. Mutations in katF results in increased sensitivity to $\mathrm{H}_{2} \mathrm{O}_{2}$ (Storz and Toledano, 1994).

Elevated levels of $\mathrm{O}_{2}{ }^{-}$induce the two-component system, SoxRS (Nunoshiba et al., 1992). SoxR, the sensor, becomes activated in the presence of $\mathrm{O}_{2}^{-}$thereby causing transcription of soxS. SoxS, the responder, activates transcription of genes such as manganese-containing SOD (MnSOD), the DNA repair enzyme endonuclease IV, and glucose-6-phosphate dehydrogenase, which increases the reducing power of the cell. The $\mathrm{O}_{2}{ }^{-}$ radical is eliminated by SODs, which contain a metallic catalytic center that facilitates electron transfer (Duke, 1978; Fridovich, 1997). SODs are classified according to the metal cofactor and the associated metal properties. Although MnSODs and iron-containing SODs (FeSODs) share sequence homology and are found in prokaryotes, they differ in expression and physiological function (Kirby et al., 1980). The third class of SOD proteins, the copper, zinc-containing SODs (CuZn-SODs), originally thought to be only in eukaryotes, are found in a number of bacteria (Kroll et al., 1995; Benov and Fridovich, 1994; Groote et al., 1997; SanMateo et al., 1998a; Michiels et al., 1994). In E. coli, MnSOD is encoded by sodA and is induced upon exposure to $\mathrm{O}_{2}^{-}$; whereas FeSOD, encoded by $\operatorname{sodB}$, is expressed constitutively (Kirby et al., 1980; Yost and Fridovich, 1973). Localization studies in E. coli have shown that MnSOD is associated with DNA in the cytoplasm to protect against $\mathrm{O}_{2}{ }^{-}$dependent DNA damage (Britton and Fridovich, 1977). FeSOD is also found in the cytoplasm and protects 
cytoplasmic enzymes. CuZn-SOD, encoded by sodC, has been localized to the periplasmic space, suggesting that it functions to protect against extracellular $\mathrm{O}_{2}^{-}$(Kroll et al., 1995).

The metal cofactor of SODs is determined generally by assaying the sensitivity of enzymatic activity to chemical inhibitors (Beauchamp and Fridovich, 1971; Beyer and Fridovich, 1987; Kirby et al., 1980). Typically, FeSODs and CuZn-SODs are sensitive to millimolar concentrations of $\mathrm{H}_{2} \mathrm{O}_{2}$, while MnSODs are more resistant. Cyanide inhibits the CuZn-SOD, but not MnSOD or FeSOD. CuZn-SOD is more resistant to sodium azide than are MnSOD and FeSOD. Results from these relatively simple assays are complicated by evidence of cambialistic SODs, in which either iron (Fe) or manganese (Mn) can function as the metal cofactor in certain bacteria (Chen and Gregory, 1991; Meier et al., 1982;

Gabbianelli et al., 1995). Additionally, some bacteria may possess a SOD with significant sequence homology to MnSOD but is inhibited by $\mathrm{H}_{2} \mathrm{O}_{2}$, thereby resembling a FeSOD (Kusunose et al., 1976; SanMateo et al., 1998b; Zhang et al., 1991).

\section{E. Borrelial oxidative stress response}

An initial study of the distribution of SOD, catalase, and peroxidase activities in spirochetes revealed differences in their enzymatic defenses against $\mathrm{O}_{2}$ toxicity (Austin et al., 1981). Spirochaeta aurantia, Borrelia hermsi, and Leptospira biflexa possessed FeSOD activity, which was inhibited by $\mathrm{H}_{2} \mathrm{O}_{2}$. The first report of SOD activity in infective and noninfective strains of $B$. burgdorferi indicated the enzyme contained Fe (Whitehouse et al., 1997). SOD activity in cellular extracts was assayed using the xanthine oxidase/ cytochrome c method (McCord and Fridovich, 1969). The borrelial SOD activity appeared to be produced constitutively and was not inducible by increased $\mathrm{O}_{2}$ concentrations. In addition, 
no peroxidase or catalase activity was detected (Whitehouse et al., 1997). Likewise, the genomic sequence data did not indicate the presence of genes encoding peroxidase, catalase or homologs of SoxRS or OxyR regulons (Fraser et al., 1997).

Whitehouse et al. (1997) cloned a sod gene from B. burgdorferi strain Sh-2-82 genomic DNA using polymerase chain reaction (PCR) with degenerate oligonucleotide primers corresponding to conserved regions of both MnSOD and FeSOD. The resulting PCR product was used as a probe in Southern blots of BglII-digested Sh-2-82 genomic DNA. A 1.9-kb fragment was isolated and cloned into pUC18; this new recombinant plasmid was designated pCW100. When the borrelial sod was expressed in an E. coli sodAsodB mutant, the recombinant protein was identical to the native borrelial SOD in terms of electrophoretic mobility and sensitivity to $\mathrm{H}_{2} \mathrm{O}_{2}$. The recombinant SOD was enzymatically active and imparted a growth advantage to the E. coli double mutant. DNA sequence analysis revealed a 609-bp ORF encoding a protein of 203 amino acids with a predicted molecular weight of 23,525 daltons. The nucleotide sequence of pCW100 (GenBank assession number AF011226) is identical to the sequence submitted by TIGR (GenBank accession number 2688037) for B. burgdorferi B31 (Fraser et al., 1997), with the exception of two nucleotides. The sod gene is designated as sodA (BB00153) in the B. burgdorferi database; this designation is based on $46 \%$ homology to sodA of Hemophilus influenzae.

B. burgdorferi is grown routinely in a microaerophilic environment to mimic its natural environments in tick and mammalian hosts. Infectivity of B. burgdorferi depends on the number of serial passages in a low $\mathrm{O}_{2}$ environment. Austin (1993) demonstrated that the infective strain Sh-2-82 was able to retain its infective phenotype after 20 passages in $4 \% \mathrm{O}_{2}$ $-5 \% \mathrm{CO}_{2}-91 \% \mathrm{~N}_{2}$. B. burgdorferi cultivated in ambient $\mathrm{O}_{2}$, which is established in the 
head space of tightly capped vessels, lost infectivity between 15 and 20 passages. These results suggest the importance of a constant decreased $\mathrm{O}_{2}$ and increased $\mathrm{CO}_{2}$ environment in preventing the loss of virulence determinants. Differences in protein profiles of infective Sh2-82 and non-infective B-31 strains were compared after a shift in $\mathrm{O}_{2}$ concentration from $4 \%$ to $20 \%$ (Smith, 1998). In $20 \% \mathrm{O}_{2}$, three major proteins with molecular weights of 71-, 66-, and 21-kDa were induced in Sh-2-82. The 71-kDa and 66-kDa proteins potentially represent DnaK and GroEL, respectively. The 21-kDa protein is in the molecular weight range of an Osp, such as OspC, OspE, and OspF. Borrelial Osps are differentially expressed in response to environmental signals. Pulse-labeling experiments with Sh-2-82 exposed to increasing concentrations of $\mathrm{H}_{2} \mathrm{O}_{2}$ resulted in repression of six proteins with estimated molecular weights of 55-, 53-, 37-, 27-, 24-, and 22-kDa (Smith, 1998). Increasing concentrations of paraquat, a generator of endogenous $\mathrm{O}_{2}{ }^{-}$, induced the expression of three proteins at 64-, 27-, and 19-kDa along with the repression of two proteins at 63-kDa and 37-kDa (Smith, 1998).

\section{F. Metalloregulation of oxidative stress}

Many of the transition elements function as essential cofactors in microbial enzymes and are required for growth. A wide range of stress-responsive genes is induced as metal ion concentrations increase from starvation to toxic levels. The deleterious effects that occur under conditions of elevated metal concentration include enzyme inhibition and uncontrolled redox cycling within the cell (Stohs and Bagchi, 1995). Defining the minimum and maximum levels of transition metals required by bacteria remains a goal in studies involving metal homeostasis. However, efforts to establish metal starvation and metal toxicity limits are complicated by changes in metal bioavailability in different media. Excess levels of one 
metal can lead to competition with other metals during cellular transport or competition for active sites in metal-cofactored enzymes. An example of this competition is observed in cambialistic SODs, which the cofactor is dependent on the metal supplied in the medium (Meier et al., 1982).

Aerobic bacteria residing in a neutral or alkaline environment encounter difficulties in acquiring sufficient amounts of Fe and transition metals since these metals are highly insoluble under these conditions. In the presence of $\mathrm{O}_{2}$, free $\mathrm{Fe}$ is limited because ferrous $\left(\mathrm{Fe}^{2+}\right)$ iron is readily oxidized to ferric $\left(\mathrm{Fe}^{3+}\right)$ iron, which quickly forms insoluble hydroxides at neutral pH (Iuchi and Weiner, 1996). To mitigate Fe-limitation, bacteria have evolved several unique Fe-scavenging strategies. A common feature of metal uptake systems is that their expression is induced by metal ion depletion. The synthesis of siderophores and specialized transferrin and/or lactoferrin receptors facilitates Fe uptake (Briat, 1992).

Strict regulation of Fe assimilation prevents an excess of free intracellular Fe that may lead to oxidative stress. Ferrous iron facilitates the conversion of the less reactive $\mathrm{H}_{2} \mathrm{O}_{2}$ to the more reactive $\mathrm{O}_{2}$ species, $\mathrm{OH} \cdot$ and $\mathrm{Fe}^{3+}$, via the Fenton reaction (Imlay et al., 1988). Therefore, maintaining Fe homeostasis requires a balance between Fe transport into the cell and Fe accumulation within the cell.

Recent studies in Fe availability and utilization by spirochetes have revealed unique Fe requirements contrary to that of eubacteria (Posey et al., 1999; Jovanovic et al., 2000; Posey and Gherardini, 2000). Iron has been considered a growth-limiting factor for microorganisms in most natural environments due to its participation in diverse biological processes, such as cellular respiration and transcriptional regulation (Briat, 1992). Analysis of B. burgdorferi membranes indicated that metalloproteins, such as cytochromes, respiratory 
proteins or tricarboxylic acid enzymes, commonly associated with bacterial cytoplasmic membranes, were lacking (Bledsoe et al., 1994). Likewise, the complete genome sequence of B. burgdorferi confirmed that these genes were missing (Fraser et al., 1997). Only three metalloproteins have been identified in borreliae; these include a SOD, a ferric-uptake regulator protein (Fur), and a putative neutrophil-activating protein (Whitehouse et al., 1997; Fraser et al., 1997). Posey and Gherardini (2000) proposed that B. burgdorferi has undermined the role for Fe by eliminating most of the genes that encode proteins that require Fe as a cofactor. The metalloproteins present in B. burgdorferi may substitute Mn for Fe as the metal requirement. For example, the sod gene in B. burgdorferi exhibits greater sequence homology to that of a MnSOD than to a FeSOD and the fur gene has greater than $50 \%$ homology to the Bacillus subtilis perR gene product, which requires $\mathrm{Mn}$ as a cofactor. Most bacteria require micromolar amounts of Fe to support growth; however, the intracellular Fe concentration in B. burgdorferi was estimated to less than 50 pmol per milligram of protein. Iron concentration in another spirochete, Treponema denticola, was measured also and found to be slightly reduced (3.5 nmol) in comparison to E. coli $(4.2 \mathrm{nmol})$. Relatively few Febinding proteins have been predicted from the genome of Treponema pallidum (Fraser et al., 1998). The predicted neelaredoxin homolog (TP0823) has been characterized as a superoxide reductase utilizing Fe as an active site cofactor (Jovanovic et al., 2000; Lombard et al., 2000). However, further studies are needed to ascertain whether these proteins utilize only Fe or that some other competent metal ion may be substituted as the cofactor. Posey et al. (1999) characterized a unique regulatory system in T. pallidum for a transport-related operon, troPO, in which the associated repressor protein, TroR, was dependent on Mn instead of Fe. Nonetheless, metalloregulation studies in spirochetes are a relatively new area 
of research with potential answers to how microorganisms cope and/or adapt to low metal bioavailability.

Iron uptake is regulated in Gram-negative bacteria by the repressor protein Fur (Bsat and Helmann, 1999; Griggs and Konisky, 1989; Stojiljkovic et al., 1994; Zheng et al., 1999). The Fur protein of $E$. coli consists of two binding domains: an N-terminal DNA-binding domain and a metal-binding C-terminal domain. Spectroscopic analysis has revealed Zn atoms are bound at one site (Althaus et al., 1999), while the second binding site is generally occupied by $\mathrm{Fe}^{2+}$. Manganese ion $\left(\mathrm{Mn}^{2+}\right)$, cobalt $\left(\mathrm{Co}^{2+}\right)$, or other divalent cations may be substituted for the Fe requirement.

Footprinting experiments have identified Fur binding regions within the promoter region of Fe-regulated genes (Frechon and Cam, 1994; Ochsner et al., 1995). Analysis of such regions yielded a Fur-binding consensus sequence or "Fur box," which consisted of a 19-bp palindromic sequence, GATAATGATAATCATTATC. Recently, studies have suggested that Fur recognizes the short hexamer GATAAT with a minimum requirement of three hexamers in any orientation (Escolar et al., 1998).

The Fur protein functions to regulate transcription of Fe-regulated genes in many different bacteria. As Fe accumulates within the cell, $\mathrm{Fe}^{2+}$ binds to the carboxy terminus of the regulatory protein; thereby, the protein acquires structural conformation capable of binding target DNA sequences, such as Fur boxes. Experimental evidence indicates that the mere presence of a Fur box within a promoter region subjects the adjacent genes to Furregulation (Bsat et al., 1998; Calderwood and Meklanos, 1988; vanVliet et al., 1999; Bsat et al., 1999; Leoni et al., 2000). Mutants lacking Fur exhibit defective regulation in numerous cellular responses such as amino acid synthesis (Weisbach and Bsat, 1991), purine nucleotide 
synthesis (He et al., 1993), and toxin production (Tai et al., 1990), suggesting that Fur is a global regulator and not restricted solely to Fe regulation.

The Fur protein acts synergistically with other global regulatory systems to regulate oxidative stress response to $\mathrm{O}_{2}{ }^{-}$in E. coli (Compan and Touati, 1993; Zhen et al., 1999). Febound Fur represses transcription of E. coli sodA and Fe assimilation genes (Niederhoffer et

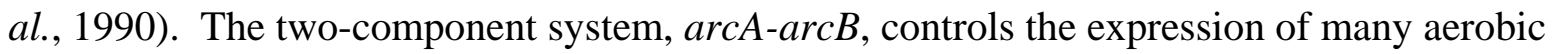
metabolic genes. The aerobic regulation control (ArcA) protein represses sodA during anaerobiosis (Gunsalus and Park, 1994). The DNA-binding protein, integration host factor (IHF), induces a bend in the DNA, which complicates binding of the transcriptional machinery to sodA promoter region (Presutti and Hassan, 1995). The fumarate nitrate reductase (Fnr) protein also binds Fe and is involved in the anaerobic activation or repression of numerous genes including sodA (Gunsalus and Park, 1994).

Peroxide stress elicits a unique response in the Gram-positive bacterium, B. subtilis, which does not involve OxyR. $\mathrm{H}_{2} \mathrm{O}_{2}$ treatment in combination with metal ion limitation increased expression of catalase ( $k a t A)$, alkyl hydroperoxide reductase ( $a h p C F)$, a Dps homolog ( $m r g A)$, and the heme biosynthesis operon (hemAXCDBL) (Chen et al., 1995). The promoters of these $\mathrm{H}_{2} \mathrm{O}_{2}$-induced genes contain a consensus sequence, CTAtNTTAtAATNATTATAAattA (Chen et al., 1995). This 24-bp sequence, referred to as a "Per box," has homology to a Fur box. The completed B. subtilis genome sequence revealed three fur homologs: $y d k L, y q f V$, and $y g a G$ (Bsat et al., 1998). Like fur mutants, the $y d k L$ mutant constitutively expresses genes for siderophore biosynthesis and Fe uptake. The ygaG mutant is resistant to $\mathrm{H}_{2} \mathrm{O}_{2}$ and overexpresses catalase, alkyl hydroperoxide reductase, and the DNA binding protein MrgA. Thus, the ygaG mutant has the perR phenotype. 
Similar to Fur, PerR has a DNA-binding region in the N-terminus with two metal-binding motifs in the C-terminal region. PerR requires a divalent metal ion, Mn and/or Fe, to activate its DNA binding activity (Chen et al., 1993). The peroxide sensing mechanism is unclear. The bound metal ion may play a key role in peroxide sensing, either by contributing to the metal-catalyzed oxidation of the PerR peptide or by a change in the oxidation state of the bound metal ion. In either case, $B$. subtilis peroxide stress response differs from the OxyR regulation observed in most Gram-negative bacteria. Although no OxyR regulation occurs in the Gram-negative bacterium, Campylobacter jejuni, this organism does possess two Fur homologs, Fur and PerR (vanVliet et al., 1998; 1999). Characterization of the C. jejuni perR mutants exhibited repression of alkyl hydroperoxide reductase and catalase genes similar to that observed in B. subtilis. Recent studies in Staphylococcus aureus (Singh et al., 1999; Xiong et. al., 2000; Horsburgh et al., 2001; Lindsay and Foster, 2001) also have shown transcriptional regulation of oxidative stress genes by PerR. In addition to PerR and Fur, $S$. aureus encodes a third homolog, Zur. Lindsay and Foster (2001) showed that Zur represses expression of an ABC-type transporter in a $\mathrm{Zn}^{2+}$-dependent manner. Future studies on the roles of PerR and Zur will help elucidate the mechanisms of transcriptional regulation that has not been ascribed (or has been ascribed incorrectly) to Fur based on metal binding.

\section{SecA-Dependent Secretion}

\section{A. Overview of secretion mechanisms}

Approximately $20 \%$ of the proteins synthesized by bacteria are destined to become an associated membrane component with a few proteins being secreted extracellularly. The 
process, referred to as protein export or translocation, involves the insertion of proteins into membranes requiring the passage of hydrophilic proteins through a hydrophobic membrane (Pugsley, 1993). The first step in protein export is insertion into the cytoplasmic membrane. Subsequent events are dependent on the structure of the outer surface structures of the bacterial cell (Fernandez and Berenguer, 2000). In Gram-positive cells, proteins may remain in the membrane via a membrane anchor or be fully translocated across the cytoplasmic membrane and released as extracellular proteins (Simonen and Palva, 1993). Gram-negative cells present the translocated protein with an additional outer membrane. Translocated proteins may reside in the periplasm or continue to and/or through the outer membrane.

Most secretory proteins are synthesized initially as a precursor protein containing an $\mathrm{N}$-terminal signal sequence, which functions to direct the precursor protein to the cytoplasmic membrane (Pugsley, 1993). The signal sequence includes a hydrophobic region of at least 11 amino acid residues preceded by one or more positively charged residues in a short hydrophilic region. Although all bacterial signal peptides are similar in structure, signal peptides from distinctly different classes of proteins vary in length and sequence composition. Signal peptides of proteins destined for the periplasm, outer membrane, or extracellular environment are cleaved generally by a signal peptidase during translocation across the cytoplasmic membrane.

Many proteins reach their final destination via the general secretory pathway (GSP) (Pugsley, 1993). This transport system is dependent on the sec gene products, SecA, -B, -D, -E, -F, and $-\mathrm{Y}$. SecB, acting as a molecular chaperone, associates with nascent proteins as they emerge from the ribosomes by directly binding to signal peptides. The protein SecA may carry out this function in the absence of SecB. However, the primary role of SecA is as 
an ATPase (Lill et al., 1989), which functions as a molecular motor to promote protein secretion at translocation sites that consist of SecYE. Located within the N-terminus of SecA is a segment of sequence identical to "Walker A boxes" (Walker et al., 1982) which are normally found only in nucleotide-binding proteins such as ATPases or kinases.

Biochemical studies on the interaction of SecA with the preprotein suggest that SecA binds to a high-affinity ATP binding site within a hydrophobic region of a signal peptide (Akita et al., 1990). Complexed SecA proceeds to translocation sites in the inner membrane resulting in activation of the ATPase activity associated with SecA (Akita et al., 1990; Qi and Bernstein, 1999). SecA undergoes nucleotide-modulated cycles of membrane insertion and de-insertion utilizing the energy of ATP binding to insert into the cytoplasmic membrane and to release the signal sequence domain of the bound preprotein. ATP hydrolysis drives the release of the preprotein from SecA and promotes de-insertion of SecA from the cytoplasmic membrane (Economou et al., 1995). This cycle continues until the preprotein is threaded into the membrane. Immediately following translocation through the cytoplasmic membrane, a signal peptidase (LepB) cleaves the signal sequence of the exported protein (Black et al., 1992; Black, 1993). Continued exportation of proteins to the various outer compartments results from interactions of periplasmic proteins (Pugsley, 1990). Many pathogenic Gramnegative bacteria utilize the GSP to export virulence factors such as toxins and pili into the extracellular environment for interaction with the target host (Pugsley, 1990).

\section{B. Transcriptional organization and regulation of SecA}

The SecA-dependent protein secretion pathway has been speculated to be present in all Eubacteria. Homologs of secA have been identified in many Gram-negative and Gram- 
positive bacteria (Blattner et al., 1997; Fraser et al. 1995; McNicholas et al., 1995); however, no secA homolog has been identified in the completed sequence of Methanococcus jannaschii (Bult et al., 1996). Most information about SecA has been gained through studies in E. coli. The E. coli secA ORF contains 2703-bp encoding a protein of 901 amino acids (10.2-kDa) (Beall and Lutkenhaus, 1987). The secA gene is the second gene in an operon comprised of secM-secA-mutA and is transcribed from a $\sigma^{70}$ promoter (Rajapandi et al., 1991; Sarker et al., 2000). SecA autoregulates itself in E. coli by binding to the operator region of its own mRNA transcript. In this manner, SecA competes with ribosomes and inhibits translation (Salavati and Oliver, 1995). Because SecA cycles in and out of the cytoplasmic membrane, soluble and membrane-associated protein forms have been identified (Chen et al., 1998).

\section{Secretory genes in B. burgdorferi}

In the case of $B$. burgdorferi, an overabundance of lipoproteins in the outer membrane suggests the presence of an efficient secretory mechanism. However, there is limited knowledge of how these borrelial proteins are secreted and processed. A secA homolog in B. burgdorferi was cloned using degenerate oligonucleotide primers homologous to the highly conserved ATP-binding sites (Walker boxes) (Guina et al., 1998). Sequence alignment with other bacterial SecA homologs demonstrated that B. burgdorferi SecA was most similar to E. coli SecA with 43.2\% homology. However, the borrelial SecA showed more evolutionary divergence than any of the other SecA homologs except that of Mycoplasma genitalium. In addition to SecA, the completed genome sequence revealed the

presence of all the genes of the GSP except SecB (Fraser et al., 1997). Although limited data 
exist on secretion mechanisms in spirochetes, the presence of GSP homologs indicates that secretion resembles that of type-II secretion found in E. coli and other Gram-negative bacteria (Haake, 2000). 


\section{SPECIFIC AIMS}

B. burgdorferi has been characterized as a microaerophilic spirochete and is cultivated in BSK-II medium in ambient air. Consumption and utilization of $\mathrm{O}_{2}$ potentially yield ROIs, such as $\mathrm{O}_{2}{ }^{-}, \mathrm{OH}$, and $\mathrm{H}_{2} \mathrm{O}_{2}$. While $B$. burgdorferi requires $\mathrm{O}_{2}$ for growth, it fails to grow in strictly aerobic or strictly anaerobic environments. Previous studies in this laboratory have identified SOD activity in B. burgdorferi (Whitehouse et al., 1997), which is responsible for eliminating $\mathrm{O}_{2}{ }^{-}$. On the basis of physiological inhibition by $\mathrm{H}_{2} \mathrm{O}_{2}$, the enzyme was considered to be an FeSOD (Whitehouse et al., 1997). However, the genomic sequence of the B. burgdorferi sod most closely resembles a MnSOD (Fraser et al., 1997). The parameters determining transcription and regulation of sod expression in B. burgdorferi have not been addressed. SOD activity in other bacteria is regulated at the promoter level by the SoxRS regulon, which there is no homolog in B. burgdorferi. In most bacteria, sod is transcribed as a monocistronic message from its own promoter. The start codon of the $B$. burgdorferi sod lies only 17 bases downstream from the stop codon of secA, which encodes an ATPase involved in protein secretion in many Gram-negative bacteria. The close proximity of sod to secA coding regions suggests that their transcription may be coupled. Furthermore, studies in many bacteria have demonstrated that expression of SOD activity is sensitive to the intracellular iron concentration, which is regulated by the Fur protein.

The principal hypothesis that may be drawn from the observed $\mathrm{O}_{2}$ requirement and potential $\mathrm{O}_{2}$ toxicity is that the expression and regulation of the sod gene contribute to the microaerophilic nature of B. burgdorferi. 
The specific aims of this study are:

1. Characterization of the sod promoter in B. burgdorferi

Experiments were performed to: a) determine the size of mRNA transcript and b) determine the transcription start site.

2. Characterization of the role of Fe and Fur in sod expression in B. burgdorferi Experiments were performed to: a) identify a Fur-binding box in the secA promoter region, b) determine SOD activity in borreliae grown in Fe-enriched or Fe-deficient medium, and c) determine levels of sod mRNA as a function of Fe concentration.

3. Determination of protein profiles in borreliae grown in metal-defined media. Experiments were performed to: a) identify differences in protein expression as a function of Fe and Mn availability, and b) identify specific protein by using 2D- gel electrophoresis coupled to MALDITOF.

As the sole oxidative defense enzyme presently known in Borrelia, SOD expression is invaluable to the survival of this organism in a microaerophilic environment. The information gained from these studies will provide a better understanding of the transcriptional regulation of sod in B. burgdorferi. 


\section{MATERIALS AND METHODS}

Bacterial strains. The Sh-2-82 strain of B. burgdorferi was obtained from W. J. Simpson, Rocky Mountain Laboratories, Hamilton, Montana. This strain had been isolated from Ixodes scapularis ticks and passaged fewer than 10 times in vitro. Infectivity of Sh-282 in neonatal Lewis rats has been described previously (Austin, 1993). A clone of Sh-2-82 obtained by single-cell isolation (Williams and Austin,1992) was used throughout this study. Whitehouse et al. (1997) identified the presence of a sod gene and absence of any catalase or peroxidase genes in this clone. New cultures were initiated from the frozen stock and subculturing was limited to six serial passages. A wildtype K-12 strain, E. coli EMG-2 (ATCC 23716) was obtained from the American Tissue Culture Collection (Rockville, Maryland).

Media and growth conditions. B. burgdorferi Sh-2-82 was cultured routinely in BSK-II medium supplemented with 6\% heat-inactivated rabbit serum (Barbour, 1984). Formulation of BSK-II medium is shown in Table 1. Cultures were incubated statically at $33^{\circ} \mathrm{C}$ under ambient $\mathrm{O}_{2}$ conditions established by filling tightly capped culture vessels threefourths full to minimize head air-space. Initial inocula for all cultures were $1 \times 10^{6}$ cells per milliliter. Spirochetes were enumerated by direct cell counting using dark-field microscopy with a Petroff-Hausser counting chamber. Under normal growth conditions, stationary phase in B. burgdorferi Sh-2-82 was defined as the period of bacterial growth when the cell density reached $10^{8}$ spirochetes per milliliter, which was accomplished generally after 96 hours. 


\section{Table 1. Formulations of Barbour-Stoenner-Kelly (BSK) media}

\begin{tabular}{lll}
\hline Component & BSK-II & BSK-Excyte \\
\hline CMRL 1066 with glutamine & $1 \mathrm{X}$ & $1 \mathrm{X}$ \\
Neopeptone & $0.39 \%$ & $0.50 \%$ \\
Bovine serum albumin, fraction V & $3.92 \%$ & $7.00 \%$ \\
TC Yeastolate & $0.16 \%$ & $0.16 \%$ \\
HEPES & $19.7 \mathrm{mM}$ & $19.7 \mathrm{mM}$ \\
Glucose & $21.7 \mathrm{mM}$ & $21.7 \mathrm{mM}$ \\
Sodium citrate & $1.9 \mathrm{mM}$ & $1.9 \mathrm{mM}$ \\
Sodium pyruvate & $5.7 \mathrm{mM}$ & $11.6 \mathrm{mM}$ \\
N-acetylglucosamine & $1.4 \mathrm{mM}$ & $2.9 \mathrm{mM}$ \\
Sodium bicarbonate & $20.5 \mathrm{mM}$ & $20.5 \mathrm{mM}$ \\
Gelatin & $1.10 \%$ & - \\
Heat-inactivated rabbit serum & $6.00 \%$ & - \\
Excyte-VLE & - & $2.00 \%$ \\
Glycerol & - & $0.80 \%$ \\
3-phosphoglyceric acid & - & $1.9 \mathrm{mM}$ \\
\hline
\end{tabular}

The specific Fe chelators, deferoxamine mesylate (Desferal) and 2,2'-dipyridyl were used to restrict Fe availability in BSK-II medium. Initial Fe-reconstitution studies assayed the possible utilization of Fe via Fe-loaded transferrin (Tf). To further define metal composition, Sh-2-82 was grown in BSK-Excyte (BSK-E) medium (Posey and Gherardini, 2000) with modifications of metal content. The formulation of BSK-E medium is compared with that of BSK-II medium in Table 1. The BSK-E medium formulation lacks rabbit serum, which is a potential source of Fe. Excyte-VLE (Serological Proteins, Kanakee, Illinois) is 
added to BSK-E medium as a source of lipoproteins to replace the serum requirement. Additionally, the gelatin component of BSK-II is substituted with 3-phosphoglyceric acid and glycerol in BSK-E for the synthesis of essential lipids.

Pre-cultures in the different media formulations were necessary to dilute out possible metal (or divalent ion) carry-over contamination from the stock culture grown in BSK-E. Initially, $100 \mathrm{ml}$ of BSK-E medium was inoculated from a frozen stock of Sh-2-82 and incubated statically at $33^{\circ} \mathrm{C}$ for three days. Ten milliliters of this initial culture was washed twice in buffer containing $20 \mathrm{mM}$ HEPES, $100 \mathrm{mM} \mathrm{NaCl}$, and $10 \mathrm{mM}$ EDTA and used to inoculate metal-defined media. Cultures were incubated for five days. Cells were harvested and resuspended into fresh media of varying metal concentrations at a final density of $1 \times 10^{6}$ per ml.

For Fe-restricted conditions, dipyridyl was added to BSK-E medium. Additional metal-limiting conditions were accomplished by treating either BSK-II or BSK-E media with Chelex-100 resin (BioRad, Hercules, California). Using the batch method of chelation, 20 grams of Chelex-100 per liter was added to BSK-II medium and stirred for six hours at room temperature. The resin was removed by filtration and the ion-depleted BSK-II (BSK-II-Clx) was filter-sterilized. Iron compounds, ferric nitrate $\left(\mathrm{FeNO}_{3}\right)$ or ferrous ammonium sulfate $\left[\mathrm{Fe}\left(\mathrm{NH}_{4}\right)_{3}\left(\mathrm{SO}_{4}\right)_{3}\right]$, were added aseptically to the medium at varying test concentrations. Additionally, the BSK-E medium was passed over a Chelex-100 resin column at a flow-rate of five centimeters per minute. The chelated BSK-E medium (BSK-E-Clx) was reconstituted with $600 \mu \mathrm{M}$ magnesium chloride $\left(\mathrm{MgCl}_{2}\right), 10 \mu \mathrm{M}$ manganese chloride $\left(\mathrm{MnCl}_{2}\right), 10 \mu \mathrm{M}$ zinc chloride $\left(\mathrm{ZnCl}_{2}\right)$, and/or $10 \mu \mathrm{M}$ ferric chloride $\left(\mathrm{FeCl}_{3}\right)$. All metallic chemicals were 
purchased from Sigma Chemical Company (Saint Louis, Missouri). Cultures were grown at $33^{\circ} \mathrm{C}$ under ambient conditions to stationary phase.

E. coli EMG-2 strain was grown aerobically in Luria-Bertani medium at $37^{\circ} \mathrm{C}$ in a rotary shaking water bath (200 rpm). Growth was monitored by measuring the optical density (OD) at a wavelength of $600 \mathrm{~nm}$ using a DU-650 UV/Vis spectrophotometer (Beckman-Coulter, Fullerton, California).

RNA isolation. Total RNA was extracted from borrelial cultures using the Ultraspec II RNA isolation system (Biotecx Laboratoies, Houston, Texas) according to manufacturer's instructions. The procedure is based on the use of two denaturing agents, guanidine salts and urea, followed by purification of RNA with a RNA-specific resin. RNA pellets were resuspended in diethylpryrocarbonate (DEPC)-treated $\mathrm{H}_{2} \mathrm{O}$. The $A_{260}$ was measured and multiplied by a factor of $40 \mu \mathrm{g}$ per $\mathrm{ml}$ and the dilution factor to determine the RNA concentration (Borer, 1975). RNA purity was assessed by determining the $A_{260} / A_{280}$ ratio. RNA preparations were stored at $-70^{\circ} \mathrm{C}$ until used. In all RNA procedures, water and buffer solutions were treated with DEPC to inactive RNases. Glassware was also treated with DEPC and then baked at $300^{\circ} \mathrm{C}$ for at least 4 hours.

Northern blot analysis. Borrelial RNA from early-stationary cultures grown in BSK-II medium was isolated as described above. The RNA $(10 \mu \mathrm{g})$ was denatured with glyoxal and dimethyl sulfoxide and electrophoresed in a 1.2\% (w/v) agarose gel in $10 \mathrm{mM}$ sodium phosphate buffer, pH 7.0 (Sambrook et al., 1989) as described previously (Stevenson et al., 1998). The RNA was transferred to nylon membranes by capillary action and cross- 
linked by baking at $65^{\circ} \mathrm{C}$ for 1 hour in a vacuum oven. Oligonucleotide probes were 5 '-endlabeled with $\left[\gamma-{ }^{32} \mathrm{P}\right] \mathrm{ATP}$ (New England Nuclear, Boston, Massachusetts) using T4 polynucleotide kinase (Promega, Madison, Wisconsin) by standard methods. The oligonucleotide hybridization probes used in this study are shown in Table 2. Unincorporated nucleotides were removed by passage through Quick Spin Sephadex G-25 columns (Roche Diagnostics, Indianapolis, Indiana). Pre-hybridization and hybridization were at $32^{\circ} \mathrm{C}$ for 30 minutes and 24 hours, respectively, as described previously (Marconi et al., 1993). The temperature of $32^{\circ} \mathrm{C}$ was critical for the successful hybridization of oligonucleotide probes. Incubation of higher temperatures resulted in little or no hybridization (Marconi, personal communication). Following hybridization, the membranes were washed twice in $2 \mathrm{X}$ SSC (1X SSC is $0.15 \mathrm{M} \mathrm{NaCl}$ plus $0.015 \mathrm{M}$ sodium citrate) - $0.1 \%$ SDS at $32^{\circ} \mathrm{C}$ (10 minutes each) and once for 1 hour in $0.1 \mathrm{X}$ SSC- $0.1 \%$ SDS at $32^{\circ} \mathrm{C}$. The membranes were exposed to Kodak BioMax MS film with intensifying screens at $-70^{\circ} \mathrm{C}$. For removal of probe, the membrane was immersed in a boiling solution of $0.1 \mathrm{X}$ SSC- $1 \%$ SDS and was cooled for 30 minutes at room temperature. 
Table 2. Oligonucleotide hybridization probe or primer sequences

\begin{tabular}{|c|c|c|c|}
\hline Probe or Primer & Sequence ( $5^{\prime}$ to $\left.3^{\prime}\right)^{\mathrm{a}}$ & $\begin{array}{l}\text { Target } \\
\text { Gene }\end{array}$ & Region (bp) ${ }^{b}$ \\
\hline \multicolumn{4}{|l|}{ Southern analysis: } \\
\hline SOD3 & ATGTTTAAGCTGCCAGAACTTGG & sod & $1-23$ \\
\hline SOD4 & СТААТTAАТСАСТTСАТTGTAАAC & sod & $589-612$ \\
\hline \multicolumn{4}{|l|}{ Northern analysis: } \\
\hline 9R4 & CAGTATCTTTCAAATTGGCC ${ }^{a}$ & sod & $345-364$ \\
\hline OspA-5’ & GGCTGCTAACATTTTGCTTACATGCa & ospA & $46-70$ \\
\hline SecA & GTAAACCTCATCTGTAATCGCa & $\sec A$ & $1321-1341$ \\
\hline \multicolumn{4}{|l|}{ RT-PCR: } \\
\hline $\mathrm{P} 8 \mathrm{~F}$ & GGCAAAAGTTAAAACAGGAGG & sod & -26 to -6 \\
\hline P8R & GGCATGCTCCCAAACATC ${ }^{a}$ & sod & $490-507$ \\
\hline P11R & GGGCGGCTAATCATGTAGCa & $n a g B$ & $44-62$ \\
\hline P10F & GCATTATTGTTGGCAGATGAA & $\sec A$ & $101-121$ \\
\hline $\mathrm{P} 13 \mathrm{~F}$ & GGCTAAGCTAATGCAAATAGC & $\sec A$ & $2211-2231$ \\
\hline \multicolumn{4}{|l|}{ PE: } \\
\hline $\mathrm{P} 1$ & GTTCTTTATGAATTGGTTTAACATTCC & sod & -151 to -172 \\
\hline $\mathrm{P} 4$ & CATAACCAAGTTCTGGCAGC & sod & $9-28$ \\
\hline P5 & ACAGCATCATAATCATAACC & sod & $22-41$ \\
\hline $\mathrm{P} 14 \mathrm{R}$ & GAGCCAATAGTTGTCTCAAGTACTGa & $\sec A$ & $11-35$ \\
\hline P15R & CCAACGCTCAAGCTTATTAATA ${ }^{a}$ & $\sec A$ & $81-103$ \\
\hline \multicolumn{4}{|l|}{ DNA sequencing: } \\
\hline $\mathrm{P} 16 \mathrm{~F}$ & GTTGCCATTTTCATCAACATTAAC & $\sec A$ & -590 to -566 \\
\hline
\end{tabular}




\section{Reverse-transcriptase (RT) - polymerase chain reaction (PCR). Prior to reverse} transcriptase polymerase chains reaction (RT-PCR), the total RNA was further purified by treatment with DNase (Promega). RT-PCR was carried out with the Titan One Tube RTPCR System (Roche Diagnostics). Briefly, the reaction was carried out in a final volume of $50 \mu \mathrm{l}$ containing $0.2 \mathrm{mM}$ (each) dATP, dGTP, dCTP, and dTTP, $5 \mathrm{mM}$ dithiothreitol (DTT), $5 \mathrm{U}$ RNase inhibitor, $1.5 \mathrm{mM} \mathrm{MgCl} 2,0.3 \mathrm{mM}$ of each primer, $0.5 \mu \mathrm{g}$ RNA, and $5 \mathrm{U}$ Titan enzyme mix. The Titan enzyme mix contained avian myeloblastosis virus (AMV) - RT for first strand synthesis and an enzyme blend of Taq DNA polymerase and Pwo DNA polymerase for the PCR reaction. The reaction mixture was overlaid with $30 \mu \mathrm{l}$ of mineral oil. The RT step was carried out by programming the thermocycler (Perkin-Elmer, Boston, Massachusetts) as follows: 10 cycles of 30 seconds at $94^{\circ} \mathrm{C}$ (RNA denaturation), 30 seconds at $58^{\circ} \mathrm{C}$ (annealing), and 2 minutes at $68^{\circ} \mathrm{C}$ (elongation). Immediately following the $10 \mathrm{RT}$ cycles, the thermocycler was programmed for PCR with 25 additional cycles of 30 seconds at $94^{\circ} \mathrm{C}$ and 30 seconds at $58^{\circ} \mathrm{C}$. The elongation portion at $68^{\circ} \mathrm{C}$ of each PCR cycle added an additional 5 seconds; therefore, the elongation step began at 45 seconds and ended at 4 minutes. The amplified products were analyzed in $1.2 \%$ agarose gels and visualized by ethidium bromide staining.

Primer extension (PE) analysis. Transcriptional start sites for sod and $\sec A$ were analyzed by PE analysis as described previously (Marconi et al., 1993b). RNA extracted from exponential cultures of Sh-2-82 was hybridized with ${ }^{32} \mathrm{P}$-labeled oligonucleotide primers. The primer sequences used in this study are shown in Table 2. 
Extension reactions were performed with AMV-RT (Roche Diagnostics). The PE products, along with a sequencing ladder that contained the upstream region of the target gene, were loaded onto a 6\% acrylamide sequencing gel [7.5 M urea, $89 \mathrm{mM}$ Tris-borate, 0.025 M ethylenediaminetetraacetic acid (EDTA)]. The gel was electrophoresed for approximately four hours at a constant power of 55 watts. After electrophoresis, the gel was transferred to 3MM paper (Whatman, Hillsboro, Oregon) and dried for 1 hour at $75^{\circ} \mathrm{C}$ using a BioRad model 583 gel dryer (BioRad). Dried gels were exposed overnight to Kodak BioMax MR film at $-70^{\circ} \mathrm{C}$. The transcriptional start sites were identified by the approximate size of the PE product as compared with the sequencing ladder.

Polymerase chain reaction (PCR). The DNA template for secA sequencing ladder was generated using Titan PCR System (Roche Diagnostics). The conditions for the reaction were the same as RT-PCR described above except the RT step was omitted. The PCR product was extracted from the agarose gel and purified using the QiaQuick kit (Qiagen, Valencia, California).

DNA sequencing. Manual DNA sequencing by the dideoxy chain-termination method (Sanger et al., 1977) was performed using the T7 Sequenase Version 2.0 DNA sequencing kit (United States Biochemical, Cleveland, Ohio) or the Fidelity DNA sequencing system (Oncor, Purchase, New York) according to manufacturers directions. The DNA template used for the sequencing ladder in the secA PE analysis was generated by PCR using primers P16F and SecA (Table 2). The pCW100 recombinant plasmid was used in sod PE analysis. This plasmid contained a 609-bp ORF encoding sod, as well as portions of secA 
upstream and $o s p B$ downstream (Whitehouse et al., 1997). The DNA sequencing products were labeled with $\left[\alpha-^{35}\right.$ S ATP and heated at $75^{\circ}$ for 5 minutes before loading onto a $6 \%$ acrylamide sequencing gel as described above.

Ribonuclease protection assay (RPA). The simultaneous detection of borrelial secA and sod transcripts was accomplished by RPA using the Multi-NPA kit (Ambion, Austin, Texas). Biotinylated probes (Genosys, Woodlands, Texas) and were hybridized for four hours at $37^{\circ} \mathrm{C}$. The sequences and locations of each probe are shown in Table 3 (Fraser et al., 1997). Nonhybridized probe and RNA were digested with nuclease mixture containing S1 nuclease, RNase A, and RNase T1. The resulting protected fragments were precipitated with ethanol and dissolved in gel loading buffer (95\% formamide, 0.025\% xylene cyanol, 0.025\% bromophenol blue, $18 \mathrm{mM}$ EDTA, and 0.025\% SDS). The fragments were resolved in 15\% PAGE containing $8 \mathrm{M}$ urea in TBE running buffer (0.9 M Tris, $0.9 \mathrm{M}$ boric acid, and $20 \mathrm{mM}$ EDTA) at 10 volts per centimeter between electrodes. The gel was transferred to a positively-charged nylon membrane by electroblotting using the BioRad Protean II electroblotting apparatus (BioRad) or the BioRad Semi-Dry Transfer Apparatus (BioRad). The nucleic acid fragments were crosslinked to the membrane by baking at $80^{\circ} \mathrm{C}$ for 15 minutes. The bands of full-length probe and protected fragments were visualized by development with the BrightStar BioDetect Kit (Ambion) and exposure to Kodak BioMax CL film. The detection was dependent upon the degree of chemiluminescence. The intensity of bands was quantified using Quantity One software program (BioRad). 
Table 3. Biotinylated oligonucleotide probes used in ribonuclease protection assays

\begin{tabular}{llll}
\hline Gene & Nucleotide sequence $^{\mathrm{a}}$ & Size & Location $^{\mathrm{b}}$ \\
\hline secA & AAAAAAAAACCCACTTCCACAATAACAA & 84 bases & $2578-2662$ \\
& GGCTCATTTCTGCCTATTTTAGGAGAACT & & \\
& TCTAACCACTTGAACATTAGAAGCGC & & \\
sod & AAAAAAAAAAGCATTGAAATCACTTTAA & 66 bases & $384-450$ \\
& GGCCACTCTCAGGACACAATACTAACCAT & & \\
& GCCCAACC & & \\
& AAAAAAAAAACTCCGCCTTGAGAAGGTGC & 53 bases & $421-474$ \\
& TGTAGCAGGTGCTGGCTGTT & & \\
\hline
\end{tabular}

${ }^{a}$ The antisense probes contain an additional 10 adenines (A). These nucleotides have been added to the sequence to distinguish the protected size fragment from the full-length probe.

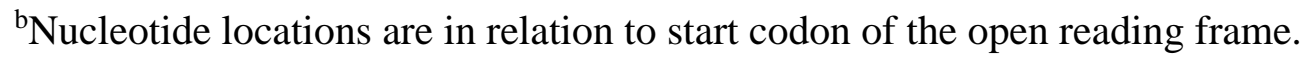


Secondary structure analysis. Potential secondary structures of the $\sec A$ and sod promoter regions were assessed using Mfold software, version 2.0 (Jaeger et al., 1990). The RNA sequence entered into the program arbitrarily included 50 nucleotides upstream from each transcriptional start site identified by $\mathrm{PE}$ analysis. In this way, the total number of nucleotides to be folded was similar for each promoter. The secA and sod promoter upstream regions were compared to the well-characterized promoter of ospA, which is transcribed from a $\sigma^{70}$-promoter sequence (Howe et. al., 1986).

Preparation of cell free extracts (CFE). B. burgdorferi was cultured in BSK-II or BSK-E media to stationary phase and harvested by centrifugation at 20,250 x $g$ for 20 minutes at $4^{\circ} \mathrm{C}$. Cell pellets were washed twice in $25 \mathrm{mM}$ sodium chloride- $20 \mathrm{mM} \mathrm{N}$-2hydroxyethylpiperazine-N-2-ethanesulfonic acid (HEPES), pH 7.6 (Carroll and Gherardini, 1996). Cells were resuspended in a minimal volume of HEPES buffer for sonication using a Branson model 250 sonifier (Branson Ultrasonics Corporation, Danbury, Connecticut) equipped with a high-intensity cup-horn. Sonic settings were adjusted to 20-second bursts with an output of 70 watts. Sonication was carried out until no spirochetes were observed by dark-field microscopy. A constant temperature of $4^{\circ} \mathrm{C}$ was maintained by continuous circulation of cold water through the cup horn. Cellular debris were removed by centrifugation at $15,500 \times \mathrm{g}$ for 20 minutes at $4^{\circ} \mathrm{C}$. Likewise, E. coli EMG-2 cells were harvested at stationary phase and washed twice in $25 \mathrm{mM}$ sodium chloride and $20 \mathrm{mM}$ HEPES before sonication. Cells were disrupted by six 20-second cycles at 70 watts and cellular debris were removed by centrifugation as described above. Protein concentrations of 
CFE were determined using BCA Protein Kit (Pierce, Rockford, Illinois) with bovine serum albumin as a standard.

SDS-polyacrylamide gel electrophoresis (PAGE). B. burgdorferi was cultured in BSK-II or BSK-E media to stationary phase and harvested by centrifugation $(27,500 \mathrm{x}$ g, 20 minutes, $4^{\circ} \mathrm{C}$ ). Cells were washed twice in $25 \mathrm{mM} \mathrm{NaCl-20mM} \mathrm{HEPES,} \mathrm{pH} \mathrm{7.6.} \mathrm{For}$ SDS-PAGE, samples were mixed with an equal volume of loading buffer (100 mM Tris-HCl [pH 6.8], 200 mM DTT, 4\% SDS, 0.2\% bromophenol blue, 20\% glycerol) and heated at $100^{\circ} \mathrm{C}$ for 5 minutes (Laemmli, 1970). Twenty micrograms of protein from the CFE were added per lane and resolved with 10\% PAGE in Tris-Glycine running buffer at 10 volts per centimeter between electrodes. After electrophoresis, protein bands were first stained with Coomassie blue, followed by silver staining using the SilverXpress stain kit (BioRad).

\section{Non-denaturing polyacrylamide gel electrophoresis (PAGE) and staining for}

SOD activity. Proteins in the CFE were separated by PAGE in 10\% acrylamide gels under nondenaturing conditions (Davis, 1964). Approximately $400 \mu \mathrm{g}$ of borrelial CFE protein was added per lane and electrophoresed at 10 volts per centimeter between electrodes. As a positive control, $50 \mu$ g of E. coli CFE protein was added per lane. Staining for SOD activity in gels was done as described by Beauchamp and Fridovich (1971). This method was based on the enzymatic inhibition of the reduction of nitroblue tetrazolium (NBT) dye by $\mathrm{O}_{2}^{-}$to formazan. The gel was immersed in 2.45 mM NBT for 20 minutes, and then placed in a solution containing $28 \mu \mathrm{M}$ riboflavin, $28 \mathrm{mM} N, N, N^{\prime}, N^{\prime}$-tetramethylethylenediamine 
(TEMED), and $36 \mathrm{mM}$ potassium phosphate (pH 7.8) for 15 minutes in the dark. The gel was then exposed to fluorescent white light until there was full development of the deep blue monoformazan color. Areas containing SOD block the $\mathrm{O}_{2}{ }^{-}$dependent reaction and appear as achromatic bands in gels. Fe-, Mn-, and CuZn-containing SODs were distinguished by treating duplicate gels with $0.1 \mathrm{mM}$ EDTA plus either $5.0 \mathrm{mM} \mathrm{H}_{2} \mathrm{O}_{2}$ (Kirby et al., 1980) or $1.0 \mathrm{mM}$ NaCN (Rotilio et al., 1972).

Spectrophotometric analysis of SOD activity. Superoxide dismutase activity was assayed by the xanthine oxidase-cytochrome $c$ method of McCord and Fridovich (1969). Briefly, cytochrome $c$ (Sigma) was reduced by the $\mathrm{O}_{2}{ }^{-}$generated by xanthine oxidase (Sigma). A Beckman DU-650 spectrophotometer (Beckman-Coulter Instruments) equipped with enzyme kinetics software was used for these measurements. One unit of SOD activity was defined as the amount of enzyme required to inhibit the rate of reduction of cytochrome c by $50 \%$. Specific activity was determined as units per mg of CFE protein.

Two-dimensional (2D) gel electrophoresis. For 2D nonequilibrium $\mathrm{pH}$ gradient electrophoresis (NEPHGE), stationary phase cells were washed in HEPES buffer as described above and centrifuged at 15,500 x $g$ for 10 minutes. Pellets were solubilized directly in NEPHGE sample buffer (7M urea, 2\% CHAPS, 10 mM DTT, 2\% ampholytes 310 in distilled $\mathrm{H}_{2} \mathrm{O}$ ) for two hours at room temperature, followed by centrifugation at 15,500 $\mathrm{x} g$ for five minutes to remove cell debris and precipitated DNA. Protein concentrations were determined using the RC-DC protein assay (BioRad), which is a colorimetric assay for protein determination in the presence of reducing agents and detergents. A total of $100 \mu \mathrm{g}$ 
protein was loaded onto 18-cm pre-cast pH 6-8 tube gels (Genomic Solutions, Ann Arbor, Michigan). The electrophoretic conditions in the 2D-NEPHGE procedure were a modification of the technique described by O'Farrell et al. (1977). Briefly, the gels were electrophoresed for six hours at $400 \mathrm{~V}$ at $20^{\circ} \mathrm{C}$ using $20 \mathrm{mM} \mathrm{NaOH}$ cathode buffer and 10 $\mathrm{mM} \mathrm{H}_{3} \mathrm{PO}_{4}$ anode buffer. The tube gels were placed in equilibration buffer [0.06 M Tris$\mathrm{HCl}$ (pH 6.8), 2\% SDS, $100 \mathrm{mM}$ DTT and 10\% glycerol] for 15 minutes at room temperature. The gels were stored at $-20^{\circ} \mathrm{C}$ until used.

High-resolution 2D-PAGE with immobilized pH gradients (IPGs) in the first dimension was performed as described by Westermeier (1997). Stationary phase cells were harvested as described above. Pellets were solubilized in 2D-lysis buffer I (50 mM Tris, $0.3 \%$ SDS, and $65 \mathrm{mM}$ DTT). After a 30 -minute incubation at $37^{\circ} \mathrm{C}$, protein concentrations were determined using RC-DC protein assay (BioRad). One hundred micrograms of protein were placed in 2D sample buffer II (9M urea, 2\% CHAPS, 65 mM DTT, 1\% pH 3-10 ampholytes, $0.01 \%$ bromophenol blue) and incubated for 30 minutes at room temperature. The protein samples were centrifuged at $15,500 \mathrm{x} g$ for one minute to remove any precipitating cell debris. The solubilized protein sample was transferred to an IPG strip pH 5-9 (Amersham-Pharmacia Biotech, Piscataway, New Jersey) in an IPG rehydration tray (Amersham-Pharmacia Biotech). The loaded IPG strip was overlaid with mineral oil and incubated overnight. Isoelectric focusing (IEF) was carried out for $48 \mathrm{kVh}$ (18-24 hours, $20^{\circ} \mathrm{C}, 10 \mathrm{~mA}$ ) using a Multiphor II electrophoresis unit (Amersham-Pharmacia Biotech) following manufacturer’s instructions. The IPG strip was equilibrated with $15 \mathrm{ml}$ equilibration buffer [50 mM Tris-HCl (pH 8.8), $6 \mathrm{M}$ urea, 30\% glycerol, 1\% w/v DTT and $1 \% \mathrm{w} / \mathrm{v}$ SDS] for 30 minutes prior to separation by SDS-PAGE. 
For the second dimension, NEPHGE gels or IEF gels were loaded and run on a 10\% acrylamide Laemmli SDS-PAGE gel (Genomic Solutions) at $15 \mathrm{~W}$ per gel until the bromophenol blue dye front was within one centimeter of the base of the gel. Gels were stained using the Investigator silver stain kit (Genomic Solutions), which is a modification of the Rabilloud (1992) procedure. Glutaraldehyde was omitted from the procedure to allow for mass spectrophotometer profiling (Morrisey, 1981). Images of silver-stained gels were scanned using a Personal Densitometer SI (Molecular Dynamics, Sunnyvale, California).

In-gel tryptic digestion of proteins. Excision and preparation of protein spots from silver-stained gels were performed as previously described (Hellman et al., 1995; Rosenfeld et al., 1992). Excised gel pieces from silver-stained gels were washed and sonicated twice in $50 \mu \mathrm{M}$ ammonium bicarbonate in 50\% acetonitrile (ABC buffer) for five minutes. Three microliters of $45 \mathrm{mM}$ DTT and $100 \mu \mathrm{l} 50 \mu \mathrm{M}$ ABC buffer were added to the gel pieces and incubated at $37^{\circ} \mathrm{C}$ for twenty minutes to reduce the proteins sequestered in the gel. The proteins were alkylated by incubating the gel pieces in four $\mu \mathrm{L}$ of $100 \mathrm{mM}$ iodoacetamide for twenty minutes in the dark. Gel pieces were washed in ABC buffer; the supernatant was removed, and the gel pieces were dried by vacuum using a Speed-Vac (Savant, Saint Paul, Minnesota). Fifteen microliters of porcine trypsin (0.02 $\mu \mathrm{g} / \mu \mathrm{L}$; Promega) were added and incubated overnight at $30^{\circ} \mathrm{C}$ for in-gel digestion of proteins. Sufficient ABC buffer was added as necessary to completely immerse gel pieces. Following digestion, the gel pieces were sonicated in $0.1 \%$ trifluoroacetic acid in $60 \%$ ammonium bicarbonate twice and the supernatant fluids containing the released peptide fragments were collected. The combined supernatant fluids were dried and were submitted for mass spectrophotometry analysis. The 
tryptic peptides were resuspended in $\alpha$--cyano-4-hydrooxycinnamic acid matrix (HewlettPackard, Palo Alto, California) and analyzed on a ToFSpec SE matrix-assisted laser desorption ionization time-of-flight (MALDI-TOF) mass spectrometer equipped with a nitrogen laser. The resulting spectra were searched against the B. burgdorferi genome sequence in the NCBInr.10.17.98 database (www.ncbi.nlm.nih.gov) using MS-Tag software available via the internet (http://prospector.ucsf.edu).

Computer imaging. Figures of gels and blots were assembled and labeled using Adobe Photoshop version 4.0 or Corel Draw version 8.0. Growth curve data were analyzed using Sigma Plot version 5.0 (Jandel, Chicago, Illinois). Two-dimensional gel images were generated using ImageQuant software (Molecular Dynamics) and analyzed using Z3 software (Compugen, Tel Aviv, Israel).

Statistical analysis. Growth curve data were recorded in Excel 5.0 software (Microsoft, Redmond, Washington). Comparisons between two populations, the test condition and the standard condition, were analyzed by the Student $t$ test using the statistical package in Excel 5.0. 


\section{RESULTS}

\section{Chapter 1 - Transcriptional analysis of $\operatorname{sod}$ of $B$. burgdorferi}

Detection of secA-sod transcript. The sod gene in B. burgdorferi Sh-2-82 consists of a 609-bp ORF, which encodes a $0.6 \mathrm{~kb}$ mRNA transcript, if the gene is monocistronic. The size of the sod transcript was determined by Northern blot analysis of total RNA. Borrelial RNA was extracted from early stationary phase cultures of Sh-2-82 as previously described in the Materials and Methods. Figure 1A shows the separation of borrelial RNA and staining with ethidium bromide. Distinct bands of 23S and 16S ribosomal RNA (rRNA) were observed, indicating that the RNA had not been degraded during isolation. The oligonucleotide probe 9R4, specific for sod, was derived from the DNA sequence of the recombinant plasmid pCW100 and is shown in Table 2. The 9R4 probe hybridized to a major band of approximately 3.7 kb (Figure 1B). Similarly, a 3.7 kb transcript was observed with a secA-specific probe. This mRNA transcript is of sufficient size to encode both proteins. An additional 2.7-kb band was observed with the secA probe, which corresponds to the 2,697 bp ORF of $\sec A$. As a control, the blot was also probed for ospA, one of the dominant surface antigens of $B$. burgdorferi. Cotranscription of ospA (820 bp) and ospB (888 bp) from a common promoter has been reported (Howe et al., 1986). As expected, a single mRNA transcript of approximately $1.8 \mathrm{~kb}$ was observed.

The possibility of cotranscription was further examined by RT-PCR. Pairs of primers specific to each individual gene (P8F/P8R) for sod-sod; P10F/secA for secA- 
Figure 1. Northern blot analysis of Sh-2-82 mRNA. Total RNA (10 $\mu \mathrm{g})$ isolated from $B$. burgdorferi Sh-2-82 was electrophoresed in a glyoxal agarose gel and transferred onto a nylon membrane. The blot was probed sequentially with the ${ }^{32} \mathrm{P}$-labeled oligonucleotides indicated in lanes 1-3. Lane M, RNA molecular size standards (Gibco BRL). The blot is representative of results of three independent experiments. 


\section{A. Agarose gel B. Northern blot}

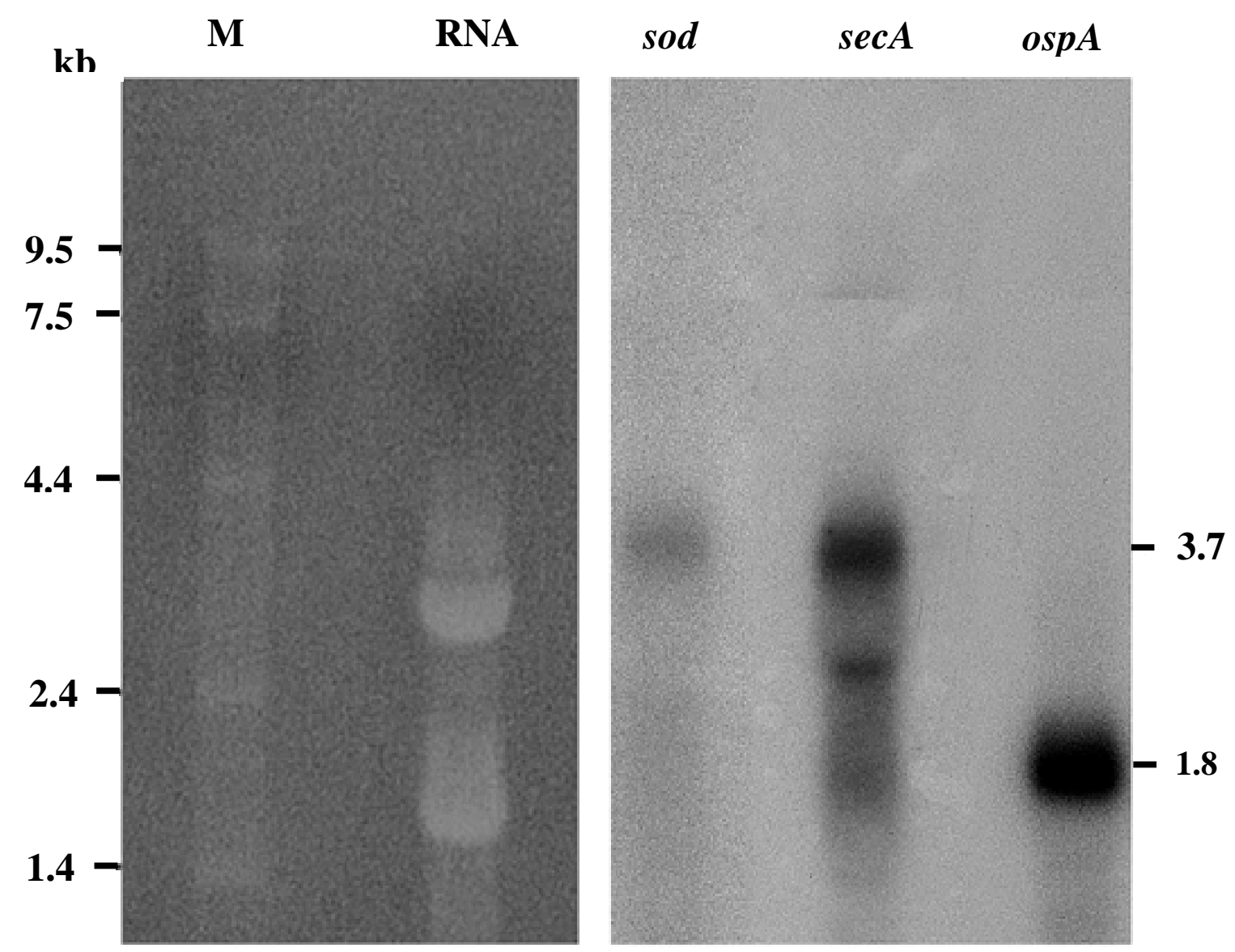


secA) or to the two adjacent genes (P13F/P8R for secA-sod) were chosen (Figure 2A).

Products from each individual gene confirmed that the message could be detected in the RTPCR assay (Figure 2B, lanes 2 and 3). In addition, primers P8F/P11R for sod-nagB, which are transcribed in opposite directions, were used to detect any DNA contamination in the RNA samples. This control did not yield products (Figure 2B, lane 5). The secA-sod region was positive for amplification from RNA, yielding a product of the predicted size of $1011 \mathrm{bp}$ (Figure 2B, lane 4). These results indicated that these genes are transcribed as a single polycistronic mRNA.

Determination of $\sec A$ and $\operatorname{sod}$ promoter. To locate the promoter of $\operatorname{sod}$ and $\sec A$, the transcriptional start site was analyzed by PE. In preliminary experiments prior to the RTPCR analysis, the upstream sequence of sod derived from pCW100 was analyzed for -10 and -35 promoter elements. The promoters recognized by both $\sigma^{70}$ and rpoS in E. coli have a Pribnow box with a consensus of 5'-TATAAT and a -35 promoter sequence with a consensus of 5'-TTGACA. Several sequences similar to these $\sigma^{70}$-like elements were identified in the borrelial DNA on the basis of homology. Three primers, P1, P4, and P5, were used in PE analyses (Table 2 and Figure 3). Of the three primers tested, only the P4R primer generated a PE product. Manual dideoxy sequencing was performed using pCW100 as a sequencing template. A prominent band migrated with an A residue 265 nucleotides upstream from the ATG translational start codon (Figure 4A), indicating that the first base of the transcript was the corresponding $\mathrm{T}$ residue. A minor band was also observed at the $\mathrm{G}$ residue 274 nucleotides from the translational start codon. These locations suggested that if a sod transcript started from this site, it would be approximately 270 nucleotides. 
Figure 2. RT-PCR analysis of the sod transcript. A. Location and direction of primers used in RT-PCR. B. RT-PCR analysis. The amplified products were obtained from pairs of primers, which span intragenic regions of sod (533 bp; lane 2) and secA (1240 bp; lane 3) and the intergenic region of secA-sod (1011 bp; lane 4). Negative controls included: primers derived from sod and nagB, which are transcribed in opposite directions (lane 5); no RNA template (lane 6); and no enzymes (lane 7). The positive control used specific primers for $\beta$ actin that were provided in the RT-PCR system (Roche Diagnostics) (587 bp; lane 8). Lane M, AmpliSize DNA size standards (BioRad). 
A.

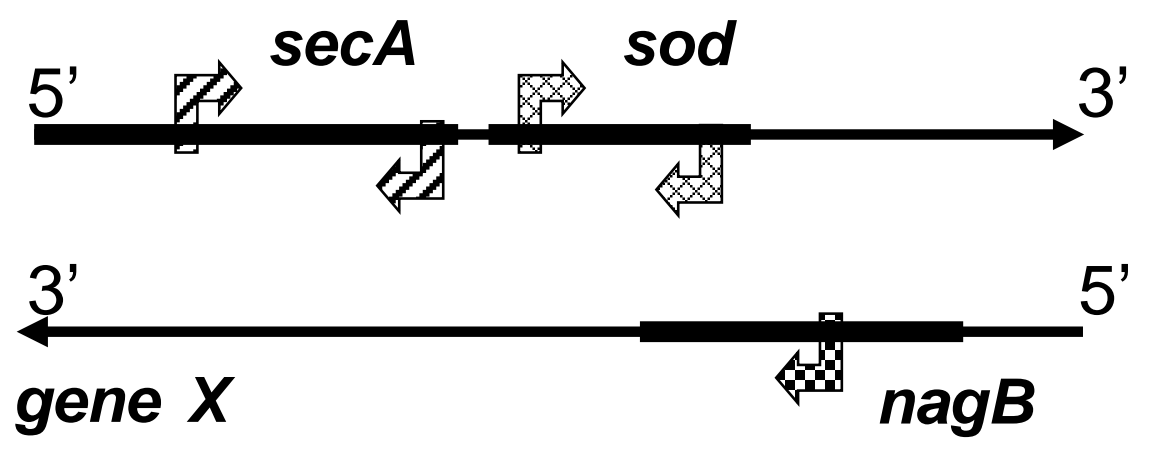

B.

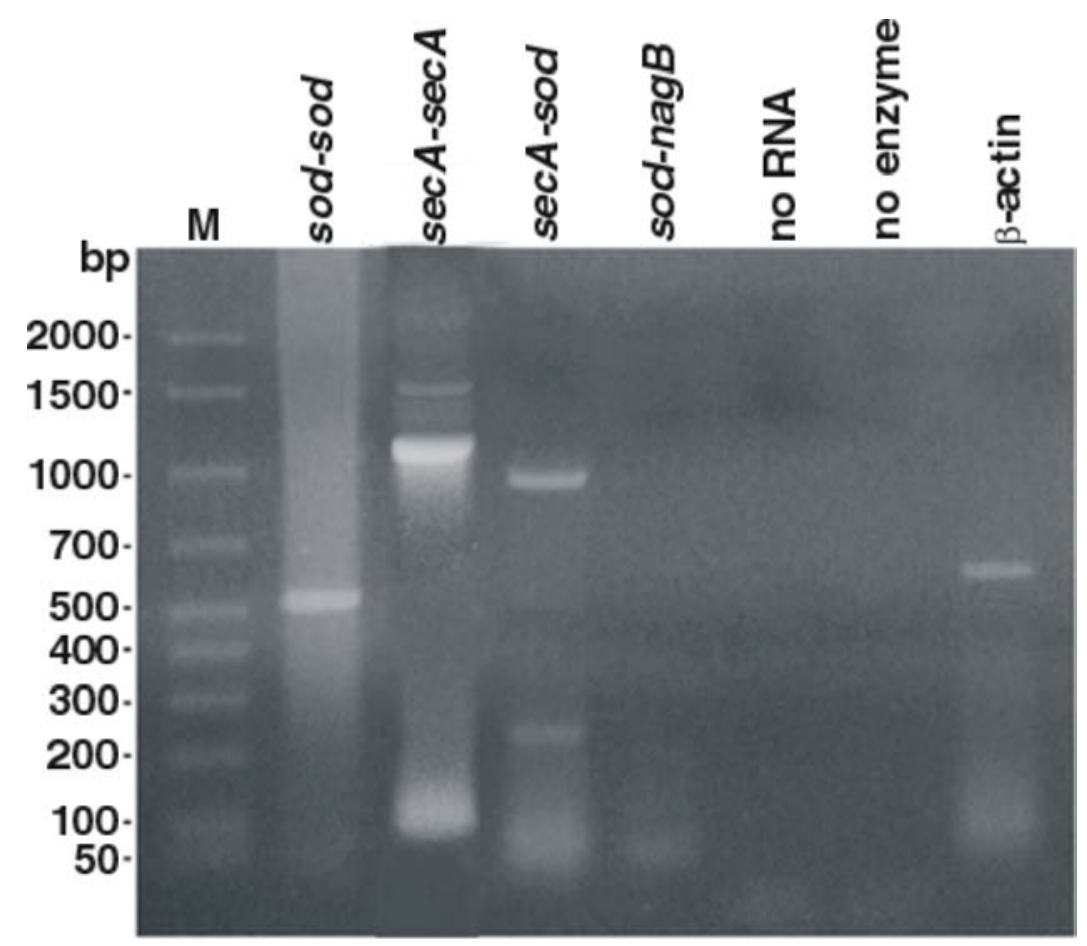


Figure 3. Approximate locations of primers used in primer extension analyses. The DNA segment represents the coding (plus) strand for the secA and sod genes. All primers were the reverse complement of the coding strand. 


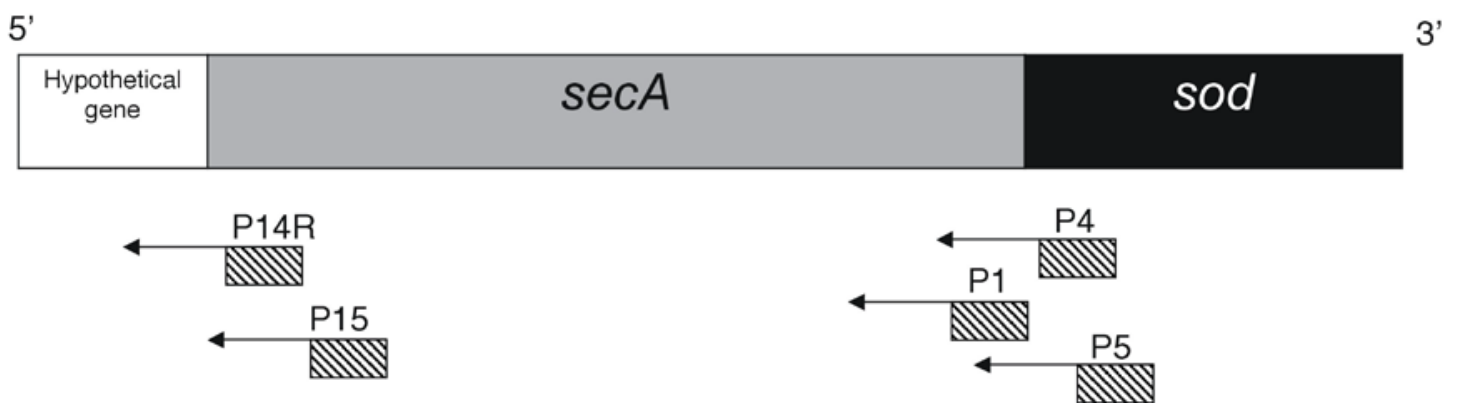


Figure 4. Lack of $\sigma^{70}$-like promoter region upstream of $B$. burgdorferi sod. A. Primer extension analysis. The $\mathrm{P}_{\mathrm{ex}}$ lane contains the extension products obtained when total RNA from Sh-2-82 was primer extended with reverse transcriptase and 5'-end labeled P4R primer. The same primer was used to generate the sequencing ladder with pCW100 DNA as a template. Transcriptional start site is labeled +1 . B. Nucleotide sequence of region upstream of sod. Shine-Dalgarno sequence, lower case; sod transcriptional start site, +1 ; putative -10 promoter sequence, underlined; secA stop codon, ***; putative Fur-binding box, boxed. The deduced amino acid sequence for the 5'-coding region of sod is shown below the nucleotide sequence. These sequence data appear in GenBank under the accession number AF011226. C. Comparison of the sequence of the putative sod promoter with those promoter sequences of the following: osp $A B$, ospC, flaA, flaB, flgK of $B$. burgdorferi $(B b)$; and sodA of Haemophilus influenzae $(\mathrm{Hi})$. 


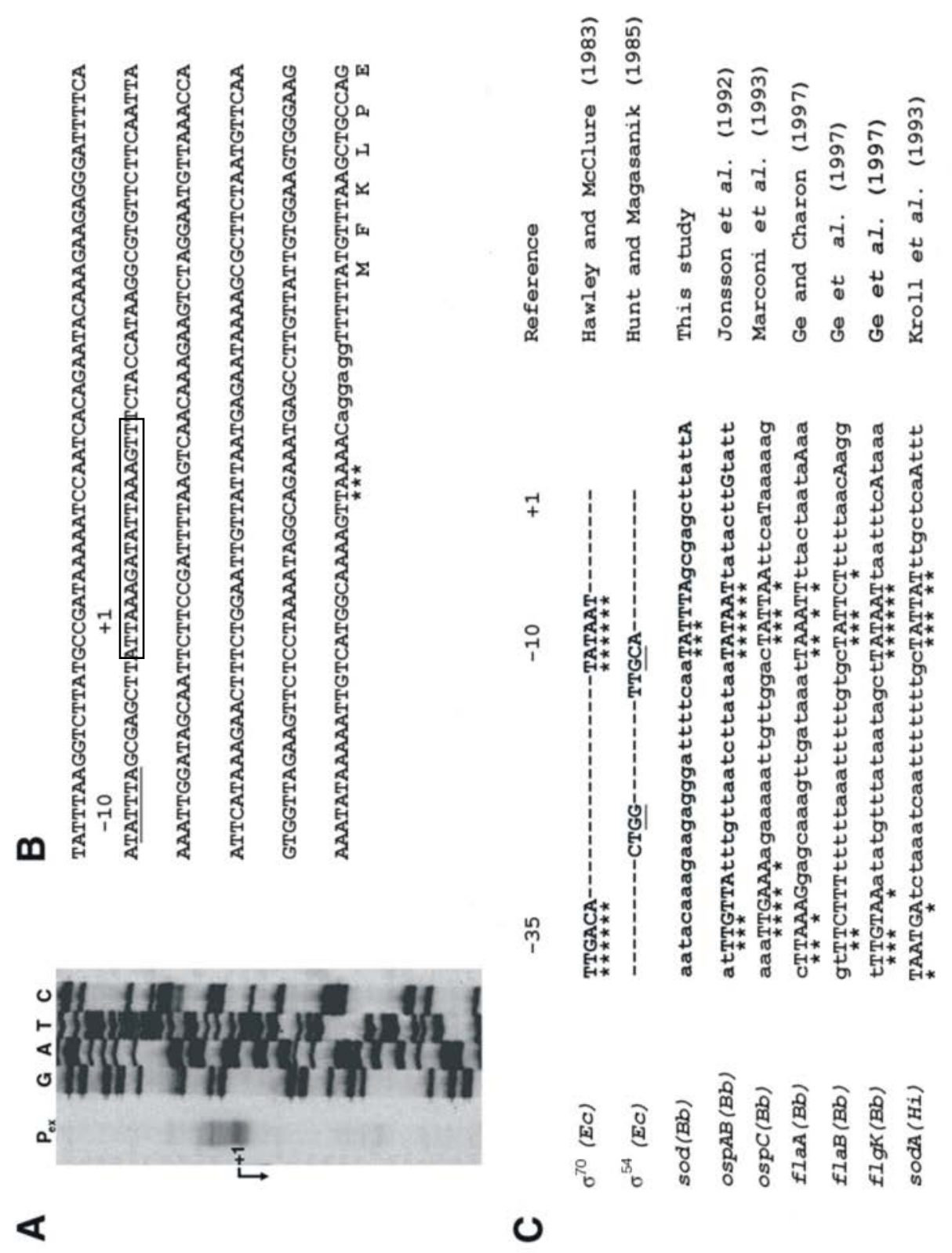


The sequence around the deduced -10 region of sod revealed a putative $\sigma^{70}$-like Pribnow box (TATTTA) 11 nucleotides upstream of the major start site (Figure 4B). This -10 hexamer had five out of six nucleotides or four out of six nucleotides identical to the -10 sequence for ospC of B. burgdorferi or sodA of Haemophilus influenzae, respectively (Figure 4C). However, this -10 hexamer had only three out of six nucleotides identical to the $\sigma^{70}$ Pribnow box. In addition, a sequence resembling the -35 region of the $\sigma^{70}$ recognition sequence was not evident. Doublets of GG and GC in the highly conserved -23/-11 region of the $\sigma^{54}$ recognition site were not identified. This lack of consensus for $\sigma^{54}$ is consistent with the genomic analysis described by Studholme and Buck (2000). These results, along with the lack of PE products with the primers P1 and P5, suggested the absence of either a $\sigma^{70}$-like promoter or a $\sigma^{54}$-like promoter immediately upstream of sod.

With the results of the RT-PCR, the presence of a promoter upstream of $\sec A$ was investigated by PE using primers P14 and P15 (Table 2 and Figure 3). In initial experiments, the sequencing template for secA was derived from the borrelial clone, pGMMGB26, described in the TIGR database (www.tigr.org). After several unsuccessful attempts resulting in a PE product without a DNA sequence, it was decided that the clone did not contain sufficient secA sequence to which the primer could bind. Using PCR, a 1031-bp fragment (Figure 5) was generated from Sh-2-82 genomic DNA with primers P16F and SecA (Table 2 and Figure 3)), which encompassed a 590-bp region upstream of the start codon of $\sec A$. The secA amplified fragment was used directly as a template for sequencing without additional cloning. The sequence generated and location of the PE product using the primer 
Figure 5. PCR using SecA reverse primer and P16F forward primer. A. Location of primers used to generate 1031- bp fragment including 441 bases upstream of secA start codon and 590 bases of the 5'end of the 2697 bases of the secA gene. B. PCR reaction using the reverse primer, SecA, and forward primer, P16F. Lane 1, Molecular weight markers. Lane 2, PCR product of SecA and P16F. 
A.

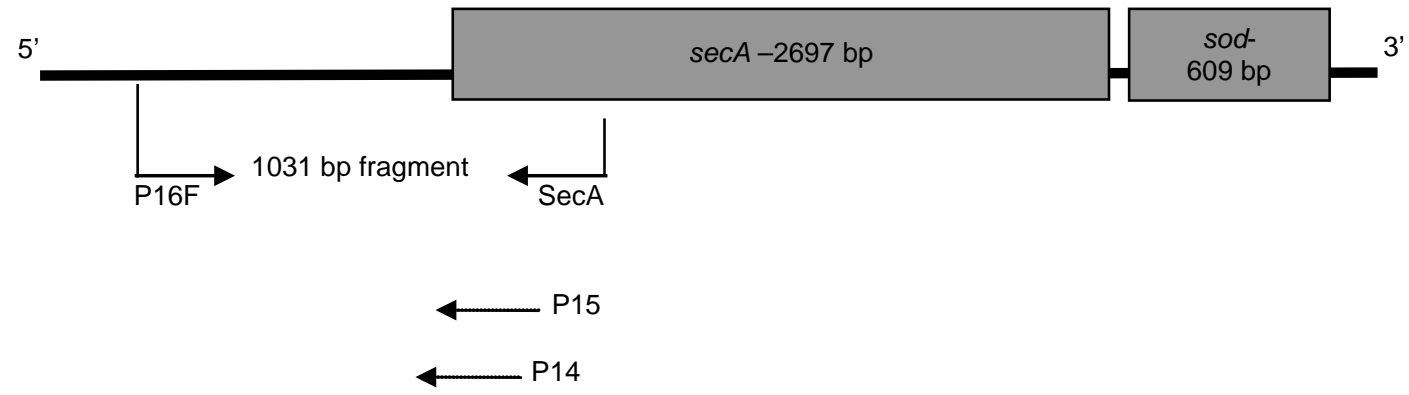

B.

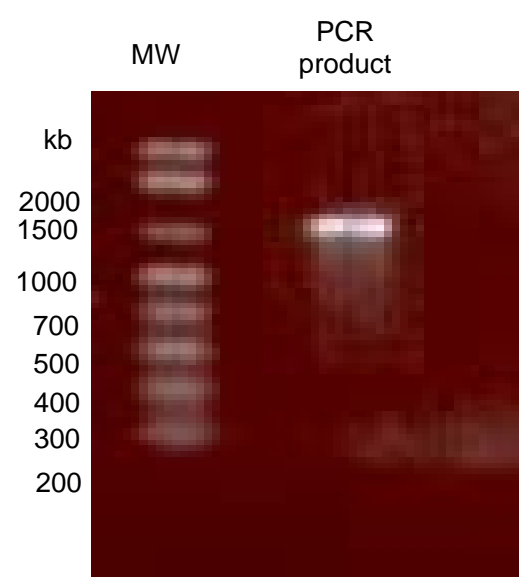


P15 are shown in Figure 6A. A prominent band migrated with an A residue 100 nucleotides upstream from the ATG translational start codon, indicating that the first base of the transcript was the corresponding T residue. The same PE product was observed with an independent primer, P14 (data not shown). The sequence of the deduced -10 hexamer is $100 \%$ identical to the $E$. coli consensus Pribnow box and the -10 sequences for osp $A B$ and flgK of B. burgdorferi (Figure 6B). Scanning for an optimal spacing of 17 nucleotides upstream identified a -35 region (TTTAAA) with four out of six nucleotides identical to promoters recognized by either $\sigma^{70}$ or $r p o S$. A similar degree of variation in the -35 region has been reported for other borrelial promoters (Figure 5C). These results suggested that a typical $\sigma^{70}$-type promoter is involved in the transcription of the secA-sod operon.

The above results have been published recently. See: Nichols, T. L., C. A. Whitehouse, and F. E. Austin. 2000. Transcriptional analysis of a superoxide dismutase gene of Borrelia burgdorferi. FEMS Microbiol. Lett. 183:37-42. 
Figure 6. Putative promoter region of $B$. burgdorferi secA-sod operon. A. PE analysis. The $\mathrm{P}_{\mathrm{ex}}$ lane contains the extension products obtained when total RNA from Sh-2-82 was primer-extended with reverse transcriptase and 5'-end labeled P15R primer. The same primer was used to generate the sequencing ladder with a 1931-bp PCR product

encompassing the region upstream of $\sec A$ as a template. Transcriptional start site is labeled +1 . B. Nucleotide sequence of region upstream of secA. Shine-Dalgarno sequence, lower case; sec $A$ transcriptional start site, +1 ; putative -10 promoter sequence, underlined; putative -35 promoter sequence, double underlined; and putative Fur-binding box, boxed. The deduced amino acid sequence for the 5'-coding region of secA is shown below the nucleotide sequence. These sequence data appear in GenBank under the accession number AF011226. C. Comparison of the sequence of the putative $\sec A$ promoter with those promoter sequences of the following $B$. burgdorferi genes: ospAB, ospC, flaA, flaB and $f l g K$. 

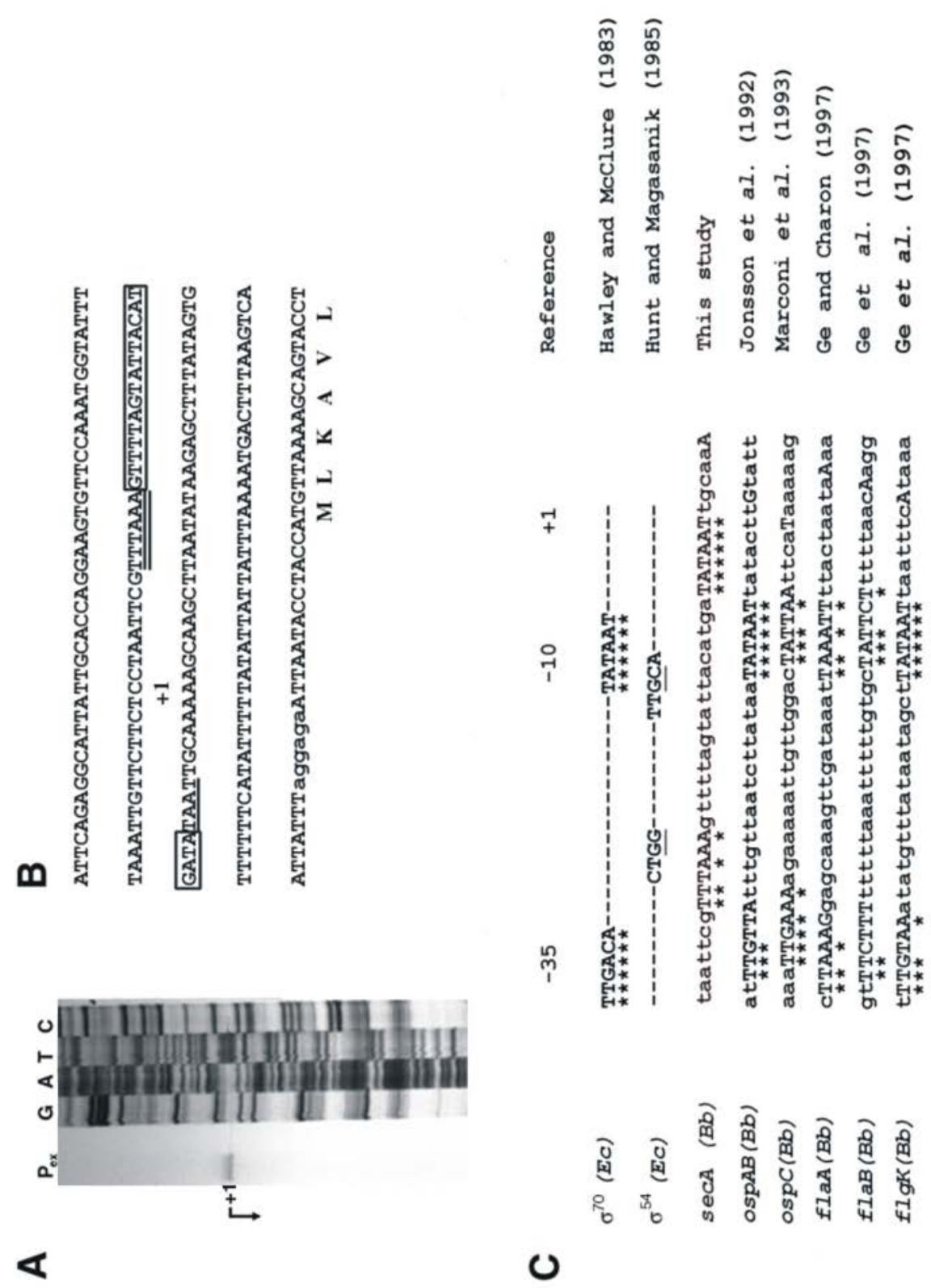
Predicted secondary structure of $\sec A$ and sod mRNA transcripts. To complete the characterization of the secA-sod promoter, the mRNA transcripts were examined for the involvement of secondary structure in the initiation of transcription. Because no promoter consensus could be discerned in the vicinity of position -270 of the sod transcript (Figure 4), the possibility existed that the PE product detected was due to a site of a structural impediment to PE by RT. Figure 7 shows the predicted secondary structure of $\sec A$ and $\operatorname{sod}$ putative promoter regions as determined by M-fold software. Sequence from ospA was used as a control (Fraser et al., 1997). The program analyzed approximately 119 bases of RNA sequence that encompassed the putative transcriptional start sites of $\sec A$ and sod for optimal folding. The folding energies used to calculate the secondary structure for the RNA molecule were similar: osp $A, \Delta \mathrm{G}=-15.3 \mathrm{kcal} \mathrm{mol}^{-1}$; $\sec A, \Delta \mathrm{G}=-14.0 \mathrm{kcal} \mathrm{mol}^{-1} ; \operatorname{sod}, \Delta \mathrm{G}=$ $-15.2 \mathrm{kcal} \mathrm{mol}^{-1}$. The sequences of ospA and $\sec A$ appeared to have little secondary structure, with long linear stretches in the -10 and -35 regions (Figure $7 \mathrm{~A}$ and $7 \mathrm{~B}$ ). In contrast, sod appeared to have more secondary structure with a stem-loop beginning at position -21 (Figure 7C). In relation to the transcriptional start site identified by PE analysis (Figure 4), the -35 region would be located in this stem-loop. Therefore, the PE product identified in sod may be artifactual due to the inability of RT to traverse this apparent secondary structure. These findings supported the conclusion that sod is transcribed from the $\sec A$ promoter as a single transcriptional unit, secA-sod. 
Figure 7. Predicted secondary structure using Mfold analysis. A. ospA, B. $\sec A$, C. $\operatorname{sod}$. The -10 regions are underlined; the -35 regions are double-underlined; and the transcriptional start site is denoted by +1 ; $n=$ number of nucleotides; $\Delta \mathrm{G}=$ free energy calculation based on the predicted folding pattern. 


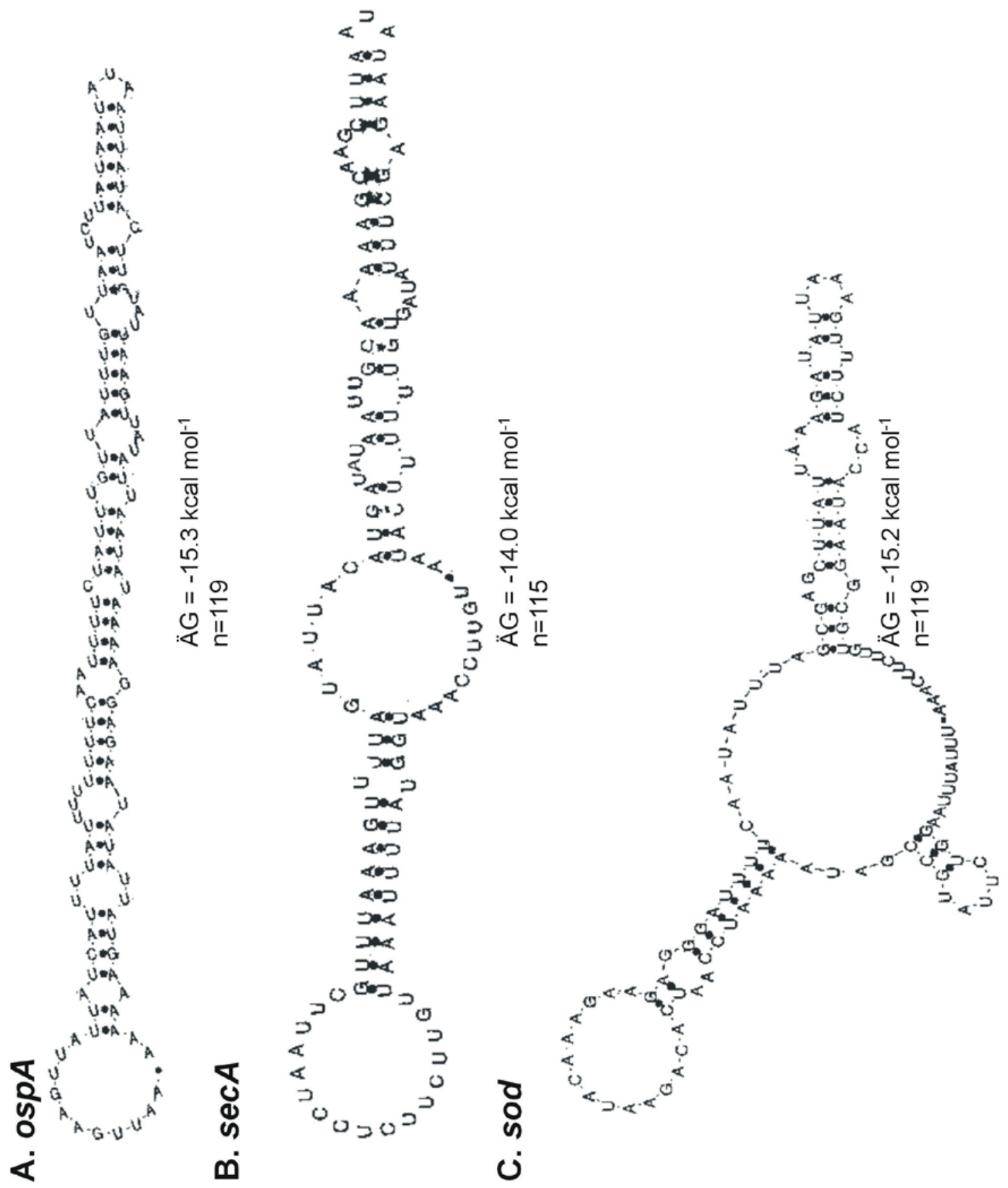




\section{Chapter 2- Identifying a possible role for iron in B. burgdorferi}

Identification of Fur-binding boxes. Fur, in the presence of divalent metals such as

$\mathrm{Fe}^{2+}, \mathrm{Mg}^{2+}, \mathrm{Cu}^{2+}$, or $\mathrm{Zn}^{2+}$, represses the transcription of genes involved in Fe assimilation and negatively regulates sodA expression. Metal-bound Fur binds a consensus sequence called a Fur box located within the promoter region of the gene. There are two methods for identification of Fur boxes. The first method uses a 19-bp sequence of E. coli, GATAATGATAATCATTATC, which may be read in the forward or reverse direction (Calderwood and Mekalanos, 1988). Using this method, a potential Fur box with 8 of 19 nucleotides identical to the Fur consensus sequence in E. coli was identified between the -10 and -35 regions of the secA promoter (Figure 8A). Likewise, a Fur-like sequence with 10 of 19 nucleotides was identified in the sod upstream region (Figure 8A). The second method uses palindromic sequences of $6 \mathrm{bp}, \mathrm{AT}^{\mathrm{A}} / \mathrm{TAT}$ (Escolar et al., 1999). The palindromic method resulted in more consensus with a Fur box in secA and less homology in the promoter region of sod (Figure 8B). The potential Fur boxes found upstream of both secA and sod ORFs are possibly more related to the AT richness of the genome rather than to transcriptional regulation by metal binding.

Development of Fe-limiting culture conditions. To examine the role of Fe concentration as a regulatory factor in the borrelial SOD expression, the growth medium for B. burgdorferi was modified to limit Fe availability. Routinely, Sh-2-82 was cultured in BSK-II supplemented with heat-inactivated 6\% rabbit serum (Table 1). Although Fe compounds are not added directly to the medium, there are many potential sources of Fe including rabbit serum, gelatin, and BSA. Initially, the Fe-specific chelators, 
Figure 8. Identification of Fur-binding boxes. A. Homology with 19-bp Fur consensus sequence of $E$. coli. B. Homology with 6-bp palindromic sequence in Fur binding box of $E$. coli. Units of six bases are underlined and the sequence identity of bases is marked by asterisks. 
A. Homology with 19-bp consensus sequence of E. coli Fur

GATAATGATAATCATTATC

GTTTTAGTATTACATGATA

$* * \quad * \quad * * * * *$

ATTAAAGATATTAAAGTTT
E. coli Fur box

(Calderwood and Mekalanos, 1988)

B. burgdorferi Fur box in secA promoter

B. burgdorferi Fur box in sod

B. Homology with 6-bp palindromic sequence of E. coli Fur

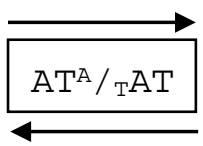

$\frac{\text { GTTTAGTATTACATGATATAAT }}{* * * * * * * *} \frac{\text { TA* }}{* * *}$

$\frac{\text { ATTAAAGATATTAAAGTTTCTAC }}{\star \star \star \star} \frac{\text { ATt }}{\star \star \star \star *} \frac{\text { A }}{\star \star \star *}$
E. coli palindromic Fur box (Escolar et al., 1999)

B. burgdorferi Fur box in secA promoter

B. burgdorferi Fur box in sod promoter 
Desferal and dipyridyl, were tested. With concentrations of 1, 10, and $25 \mathrm{mM}$ of Desferal, no reduction of growth was observed (Figure 9A). This observed lack of effect was unusual since other bacteria like $E$. coli cease to grow in complex media in the presence of $100 \mu \mathrm{M}$ Desferal (Griggs and Konisky, 1989). Similarly, no inhibition of growth was observed with dipyridyl at concentrations of 0.1 to $1 \mathrm{mM}$ (Figure 9B). In $10 \mathrm{mM}$ dipyridyl, there were significant reductions in growth rate and total cell yield (Figure 9C). However, the cells remained greater than 90\% motile like the BSK-II medium control (data not shown). Dipyridyl at final concentrations of $25 \mathrm{mM}$ and $50 \mathrm{mM}$ crystallized out of solution after several days of incubation. Therefore, of the concentrations tested, $10 \mathrm{mM}$ dipyridyl appeared to be sublethal and Fe-restrictive.

The difficulty in restricting Fe by specific chelators led to batch-treatment of BSK-II medium with Chelex-100 resin, which is specific for metal removal in liquids. Iron was added back to the Chelex-treated BSK-II (BSK-II-Clx) medium in the form of ferrous ammonium sulfate $\left[\mathrm{Fe}\left(\mathrm{NH}_{4}\right)_{3}\left(\mathrm{SO}_{4}\right)_{3}\right]$ in Figure 10 or ferric nitrate $(\mathrm{FeNO} 3)$ in Figure 11. In both experiments, cells grew poorly and were nonmotile in BSK-II-Clx with a final density about 10 -fold lower than that observed in the BSK-II control. The addition of $\left[\mathrm{Fe}\left(\mathrm{NH}_{4}\right)_{3}\left(\mathrm{SO}_{4}\right)_{3}\right]$ at final concentrations of $0.1 \mathrm{mM}$ and $1.0 \mathrm{mM}$ was toxic to Sh-2-82 in both chelated and control media (Figure 10). Ferric nitrate did not exhibit the same toxic effects on borrelial cells grown in BSK-II control medium. However, the addition of $\mathrm{FeNO}_{3}$ to BSK-II-Clx resulted in lysis of the culture (Figure 11). It was concluded from these growth curves that the Chelex treatment had removed necessary metals and/or divalent cations from the medium. The addition of free Fe did not replenish the necessary factors for borrelial growth. 
Figure 9. Growth of B. burgdorferi Sh-2-82 in BSK-II medium in the presence of iron chelators. Sh-2-82 was cultured in BSK-II medium supplemented with $6 \%$ heat-inactivated rabbit serum plus Desferal or 2,2'-dipyridyl. A. Desferal, 1 to $25 \mathrm{mM}$. B. Dipyridyl, 0.1 to $1.0 \mathrm{mM}$. C. Dipyridyl, 1.0 to $50 \mathrm{mM}$. Dipyridyl was solubilized in $20 \%$ ethanol and added to BSK-II medium at a final concentration of $0.04 \%$ ethanol. A control for ethanol effects was BSK-II medium without dipyridyl plus 0.04\% ethanol. Cultures were incubated in ambient $\mathrm{O}_{2}$ at $33^{\circ} \mathrm{C}$. Cell density was determined using dark-field microscopy. Values represent ten measurements in two independent experiments. 


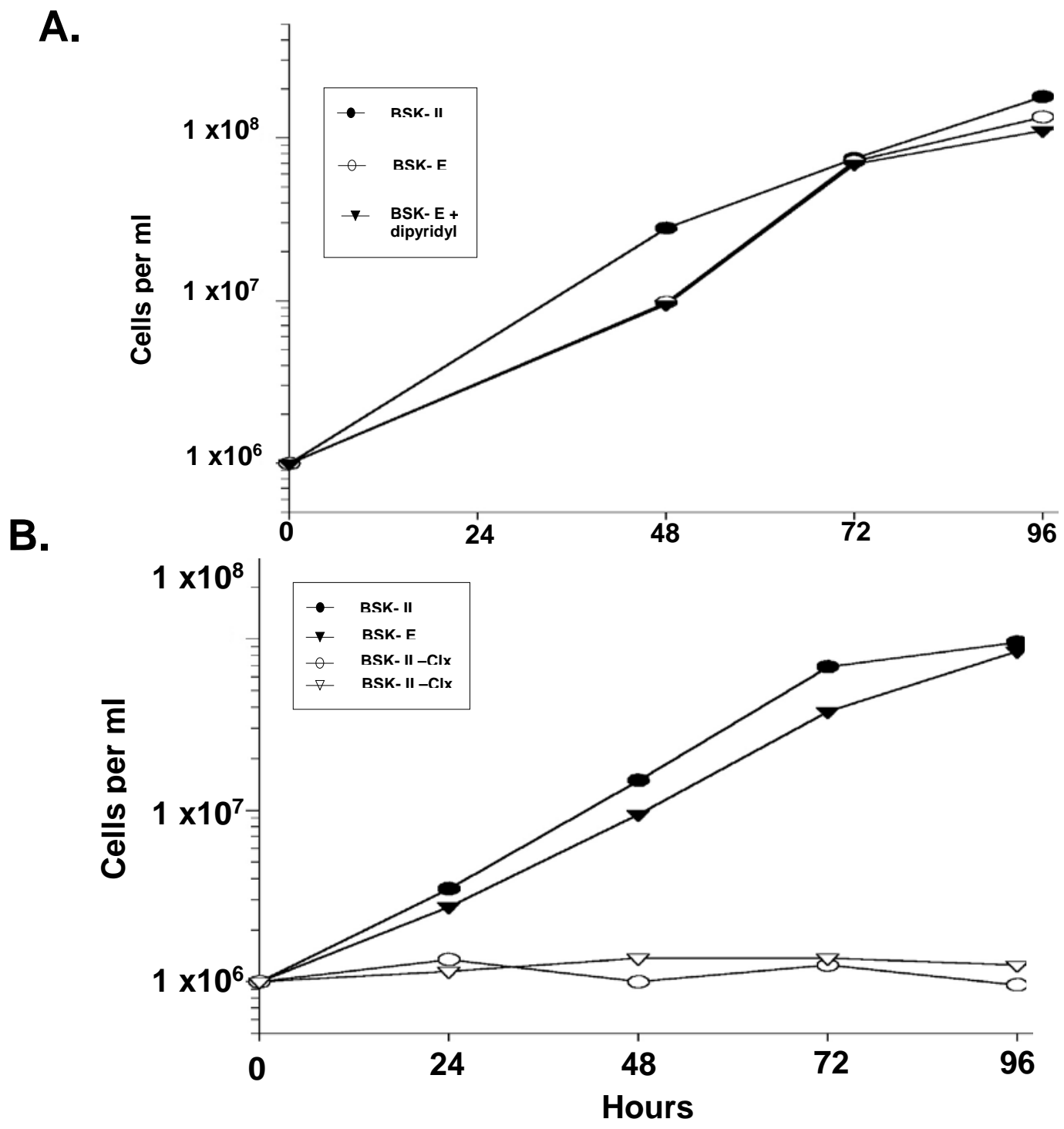


Figure 10. Growth of Sh-2-82 in chelexed BSK-II medium in the presence of ferrous ammonium sulfate. Sh-2-82 was cultured in BSK-II-Clx medium and 6\% heat-inactivated rabbit serum plus $\mathrm{Fe}\left(\mathrm{NH}_{4}\right)_{3}\left(\mathrm{SO}_{4}\right)_{3}$. A concentration range of $0.1 \mathrm{mM}$ to $1.0 \mathrm{mM}$

$\mathrm{Fe}\left(\mathrm{NH}_{4}\right)_{3}\left(\mathrm{SO}_{4}\right)_{3}$ was tested. Cultures were incubated in ambient $\mathrm{O}_{2}$ at $33^{\circ} \mathrm{C}$. Cell density was determined using dark-field microscopy. White symbols, BSK-II treated with Chelex100. Black symbols, BSK-II without Chelex-treatment. Values represent an average of ten measurements in two independent experiments. 


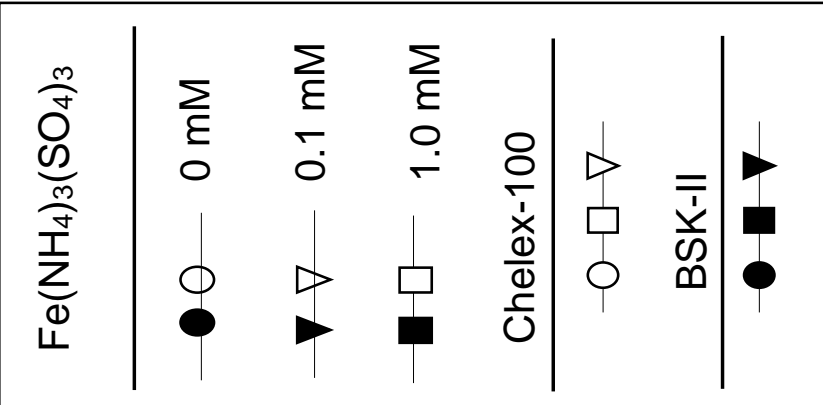

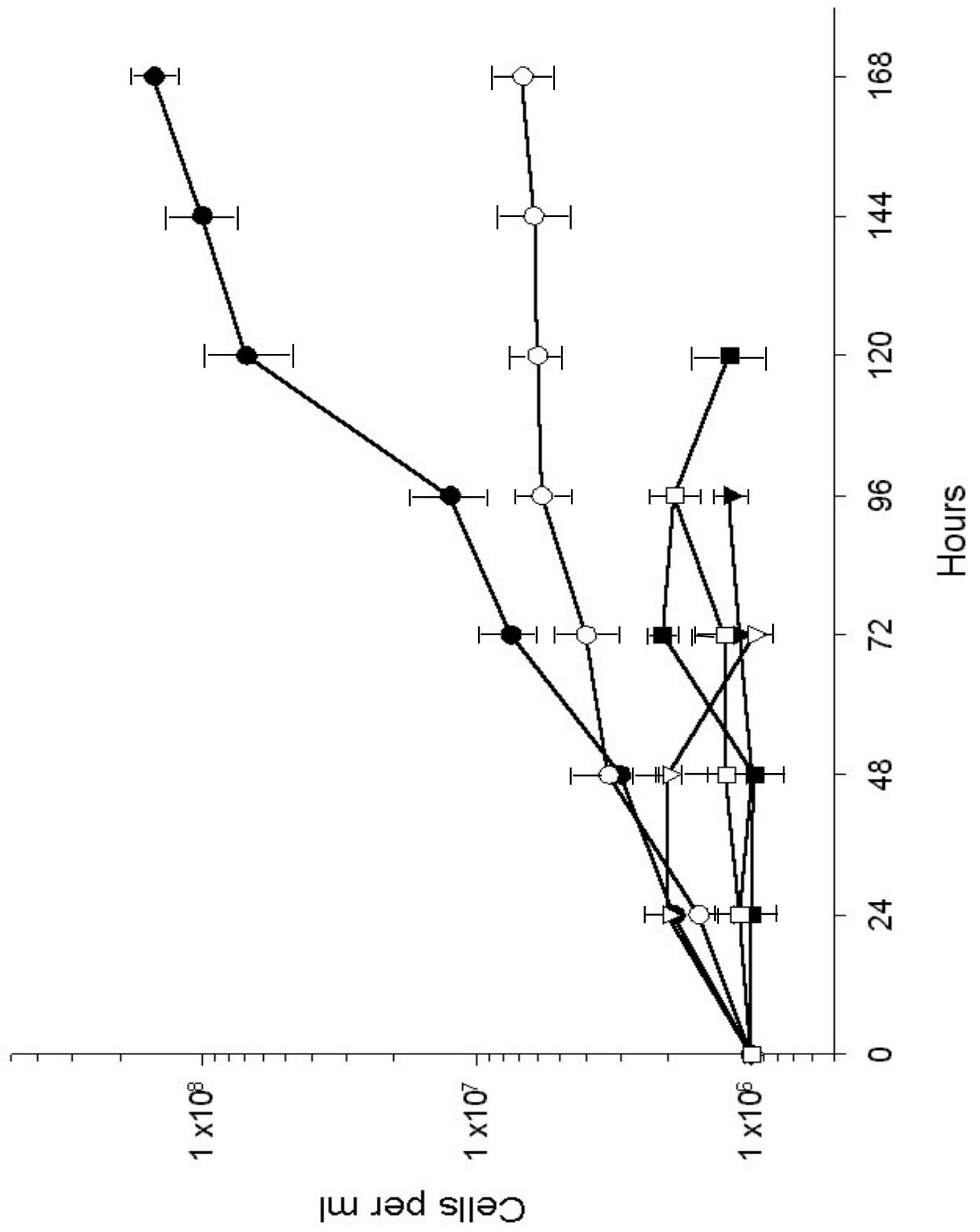


Figure 11. Growth of Sh-2-82 in chelexed BSK-II medium in the presence of ferric nitrate. Sh-2-82 was cultured in BSK-II-Clx medium and 6\% heat-inactivated rabbit serum plus $\mathrm{FeNO}_{3}$. A concentration range of $0.05 \mathrm{mM}$ to $5.0 \mathrm{mM} \mathrm{FeNO}_{3}$ was tested. Cultures were incubated in ambient $\mathrm{O}_{2}$ at $33^{\circ} \mathrm{C}$. Cell density was determined using dark-field microscopy. White symbols, BSK-II-Clx. Black symbols, BSK-II without Chelex-treatment. Values represent an average of ten measurements in two independent experiments. 


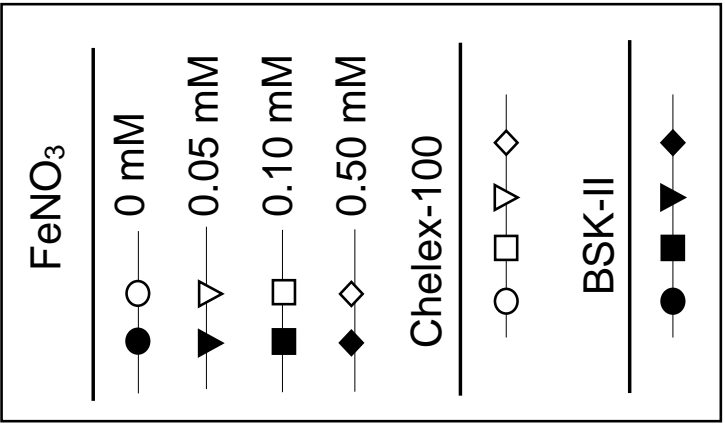

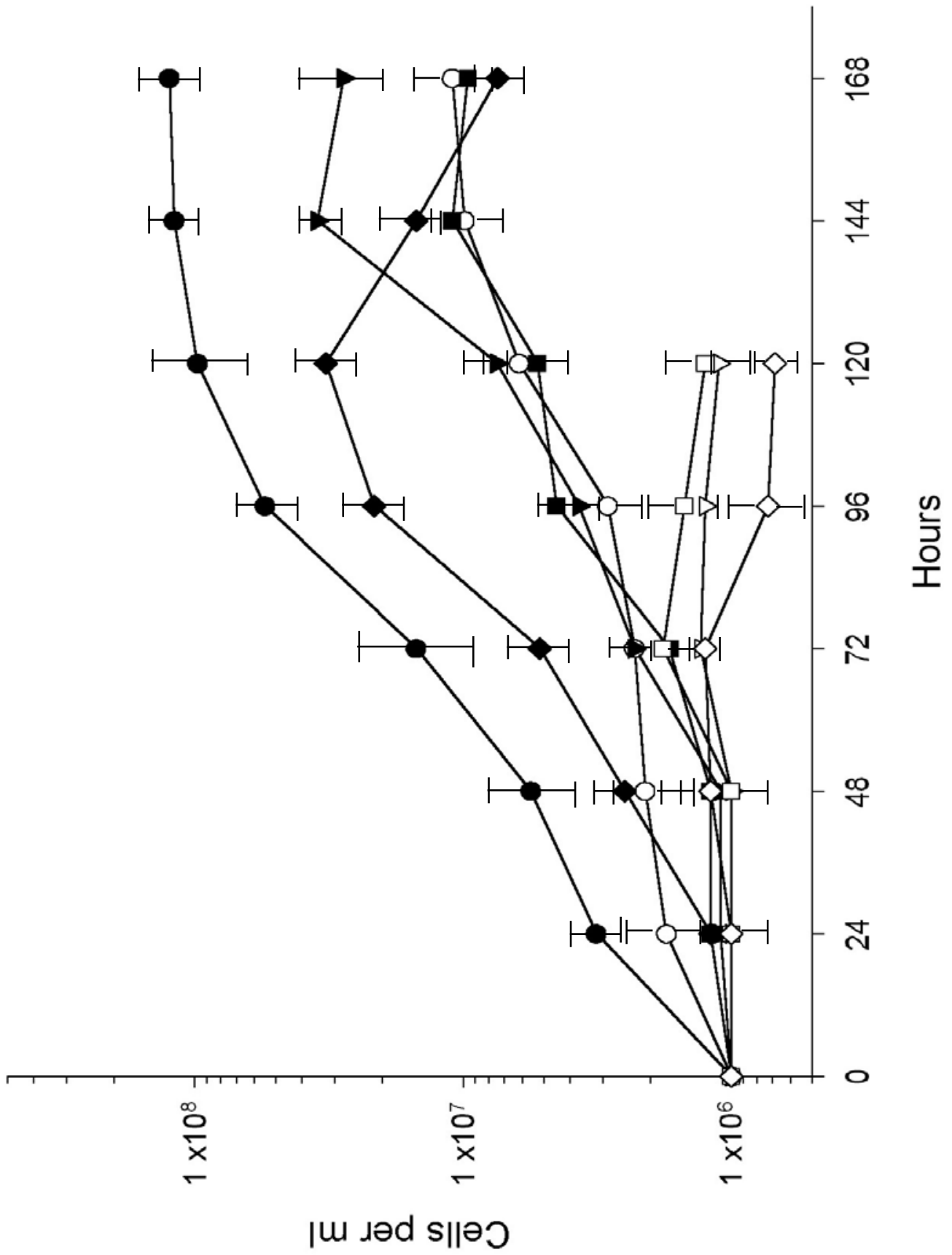


The possibility that alternative methods for obtaining Fe may function in $B$.

burgdorferi was evaluated. B. burgdorferi does not produce siderophores; however, a Tfbinding protein has been identified in B. burgdorferi (Dorward et al., 1994). The next step was to limit Fe by addition of $10 \mathrm{mM}$ dipyridyl and test whether growth of Sh-2-82 was restored by addition of Fe-loaded Tf (holo-Tf). Studies in the uptake and utilization of Fesaturated Tf by Fe-deprived Neisseria showed efficient binding at concentrations of 0.1 to $1.0 \mu \mathrm{M}$ Fe-saturated Tf (McKenna et al., 1988; Pintor et al., 1993). To compensate for the high concentration of dipyridyl needed for Fe-restriction of growth in Sh-2-82, an initial concentration of $3.1 \mu \mathrm{M}$ of holo- or apo-Tf was added. Figures 12A illustrates that both holo- and apo-Tf slowed growth slightly; however, by 96 hours, the Tf-treated cultures had reached stationary phase with the same cell density as that of the BSK-II control. Because the growth was not restored in dipyridyl-treated cultures, the concentration of Tf was increased four-fold (Figures 12B). Neither holo- nor apo-Tf (3.1 $\mu \mathrm{M}$ or $12.5 \mu \mathrm{M})$ restored growth in the presence of $10 \mathrm{mM}$ dipyridyl (Figures 12A, 12B). In addition, no significant growth was observed when Tf was added at 0 hour versus 48 hours of incubation (Figure 12C, 12D). From this set of experiments, it was concluded that Sh-2-82 cells are unable to effectively utilize holo-Tf as an Fe source to enhance growth.

An alternative Fe-restrictive growth medium, BSK-E, was described recently for $B$. burgdorferi (Posey and Gherardini, 2000). It was reported that culturing B. burgdorferi in BSK-E resulted in growth rates and motility similar to those of cells grown in BSK-II medium (Posey and Gherardini, 2000). This finding was confirmed in Figure 
Figure 12. Growth of Sh-2-82 in dipyridyl-treated BSK-II medium in the presence holo-/apo-transferrin. Sh-2-82 was cultured in dipyridyl-treated-BSK-II medium and 6\% heat-inactivated rabbit serum. A. Sh-2-82 cultures +/- $10 \mathrm{mM}$ dipyridyl and $12.5 \mu \mathrm{M}$ Tf. B. Sh-2-82 cultures $+/-10 \mathrm{mM}$ dipyridyl and $3.125 \mu \mathrm{M}$ Tf. C. Comparison of dipyridyl-treated cultures with addition of $12.5 \mu \mathrm{M}$ Tf at 0 or 48 hours of incubation. D. Comparison of dipyridyl-treated cultures with addition of $3.125 \mu \mathrm{M}$ Tf at 0 or 48 hours of incubation. Cultures were incubated in ambient $\mathrm{O}_{2}$ at $33^{\circ} \mathrm{C}$. Cell density was determined using darkfield microscopy. Values represent an average of ten measurements in two independent experiments. 


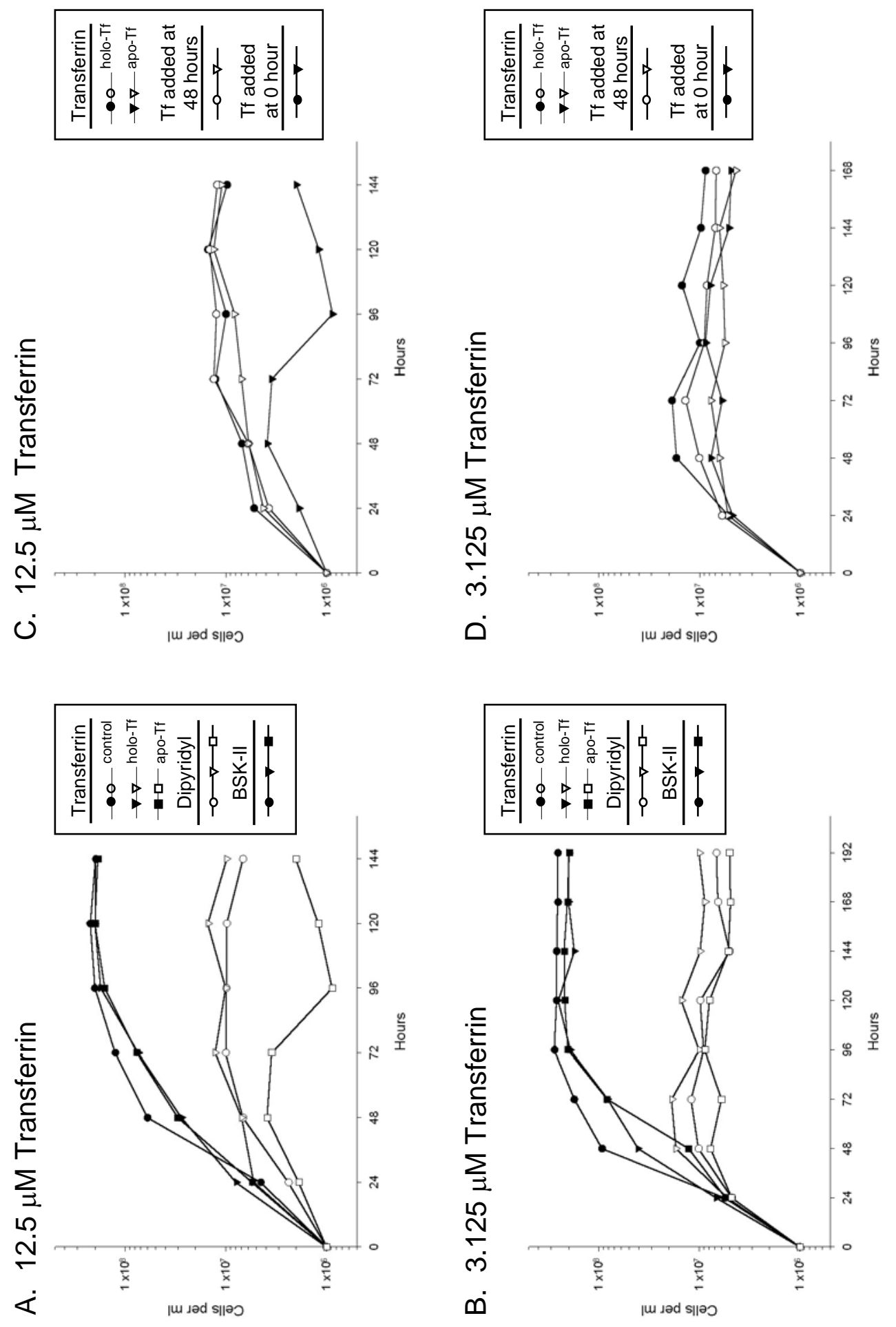


Figure 13. Growth of Sh-2-82 in BSK-Excyte medium. A. Sh-2-82 cultures in BSK-II medium plus $6 \%$ heat-inactivated rabbit serum and BSK-E medium +/- $10 \mathrm{mM}$ dipyridyl. B. Sh-2-82 cultures in BSK-II-Clx and BSK-E-Clx media. C. Sh-2-82 cultures in BSK-E-Clx medium +/- $600 \mu \mathrm{M} \mathrm{MgCl}_{2},+/-10 \mu \mathrm{M} \mathrm{MnCl}_{2},+/-10 \mu \mathrm{M} \mathrm{ZnCl}_{2}$, +/- $10 \mu \mathrm{M} \mathrm{FeCl}_{3}$. Cultures were incubated in ambient $\mathrm{O}_{2}$ at $33^{\circ} \mathrm{C}$. Cell density was determined using dark-field microscopy. 
A.

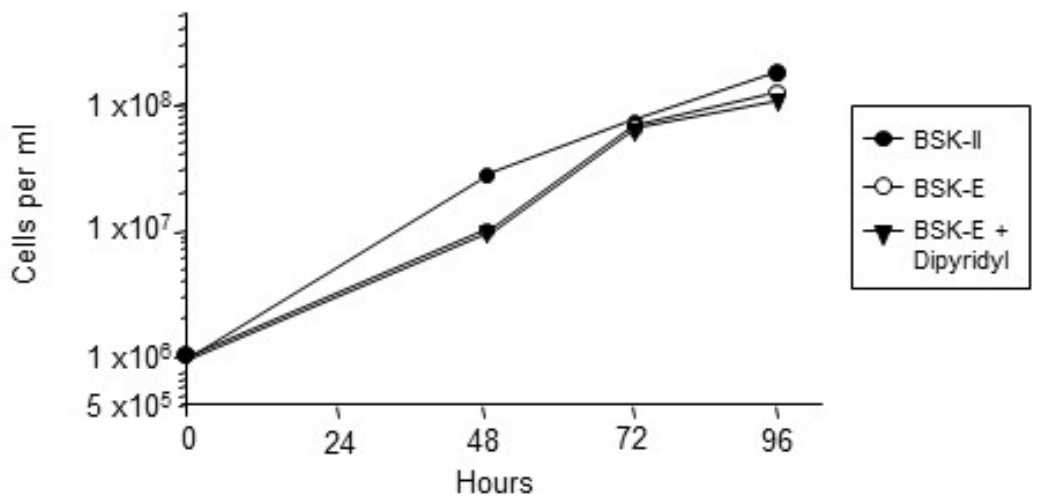

B.

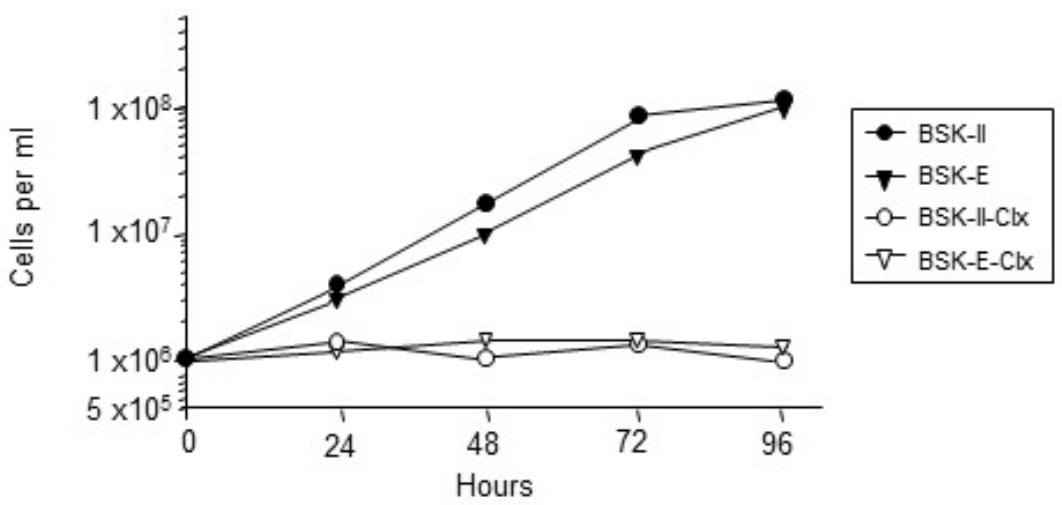

C.

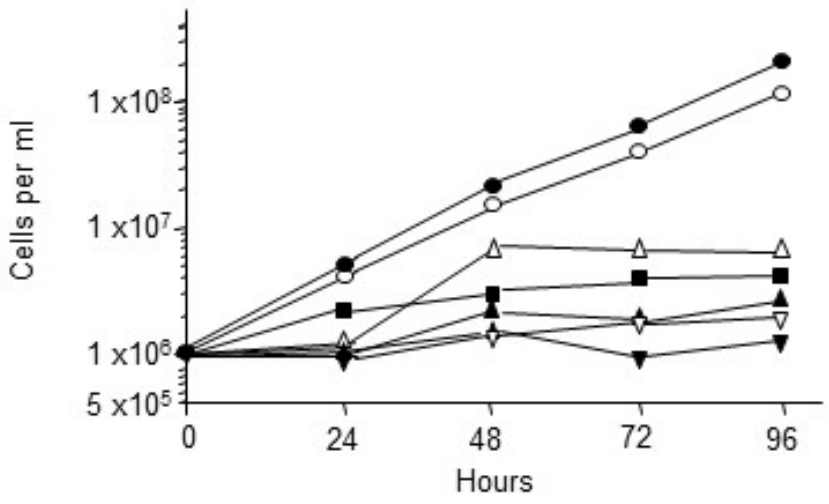

- BSK-E contro SK-E-Clx $=-\mathrm{MgCl}$

$-\Delta+\mathrm{MgCl}_{2}+\mathrm{MnCl}_{2}$

$-\mathbf{A}+\mathrm{MgCl}_{2}+\mathrm{ZnCl}_{2}$

$-\overline{-}-+\mathrm{MgCl}_{2}+\mathrm{MnCl}_{2}+\mathrm{ZnCl}_{2}$

$\boldsymbol{\nabla}+\mathrm{MgCl}_{2}+\mathrm{MnCl}_{2}+\mathrm{FeCl}_{2}$ 
dipyridyl to BSK-E medium did not exhibit growth inhibition of Sh-2-82 as previous experiments using BSK-II medium (Figure 13A). Sh-2-82 failed to grow in both BSK-II and BSK-E when treated with Chelex (Figure 13B). Metals were added individually and in combination back to the medium in order to restore growth as described by Posey and Gherardini (2000). The addition of $600 \mu \mathrm{M} \mathrm{MgCl}_{2}, 10 \mu \mathrm{M} \mathrm{MnCl}_{2}$, and $10 \mu \mathrm{M} \mathrm{ZnCl}_{2}$ restored growth (Figure 13C). Because Mg is required for ribosomal functions, Mg was constant in all cultures. The single addition of $\mathrm{Mn}$ or $\mathrm{Zn}$ did not enhance growth; however, when both $\mathrm{Mn}$ and $\mathrm{Zn}$ were added in combination with $\mathrm{Mg}$, growth was restored. The addition of Fe to BSK-II-Clx showed no growth, which is consistent with the above results (Figures 10 and 11). From these experiments, it was concluded that chlelation had removed metals other than Fe that were critical for growth; furthermore, Mn, Zn, and Mg, but not Fe, are required for growth of $B$. burgdorferi.

The purpose of the above experiments was to identify a condition in which growth of Sh-2-82 was inhibited by restricting Fe availability. As stated previously, the growth medium for $B$. burgdorferi is complex and difficult to manipulate. In the initial investigations with micromolar amounts of Desferal and dipyridyl, inhibition of growth was not consistent with that reported in the literature for other bacteria (Kesavalu et al., 1999; Poole et al., 1996; Reissbrodt et al., 1997). However, growth of Sh-2-82 was inhibited in BSK-II medium plus $10 \mathrm{mM}$ dipyridyl. On the contrary, no major differences were observed in growth of Sh-2-82 in dipyridyl-treated BSK-E as compared 
Figure 14. Effect of manganese on the growth of Sh-2-82. Sh-2-82 was cultured in BSKE-Clx medium supplemented with $600 \mu \mathrm{M} \mathrm{MgCl}_{2}, 10 \mu \mathrm{M} \mathrm{ZnCl}_{2}$, and various concentrations of $\mathrm{MnCl}_{2}$. Cultures were incubated in ambient $\mathrm{O}_{2}$ at $33^{\circ} \mathrm{C}$. Cell density was determined using dark-field microscopy. 


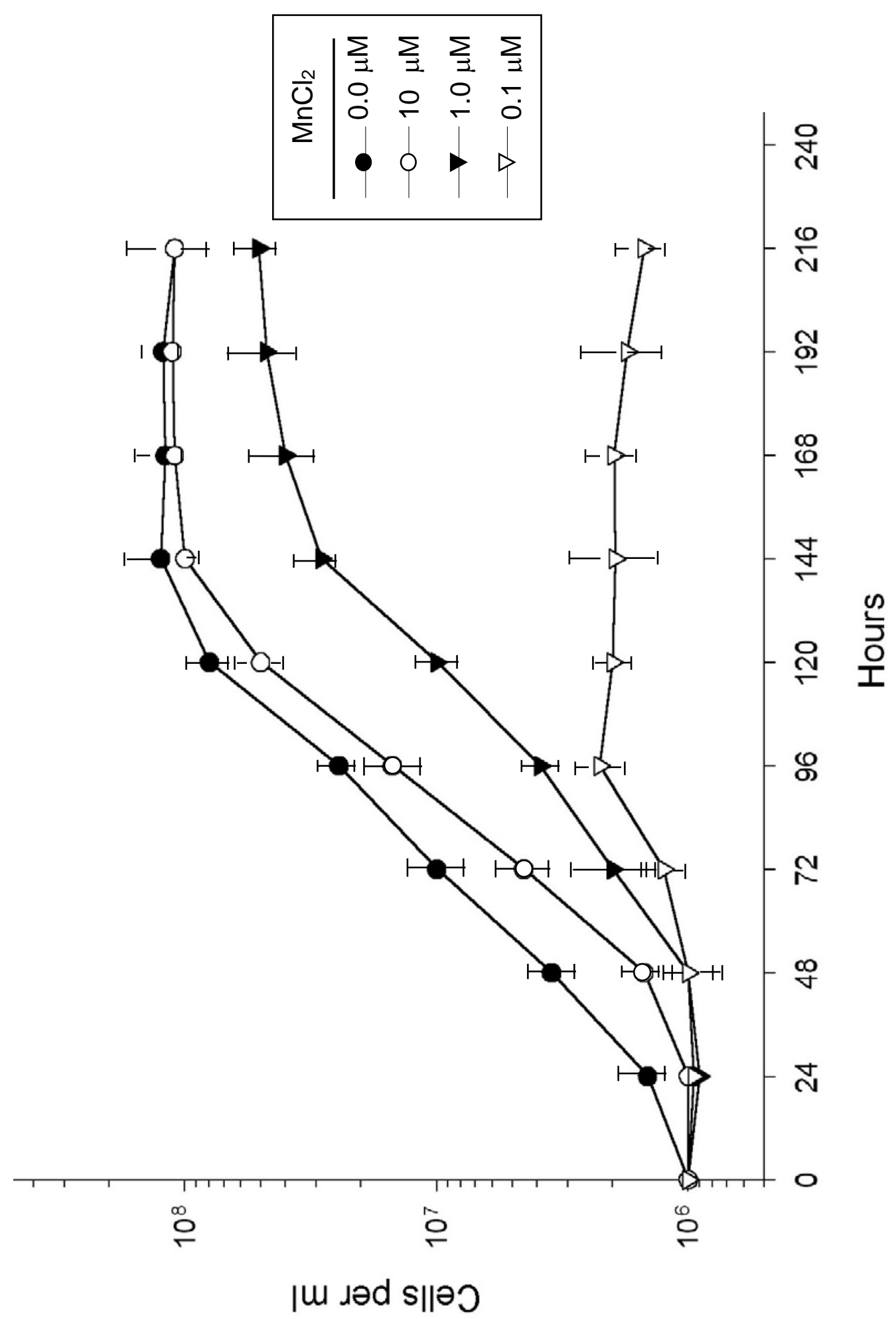


to BSK-E; although, BSK-E plus dipyridyl was considered Fe-restrictive. Differences in growth also were observed in the two methods used in treating the growth medium with Chelex-100. When Sh-2-82 cells were incubated in BSK-II-Clx medium, no growth was observed and cells become irregularly shaped. Likewise, in BSK-E-Clx, Sh-2-82 failed to grow unless Mn, Zn, and Mg were added back. By not adding Fe back, this BSK-E-Clx medium was considered Fe-restrictive.

The observed requirement for $\mathrm{Mn}$ in Figure 13C was interesting, since the $B$. burgdorferi sod closely resembles MnSODs in other bacteria (Fraser et al., 1997). In order to verify the Mn requirement, Mn- limiting growth studies were conducted. Figure 14 shows that the Sh-2-82 culture in BSK-E-Clx supplemented with $1.0 \mu \mathrm{M} \mathrm{MnCl} 2$ grew more slowly than the control culture in BSK-E-Clx. The cell density of the culture supplemented with 1.0 $\mu \mathrm{M} \mathrm{MnCl} 2$ reached approximately $2 \times 10^{7}$ cells per milliliter at 144 hours, whereas the culture supplemented with $10 \mu \mathrm{M} \mathrm{MnCl}_{2}$ reached stationary phase at approximately $1 \times 10^{8}$ cells per milliliter. No significant growth was observed in the culture supplemented with 0.1 $\mu \mathrm{M} \mathrm{MnCl}_{2}$. From this set of growth experiments, BSK-E-Clx supplemented with $600 \mu \mathrm{M}$ $\mathrm{MgCl}_{2}, 10 \mu \mathrm{M} \mathrm{ZnCl}_{2}$, and $1.0 \mu \mathrm{M} \mathrm{MnCl}_{2}$ was established as a Mn-restricted growth condition. 


\section{Chapter 3- Expression of $\sec A$-sod as a function of metal availability}

Development of ribonuclease protection assay. Experiments were conducted to optimize the RPA for use in quantitating mRNA levels of secA and sod in B. burgdorferi. The RPA method has the advantage of a detection level greater than that of Northern analysis and simultaneous detection of multiple transcripts in the same test reaction. Another advantage is that the RPA method is more tolerant of degraded RNA that might hybridize to short oligonucleotide probes. The oligonucleotide probes used in this study are the reverse complement sequence of the gene of interest and are shown in Table 3. The RNA antisense probes for the genes of interest contain a non-homologous tract of 10 adenine nucleotides. After hybridization with target mRNA, this A-tract is cleaved from the full-length probe resulting in a shorter protected fragment that can be resolved by polyacrylamide gel electrophoresis. As a control, flaB message was compared to that of $\sec A$ and $\operatorname{sod}$. The borrelial FlaB protein has been identified in all stages of growth and is excreted via a SecAindependent pathway (Charon et al., 1992). The density of each band was quantified using Quantity One software. The integrated optical density (OD) unit values were normalized to control values of flaB. In short, the OD value for flaB was considered equal to 1.0; then the values for $\sec A$ and sod were divided by the corresponding values of flaB.

Figure 15A shows no difference in migration patterns between individual biotinylated oligonucleotide probes and mixture of probes; therefore, simultaneous detection of $\sec A$, sod, and flaB within the same test tube could be achieved. Efficiency 
Figure 15. Development of RPA. A. Verification of full-length probes, secA, sod, and flaB. Total Sh-2-82 RNA was hybridized to biotinylated oligonucleotide probes and then digested with S1nuclease/RNase enzyme mix (Ambion). The enzyme mix was titrated to determine digestion efficiency and optimal enzyme concentration. B. Determination of probe excess by combining various concentrations of oligo probe mix with various enzyme concentrations. Oligo probe mix: secA plus sod plus flaB. C. Quantitation of RPA. 
A.

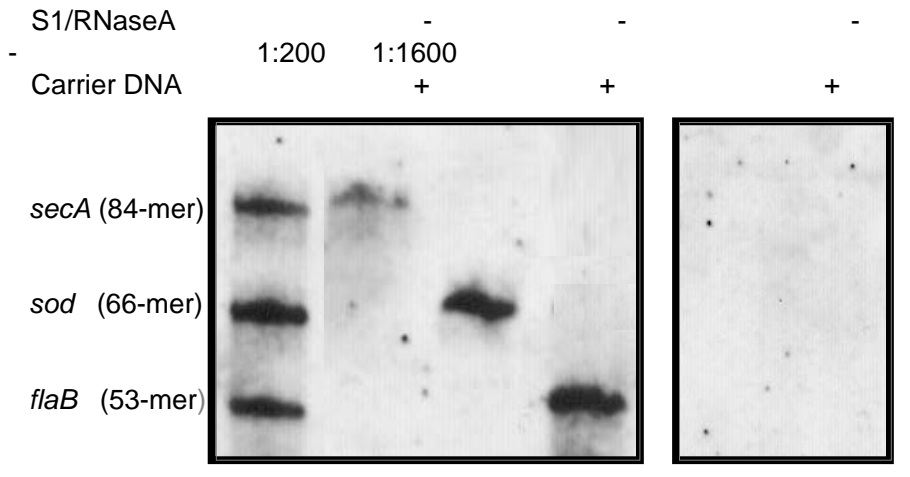

B.

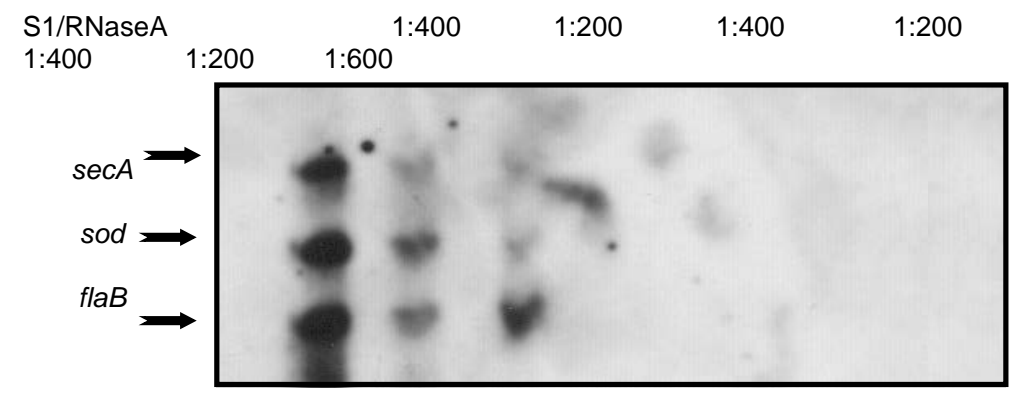


of nuclease digestion was examined by diluting the nuclease mixture, S1/RNaseA, at 1:200

and 1:1600 (Figure 15A). Both nuclease dilutions were effective in digesting probes with no background (Figure 15A). Optimization of nuclease and probe concentrations was done by hybridizing $10 \mu \mathrm{g}$ total RNA with increasing amounts of a mix of $\sec A$, sod, and flaB probes followed by nuclease digestion with one of two nuclease concentrations tested. Optimal probe concentration was observed at 1:100 with efficient digestion at nuclease concentration of 1:200 (Figure 15B). The third pilot experiment was performed to ensure that the RPA was quantitative. As shown in Figure 15C, increasing amounts of total RNA showed increased detection of message. From this set of experiments, the conditions were established for the following transcriptional analyses. Ten micrograms of total RNA was hybridized with 0.1 $\mu \mathrm{g}$ of each gene-specific probe. Non-hybridized RNA and probe was digested with S1/RNase A enzyme mixture at a 1:200 dilution according to manufacturer’s instructions.

Detection of secA-sod mRNA transcript in different phases of growth. The amount of secA-sod message in Sh-2-82 was measured using RPA. Total RNA was extracted from Sh-2-82 cells in different phases of growth. The timepoints represented the following phases of growth: 72 hours, mid-exponential; 96 hours, late-exponential; 120 hours, early stationary; and 144 hours, late stationary (Figure 16A). When hybridized to gene-specific probes, a shift was detected between protected and unprotected fragment lengths (Figure 16B). In comparison to flaB message as an internal control, there was a slight increase in secA and sod message as cells progressed from exponential to stationary phase. The amounts of mRNA protected by the probe were quantified using Quantity One software (BioRad), and the results are shown in Figure 16C. 
Figure 16. Expression of secA-sod transcript as a function of growth phase. A. Sh-2-82 cells was cultured in BSK-II medium supplemented with 6\% heat-inactivated rabbit serum. Arrows indicate the time points at which cells were harvested for RPA analysis. B. RPA analysis of B. burgdorferi secA-sod mRNA in different phases of growth. Biotinylated oligonucleotide probes to secA, sod, and flaB were hybridized with $10 \mu \mathrm{g}$ total RNA. Fulllength oligonucleotide probe sizes are indicated to the left of the representative bands and protected probe fragment sizes to the right. Lane U, undigested full-length probes. Lane D, S1 nuclease and RNase digested probes. Lanes 3-6 represent the protected fragments in RNA isolated from 72, 96, 120, and 144 hour cultures. C. Densitometric analysis of gel bands described above using Quantity One software. The values represent an average of three experiments. Densitometric values are based on the number of pixels in each band. The $\sec A(\mathbf{\square})$ and $\operatorname{sod}(\square)$ values were normalized to flaB values (OD value of secA/OD value of flaB; OD value of sod/OD value of flaB). Values are an average of three experiments. Pixel values of flaB are as follows: 72 hours, 207,293 \pm 15,872; 96 hours, 245,656 \pm 26,893; 120 hours, 233,789 \pm 11,673; 144 hours, 232,681 $\pm 19,405$. 
A.

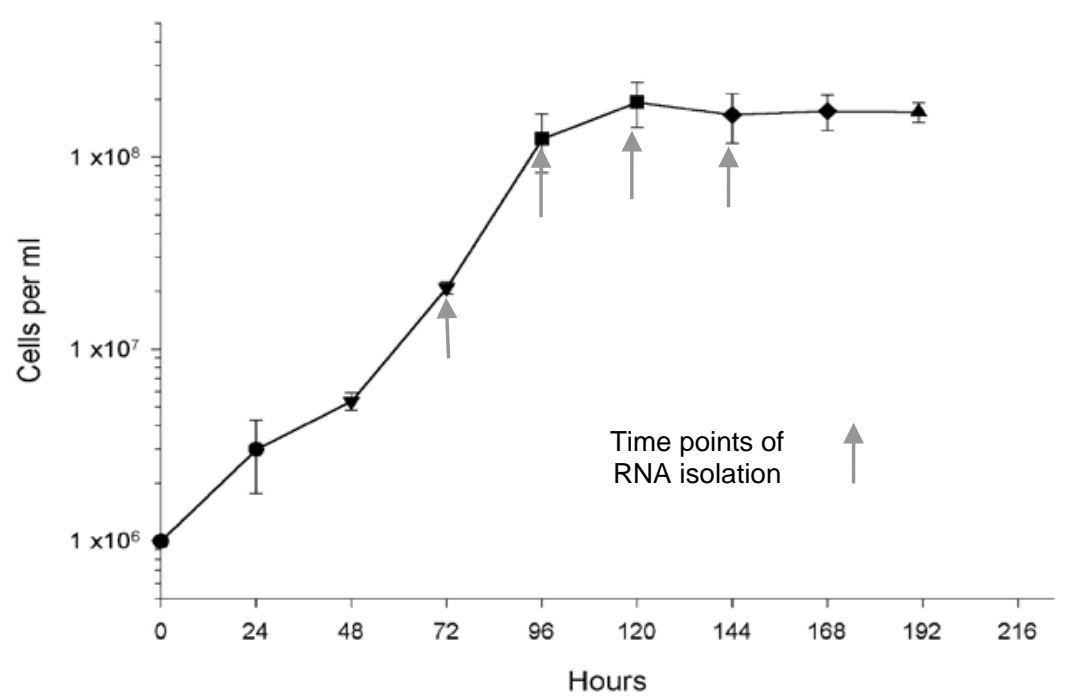

B.

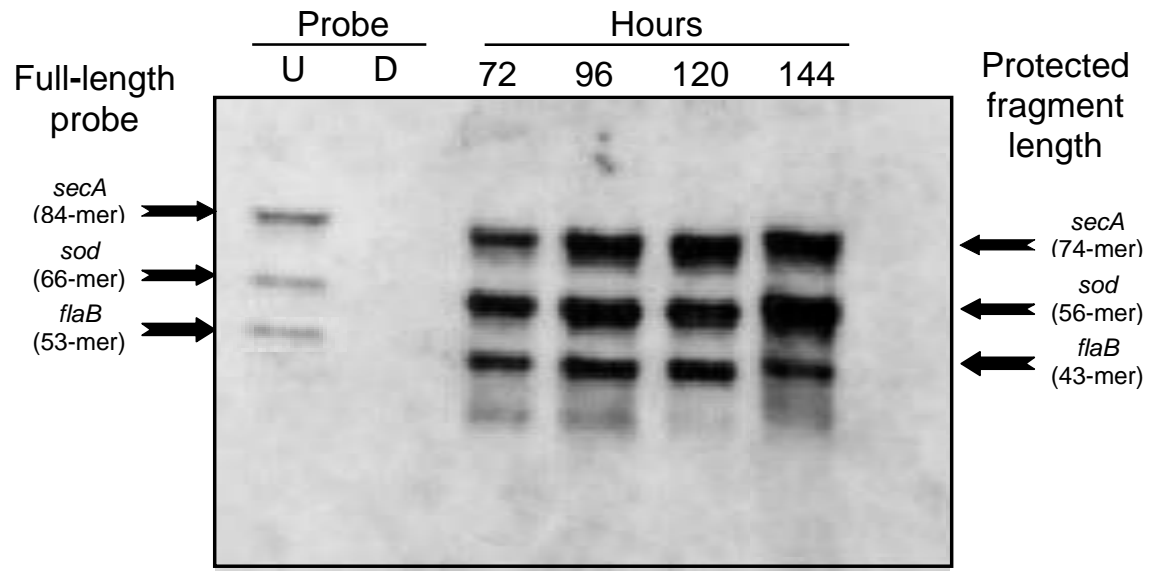

c.

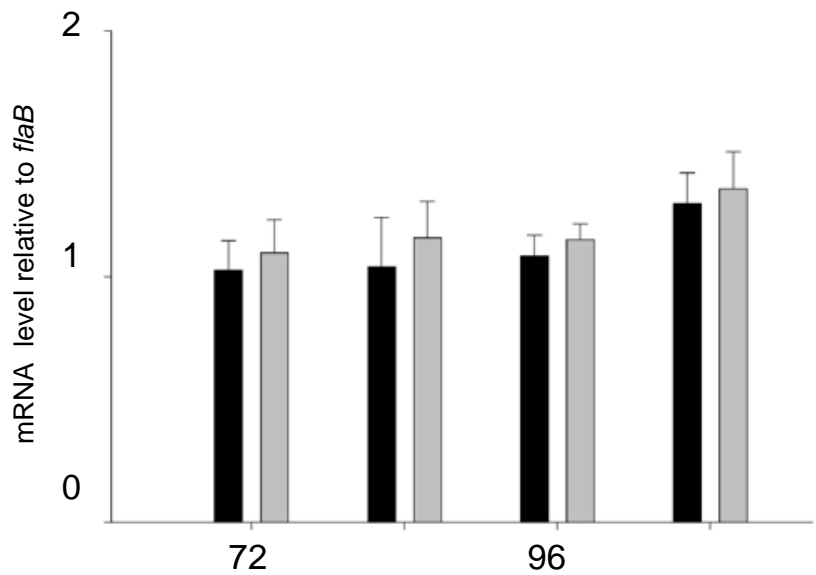


The amounts of protected secA and sod products were normalized to the amount of protected flaB product. The highest yield of secA and sod message was in late stationary phase at 144 hours.

Detection of $\sec A$-sod mRNA transcript as a function of metal availability. From the growth studies in Chapter 2, it was determined that the following growth conditions would provide specific metal restrictions. They are: BSK-E + $10 \mathrm{mM}$ dipyridyl for Fe restriction; BSK-E + 10 mM dipyridyl + $10 \mu \mathrm{M} \mathrm{MnCl}_{2}$ for Fe-restriction and Mnenrichment; BSK-E-Clx + $1.0 \mu \mathrm{M} \mathrm{MnCl}_{2}$ for Fe- and Mn-restriction; BSK-E-Clx $+10 \mu \mathrm{M}$ $\mathrm{MnCl}_{2}$ for Mn-enrichment. B. burgdorferi Sh-2-82 cells were cultured in BSK-E medium with varying amounts of Fe and Mn. Figure 17 shows the growth curve resulting from restriction of Fe and Mn. There was no significant difference between growth of Sh-2-82 in BSK-II medium versus BSK-E medium $(\mathrm{P}<0.1)$. Iron restriction by dipyridyl did not affect growth significantly. In addition, no significant increase in growth was observed by supplementing the dipyridyl-treated medium with $10 \mu \mathrm{M} \mathrm{MnCl}$. The Chelex-100 treated medium was reconstituted with $600 \mu \mathrm{M} \mathrm{MgCl} 2$ and $10 \mu \mathrm{M} \mathrm{ZnCl}$. Manganese chloride was added at either $1 \mu \mathrm{M}$ or $10 \mu \mathrm{M}$ concentrations. Cultures grown in $1 \mu \mathrm{M} \mathrm{MnCl}_{2}$ exhibited an extended lag period. Likewise, the generation or doubling time was lengthened by several hours as compared to that of their growth conditions (Table 4). Overall, motility decreased as hours of incubation increased (Table 4). Sh-2-82 exhibited less motility in media treated with either dipyridyl or Chelex-100 resin. Media enriched with Mn resulted in a higher percentage of Sh-2-82 cells exhibiting motility during exponential phase than media restricted of $\mathrm{Mn}$. 
Figure 17. Growth of Sh-2-82 in metal-defined media. Sh-2-82 was cultured in Ferestrictive medium (either BSK-E plus $10 \mathrm{mM}$ dipyridyl), Fe- and Mn-restrictive medium (BSK-E-Clx plus $1 \mu \mathrm{M} \mathrm{MnCl}_{2}$ ), Fe-restrictive, Mn-enriched medium (BSK-E plus $10 \mathrm{mM}$ dipyridyl plus $10 \mu \mathrm{M} \mathrm{MnCl}_{2}$ or BSK-E-Clx plus $10 \mu \mathrm{M} \mathrm{MnCl}_{2}$ ). The arrows indicate the timepoint at which cells were harvested for RNA isolation. The asterisk indicates the P value $(<0.1)$ of cells cultured in BSK-II versus BSK-E. 


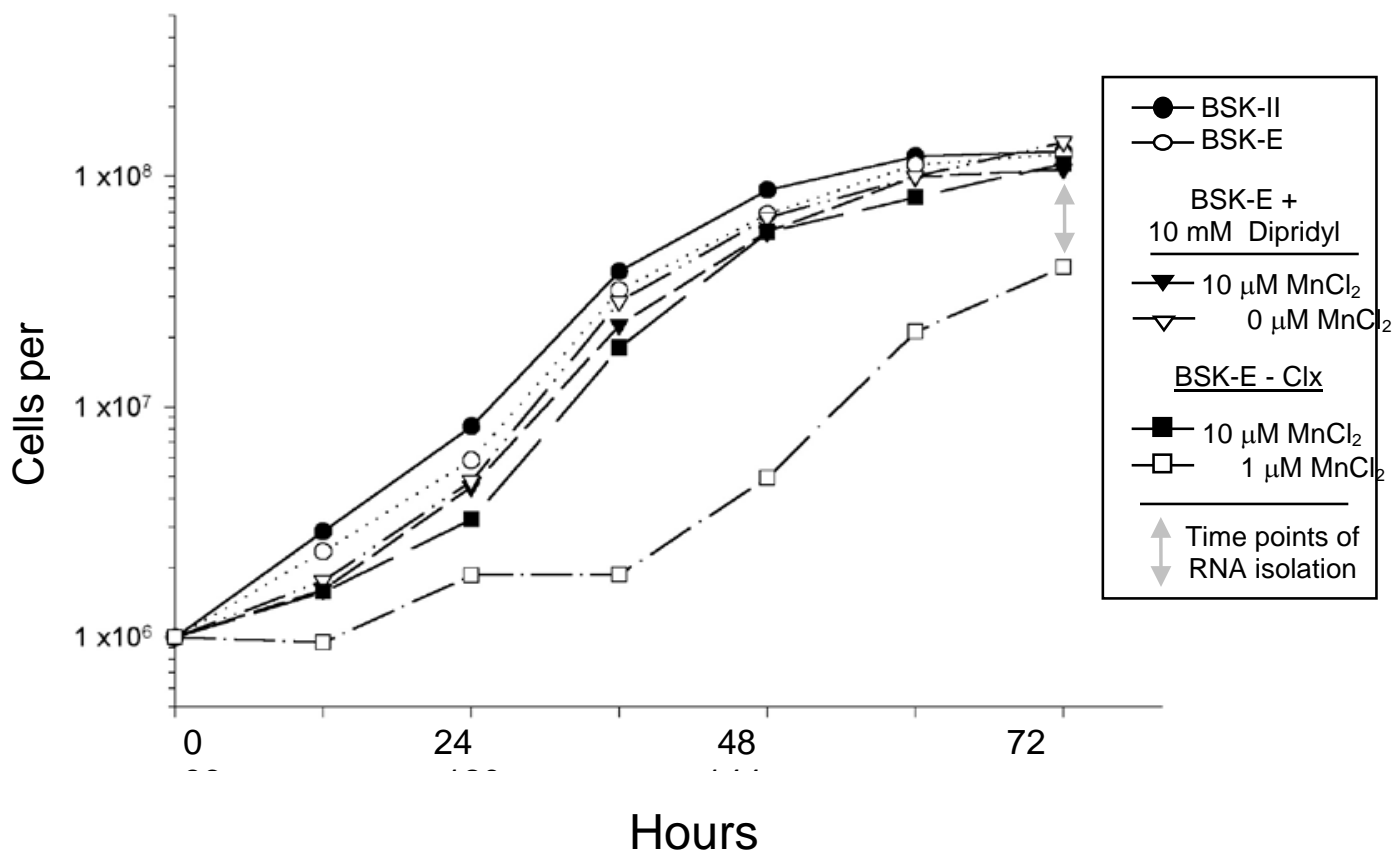




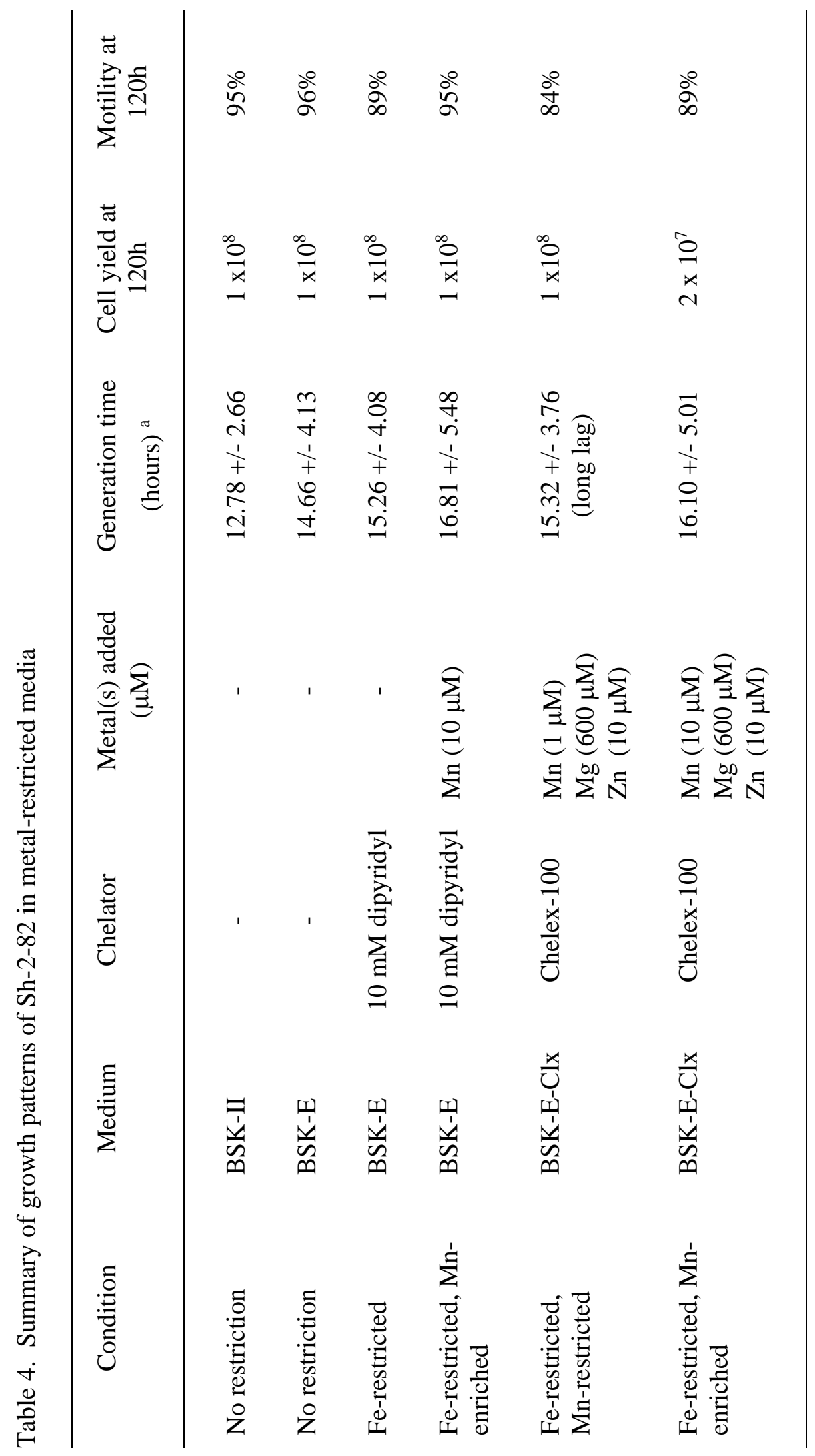


Total borrelial RNA was extracted from Sh-2-82 cells cultured in varying amounts of Mn and Fe (Figure 18A). Ten micrograms of RNA from each culture condition was hybridized to gene specific probes to assess relative abundance of $\sec A$ and $\operatorname{sod}$ mRNA. As a control, the expression of flaB was compared to secA and sod expression. There was no significant induction or repression of secA or sod expression in any of the growth conditions tested (Figure 18C). A reduction in overall transcription was observed in BSK-E-Clx cultures despite the reconstitution with $\mathrm{Mg}, \mathrm{Mn}$, and $\mathrm{Zn}$. The integrity of the RNA extracted from the BSK-E-Clx was comparable to that of the other culture conditions (Figure 18A). However, in all culture conditions tested, the expression of sod remained higher than that of $\sec A$. The explanation for a higher expression of sod is unclear since sod is the second gene in this bicistronic message from which transcription is initiated upstream of $\sec A$. 
Figure 18. Expression of $\sec A$-sod transcript as a function of metal availability. A. Total borrelial RNA was extracted from cultures varying in metal composition at 144 hours of incubation as shown in Figure 17. Integrity and quantity of RNA was determined by measuring absorbance at $\lambda 260$ and $\lambda 280$. B. RPA analysis of B. burgdorferi secA-sod mRNA as a function of $\mathrm{Mn}$ and Fe availability. Biotinylated oligonucleotide probes to secA, sod, and flaB were hybridized with $10 \mu \mathrm{g} B$. burgdorferi total RNA. Full-length oligonucleotide probe sizes are indicated to the left of the representative bands and protected probe fragment sizes to the right. Lane U, undigested full-length probes. Lane D, S1 nuclease and RNase digested probes. Lanes 3-8 represent the protected fragments in RNA isolated from the different metal conditions as described in the text. C. Densitometric analysis of gel bands described above using Quantity One software. Densitometric values are based on the number of pixels in each band. The secA (black bars) and sod (gray bars) values were normalized to flaB values. Pixel values of flaB are as follows: BSK-II, 195,952 \pm 23,861; BSK-E, 207,441 + 35,853; BSK-E plus dipyridyl, 210,649 + 16,693; BSK-E plus dipyridyl plus $10 \mu \mathrm{M} \mathrm{MnCl}_{2}, 172,383 \pm 13,214$; BSK-E-Clx plus $1 \mu \mathrm{M} \mathrm{MnCl}_{2}, 140,761 \pm$ 15,113; BSK-E-Clx plus $1 \mu \mathrm{M} \mathrm{MnCl}_{2}, 97,021 \pm 15,722$. Values are an average of three experiments. 
A.

Total RNA Isolated

\begin{tabular}{lcc}
\hline Condition & $\begin{array}{c}\lambda 260 / 280 \\
\text { ratio }^{\mathrm{a}}\end{array}$ & $\begin{array}{c}\text { Concentration } \\
(\mu \mathrm{g} / \mathrm{ul})^{\mathrm{b}}\end{array}$ \\
\hline BSK-II & 1.91 & $2.98 \pm 0.08$ \\
BSK-E & 1.92 & $1.59 \pm 0.24$ \\
BSK-E+ Dipyridyl & 1.63 & $0.91 \pm 0.30$ \\
BSK-E+ Dipyridyl $+10 \mu \mathrm{M} \mathrm{MnCl}_{2}{ }^{\mathrm{c}}$ & 1.71 & $1.87 \pm 0.50$ \\
BSK-E-Clx $+10 \mu \mathrm{M} \mathrm{MnCl}{ }^{\mathrm{c}}$ & 1.78 & $1.44 \pm 0.88$ \\
$\mathrm{BSK}^{\mathrm{E}} \mathrm{E}-\mathrm{Clx}+1 \mu \mathrm{M} \mathrm{MnCl}{ }^{\mathrm{c}}$ & 1.82 & $1.20 \pm 0.62$ \\
\hline
\end{tabular}

a $\lambda 260 / 280$ ratio of RNA should be $1.8-2.0$ for acceptable purity

${ }^{b}$ (absorbance at $\left.\lambda 260\right) \times$ (dilution factor) $\times 40$

${ }^{c}$ BSK-E-Clx was also supplemented with $600 \mu \mathrm{M} \mathrm{MgCl}_{2}$ and $10 \mu \mathrm{M} \mathrm{ZnCl}_{2}$

B.

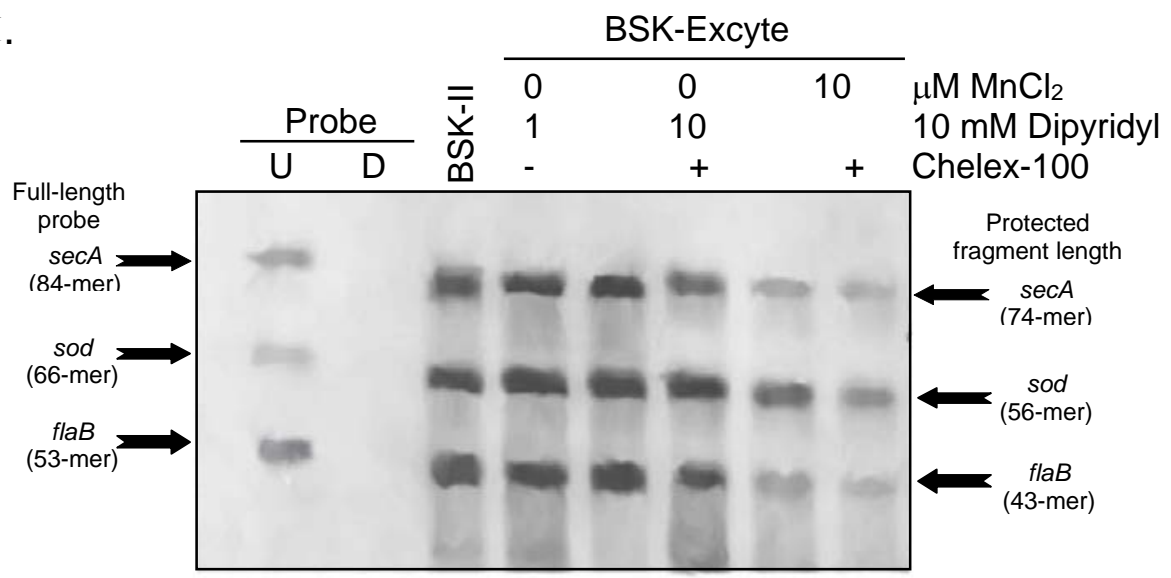

C.

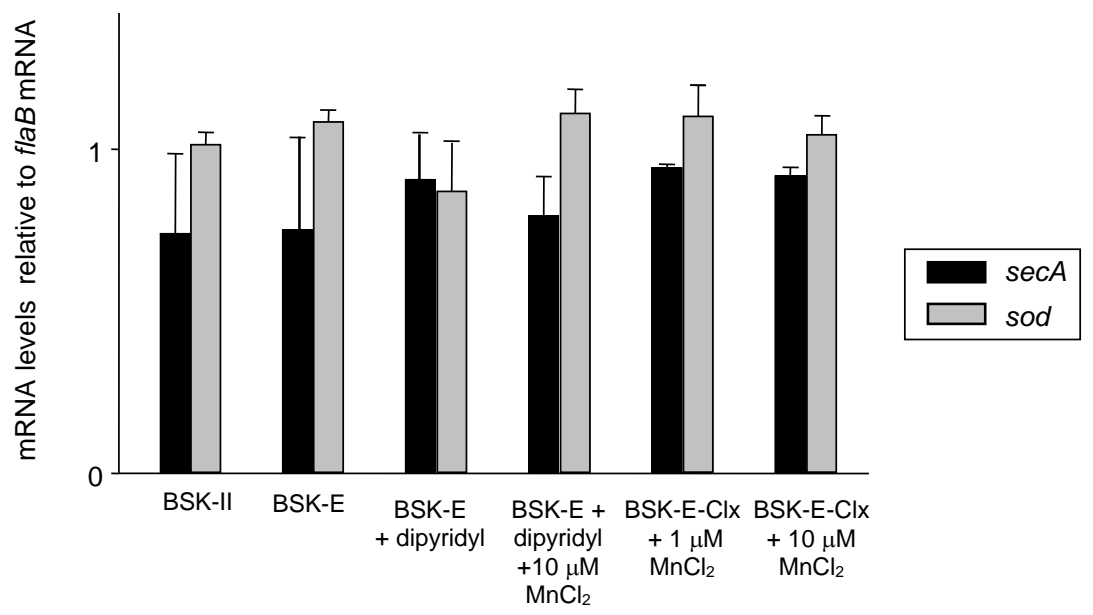




\section{Chapter 4 - Protein profiling of B. burgdorferi Sh-2-82}

Demonstration of SOD activity. The effects of Fe- and Mn-restriction and Mnenrichment on expression of SOD activity were analyzed on native PAGE gels (Davis, 1964; Beauchamp and Fridovich, 1971). As expected, E. coli EMG-2 exhibited three achromatic bands, which correlated with previously described bands of SOD activity (Figure 19). The faster migrating bands $\left(\mathrm{R}_{\mathrm{f}}=0.43\right.$ and $\left.\mathrm{R}_{\mathrm{f}}=0.59\right)$ were inactivated by $\mathrm{H}_{2} \mathrm{O}_{2}$, which is indicative of FeSOD. The slowest migrating band $\left(\mathrm{R}_{\mathrm{f}}=0.29\right)$ did not exhibit sensitivity to $\mathrm{H}_{2} \mathrm{O}_{2}$ or NaCN, indicating that this band was a MnSOD. The SOD activity in the borrelial CFEs was visualized in gels as a single achromatic band $\left(\mathrm{R}_{\mathrm{f}}=0.42\right)$. As previously described (Whitehouse et al.,1997), a faint band of activity was detected in CFE cultured in BSK-II medium; whereas, no band of activity was visualized in the lane containing CFE from BSKE cultured cells. Likewise, no achromatic zones were detected in Sh-2-82 cells cultured in Fe- or Mn-restricted media. An increase in SOD activity was observed in Mn-enriched medium containing $100 \mu \mathrm{M} \mathrm{MnCl}_{2}$. When assayed in the presence of $\mathrm{H}_{2} \mathrm{O}_{2}$, a known inhibitor of FeSOD, the borrelial bands seen in Mn-enriched medium were unaffected, indicating Mn as the enzyme cofactor. On the contrary, the band of SOD activity visualized in CFE from BSK-II cultured cells disappeared in the presence of $\mathrm{H}_{2} \mathrm{O}_{2}$, which is consistent with earlier findings by this laboratory (Whitehouse et al., 1997). The explanation for the utilization of different metal cofactors, Fe and $\mathrm{Mn}$, in cells grown in different media formulations 
Figure 19. SOD activity of $B$. burgdorferi Sh-2-82 in response to metal availability. Borreliae were cultured in varying concentrations of metals as described in text. Stationary phase cells were lysed by sonication. Four hundred micrograms of borrelial CFE protein were loaded onto each lane and $50 \mu \mathrm{g}$ of E. coli EMG-2 CFE were added to the control lane. Following native PAGE, the gels were stained for SOD activity in the presence of $0.1 \mathrm{mM}$ EDTA with no inhibitor (A.), $5.0 \mathrm{mM} \mathrm{H}_{2} \mathrm{O}_{2}$ (B.), or $1.0 \mathrm{mM} \mathrm{NaCN}$ (C.). Lane 1, BSK-II; Lane 2, BSK-Excyte; Lane 3, BSK-E plus $10 \mathrm{mM}$ dipyridyl culture; Lane 4, BSK-E plus $1 \mu \mathrm{M} \mathrm{MnCl}_{2}$; Lane 5, BSK-E plus $10 \mu \mathrm{M} \mathrm{MnCl}$; Lane 6, BSK-E plus $100 \mu \mathrm{M} \mathrm{MnCl}_{2}$; Lane 7, E. coli EMG-2. 


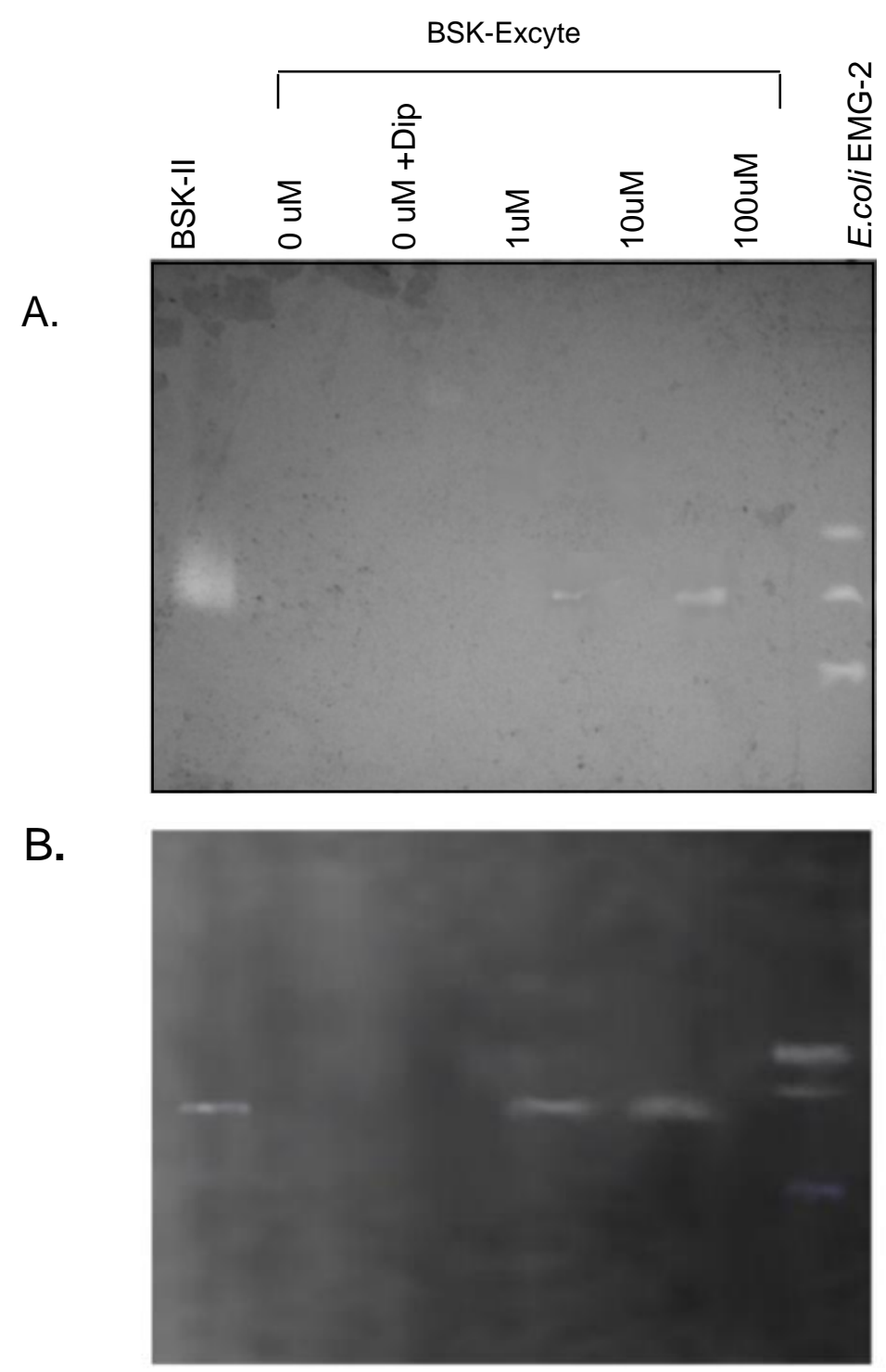

C.

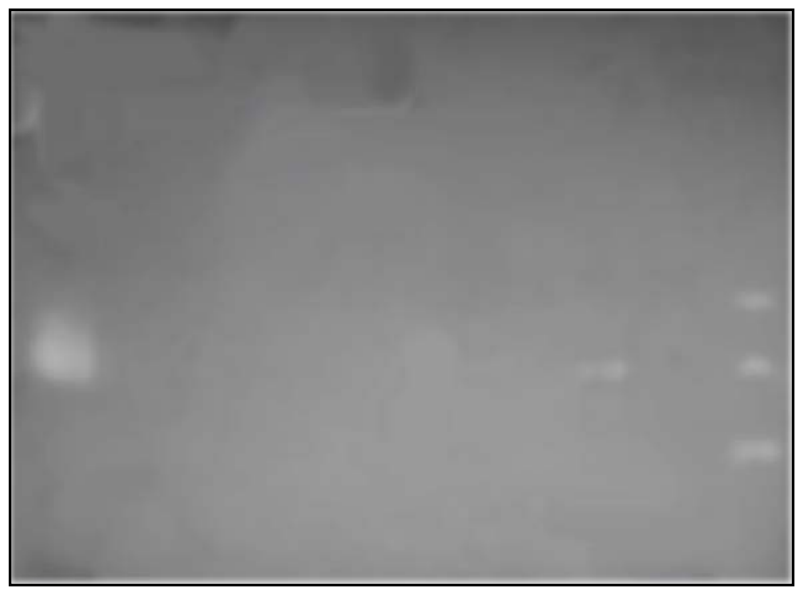


suggests this enzyme maybe a cambialistic SOD.

The presence of SOD activity in Sh-2-82 was analyzed spectrophotometrically by the xanthine oxidase-cytochrome $c$ method of McCord and Fridovich (1969). Borrelial extracts were prepared from cells cultured in different metal concentrations to stationary phase and were assayed for SOD activity. No SOD activity was detected in the borrelial cells grown in BSK-E medium. The maximum amount of CFE protein tested in the assay was $400 \mu \mathrm{g}$. However, an increase in activity was observed as the Mn concentration was increased in the growth medium. When the concentration of $\mathrm{MnCl}_{2}$ was increased from $10 \mu \mathrm{M}$ to $100 \mu \mathrm{M}$, SOD activity in cells increased from $7.3 \pm 2.1$ to $22.7 \pm 6.4$ units/mg of CFE protein. The SOD activity of Sh-2-82 cultured in BSK-II medium was low (12.5 \pm 1.9 units/mg), but was comparable to that described previously (Whitehouse et al., 1997; Smith, 1998). The control E. coli EMG-2 cells cultured aerobically exhibited greater SOD activity at $158.3 \pm 9.5$ units/mg. It is unclear why there was a difference in SOD activity of cells cultured in BSK-II as compared to BSK-E. Unfortunately, Fe-restricted conditions using $10 \mathrm{mM}$ dipyridyl were not tested for SOD activity in the spectrophotometric assay. However, these results, along with the SOD activity gels, suggest that Mn participates in SOD activity of B. burgdorferi when cultured in BSK-E medium.

Effect of metal availability on protein profiles by SDS-PAGE. To identify differences in protein expression, CFEs from stationary phase cells cultured in described media formulations were electrophoresed and stained with Coomassie blue, followed by silver staining. Molecular weights of prominent bands were estimated to be 66, 62, 58, 
Figure 20. Effect of metal availability on the protein profile of $B$. burgdorferi Sh-2-82. Twenty micrograms of borrelial protein was loaded onto each lane and resolved by SDSPAGE. The gel was stained with Coomassie blue, (A), followed by silver staining, (B) Lane 1, BSK-E; Lane 2, BSK-E plus $10 \mathrm{mM}$ dipyridyl; Lane 3, BSK-E plus $1 \mu \mathrm{M} \mathrm{MnCl}$; Lane 4, BSK-E plus $10 \mu \mathrm{M} \mathrm{MnCl}_{2}$; Lane 5, BSK-E plus $100 \mu \mathrm{M} \mathrm{MnCl}_{2}$. 


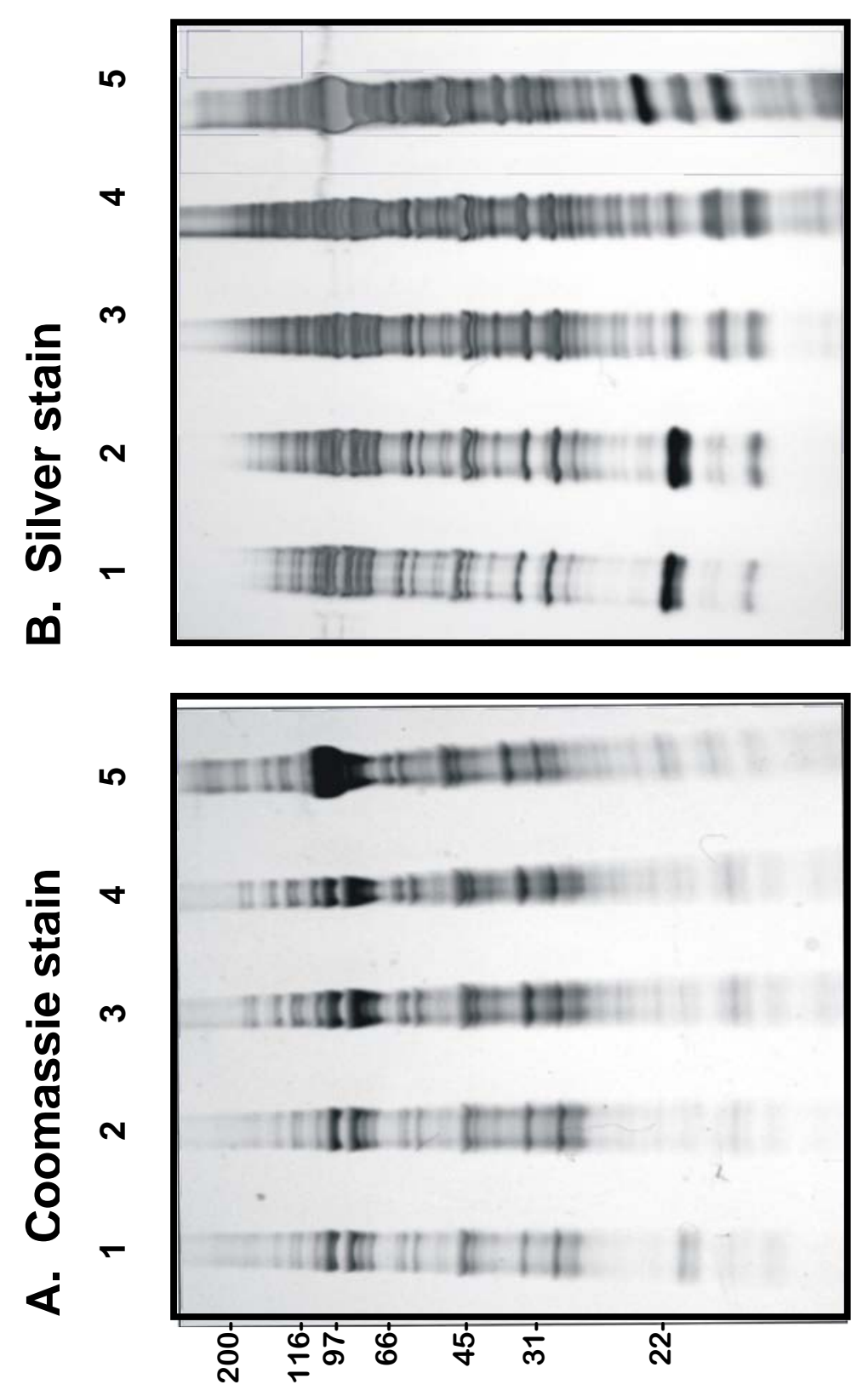


47, 41, 31, 29, 21, $19 \mathrm{kDa}$ (Figure 20). Borrelial proteins that have corresponding molecular weights have been described in the literature (Engstrom et al., 1995; Dressler et al., 1993; Carriero et al., 1990; Cluss and Boothby, 1990). There was increased expression of a band at approximately $14 \mathrm{kDa}$ in the Fe-restricted medium (Figure 20, lane 2). Increasing Mn concentrations resulted also in the induction of two additional bands at 17$18 \mathrm{kDa}$ (Figure 20, lane 5). A band at approximately $21 \mathrm{kDa}$ appeared repressed in low $\mathrm{Mn}$ concentrations (Figure 20, lanes 3 and 4). Between 97 kDa and 116 kDa (Figure 20, lane 5), there was a zone of intense staining resulting from increased expression of one or more proteins. A small spot in the middle of the band area was excised from the gel and processed for peptide mass identification. Identification by MALDI-TOF is based on matching tryptic peptide fragments of a protein to a database containing protein data of sequenced genomes. MALDI-TOF identified several borrelial proteins from the excised gel band analysis using the TIGR database (www.tigr.com) to correlate peptide masses with estimated masses from the genome sequence. In decreasing order of matching data, the proteins were identified as transcription elongation factor (BB132, $105.3 \mathrm{kDa}$ ), chemotaxis histidine kinase (BB669, 98.3 kDa), hypothetical protein (BB326, 107.5 kDa), and DNA mismatch repair protein (BB797, $99.8 \mathrm{kDa}$ ). Because no information about $\mathrm{pI}$ is gained from a one-dimensional SDS-PAGE gel, it was assumed that the intensely stained band resulted from an increase in transcription elongation factor. Perhaps, an excess of Mn may have positive effects on transcription especially with enzymes utilizing $\mathrm{Mn}$ as a cofactor.

Protein profiling by 2D gel electrophoresis. The vast majority of borrelial proteins are basic proteins with an isoelectric point above 7.5; therefore, protein analysis by 
conventional 2D gel electrophoresis was hampered by cathodic drift resulting in poor and inaccurate protein separation. To circumvent these difficulties, most borrelial protein research involving 2D gels has been accomplished using NEPHGE (Norris et al., 1992; Marconi et al., 1993a; Carroll and Gherardini, 1995; Carroll et al., 1999; Carroll et al., 2000). In the first dimension of NEPHGE, proteins are separated by pI, but the electrophoresis does not reach equilibrium (i.e., the proteins do not necessarily reach their pI, but are within a close range). Incomplete separation in this dimension results in horizontal streaks across the gel. Electrophoresis in the second dimension results in separation by molecular weight. To investigate differences in protein expression of cells cultured in an Ferestricted medium and Mn-enriched medium, Sh-2-82 stationary phase cells from these cultures were analyzed by NEPHGE. One hundred micrograms of protein was loaded onto tube gels with a pI gradient from 6-11 and electrophoresed in the first dimension, followed by SDS-PAGE and silver staining (Figure 21). No considerable differences were observed upon visual inspection of gels from different culture conditions. Ten proteins in Figure 21A were identified by MALDI-TOF (Figure 21, Table 5). Gel images were scanned and analyzed using Z3 software (Compugen), which calculates density values based on number of pixels within a given area. Two or more gel images were overlaid to produce a composite image. Densitometric analysis on the first gel image was performed and proteins were visualized as green spots. Similarly, the second gel image was visualized as pink spots. The software integrated the colored areas and the matching spots appeared as gray to black depending on density. A range of 80 to 150 protein spots was detected on each of the 2D gels. Several proteins were identified as being differentially expressed in Sh-2-82 cells 
Figure 21. Protein profiles of Sh-2-82 under different metal conditions using NEPHGE. B. burgdorferi Sh-2-82 cells were cultured in Fe-restricted (BSK-E plus $10 \mathrm{mM}$ dipyridyl) and Mn-enriched (BSK-E plus $100 \mu \mathrm{M} \mathrm{MnCl}_{2}$ ) media. Approximately $100 \mu \mathrm{g}$ CFE protein from stationary-phase cultures were loaded onto the tube gel (pH 6 to 11). Proteins were separated by NEPHGE in the first dimension and by 10\% SDS-PAGE in the second dimension. The gels were silver stained. A. BSK-E; B. BSK-E plus $10 \mathrm{mM}$ dipyridyl (Ferestrictive medium); C. BSK-E plus $100 \mu \mathrm{M} \mathrm{MnCl}$ ( $\mathrm{Mn}$ - enriched medium). 
A. BSK-E (Control)

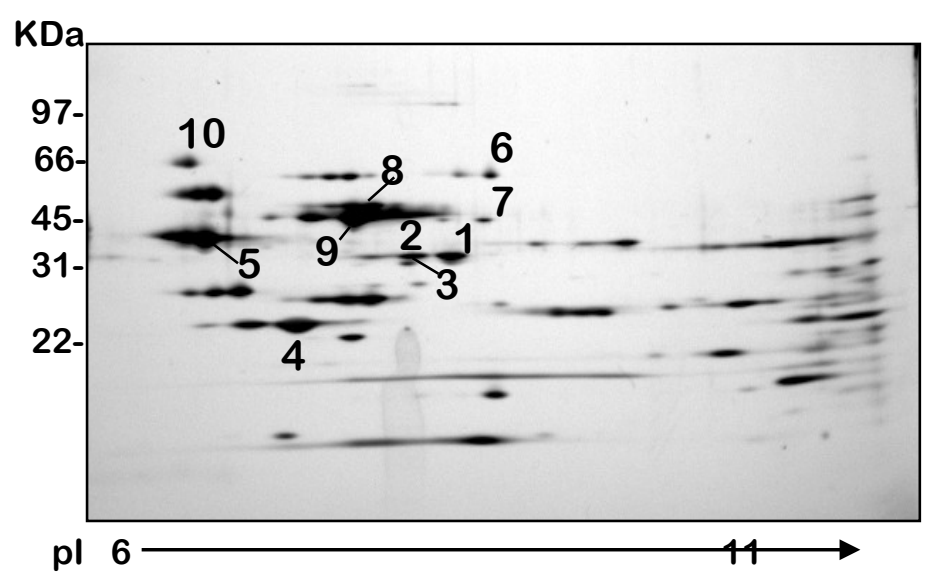

B. BSK-E + 10 mM dipyridyl

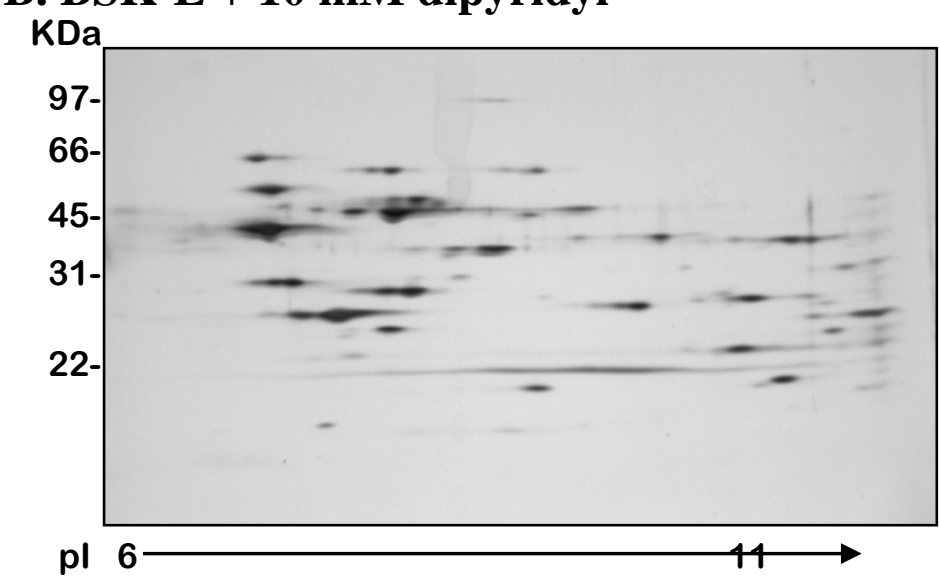

C. BSK-E $+100 u M \mathrm{MnCl}_{2}$

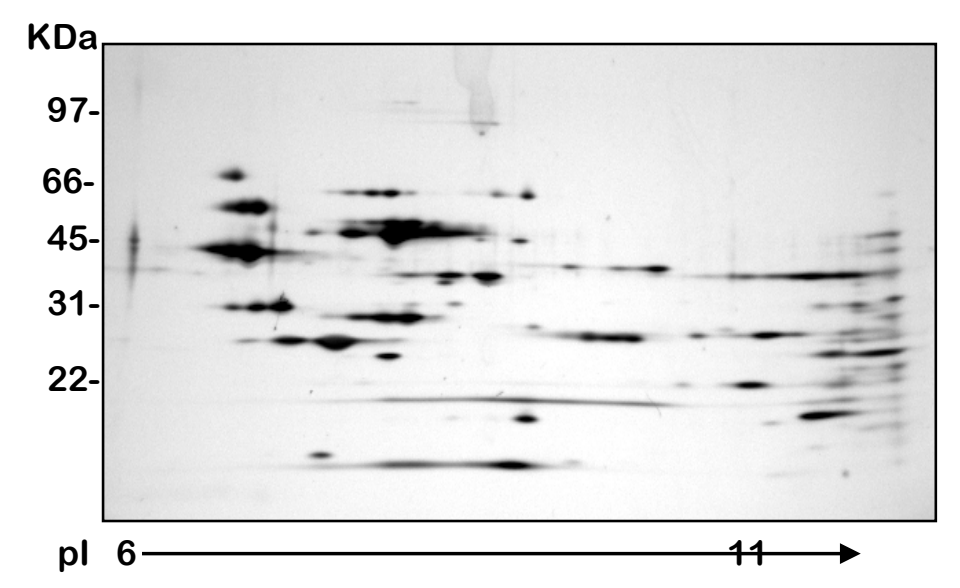


Table 5. Protein identification from 2D-gel electrophoresis

\begin{tabular}{lllll}
\hline Spot & Protein & Locus $^{\mathrm{a}}$ & MW $^{\mathrm{b}}$ & $\mathrm{pI}^{\mathrm{c}}$ \\
\hline 1 & Outer surface protein B & BBA16 & 31,774 & 8.82 \\
2 & Outer surface protein A & BBA15 & 29,455 & 6.72 \\
3 & Outer surface protein A & BBA15 & 29,455 & 6.72 \\
4 & Outer surface protein C & BBB19 & 22,330 & 7.03 \\
5 & Flagellin & BB147 & 35,765 & 5.51 \\
6 & Periplasmic serine protease & BB104 & 53,084 & 9.89 \\
7 & Glyceraldehyde phosphate & BB057 & 36,254 & 8.15 \\
& dehydrogenase & & & \\
8 & Phosphoglycerate kinase & BB056 & 42,345 & 6.80 \\
9 & L-lactate dehydrogenase & BB087 & 34,770 & 6.89 \\
10 & Heat shock protein & BB649 & 83,692 & 5.68 \\
\hline
\end{tabular}

${ }^{a}$ Gene locus from TIGR genome database

${ }^{\mathrm{b}}$ Estimated molecular weight in Daltons from TIGR sequence data

${ }^{\mathrm{c}}$ Estimated isoelectric point from TIGR sequence data 
cultured in Fe-restricted or Mn-enriched media as compared to the BSK-E control (Figure 21). Computer-aided detection of differentially expressed proteins is shown in Figure 22. Because NEPHGE does not allow equilibrium between pI of the protein and $\mathrm{pH}$ of the gel gradient, the computer analysis was less effective in identifying individual spots due to horizontal streaking.

Dramatic improvements in 2D gel electrophoresis have been recognized with the advent of immobilized pH gradient (IPG) gels for the first dimension. These gels are cast as thin gels on a plastic support and are not subject to electrophoretic distortion (cathodic drift) or physical distortion during handling as with tube gels (Westermeier, 1997). Using IPG gels with a pH gradient of 3-10, stationary phase cells from Fe-restricted and Mn-enriched cultures were evaluated by IEF 2D gel analysis and silver staining. Better resolution of individual protein spots was observed with these gels; however, the gels appeared to contain less protein than previously observed with NEPHGE. As stated earlier, most borrelial proteins would be positioned in the basic region of the gel; therefore, a narrow basic $\mathrm{pH}$ range would have been more informative than a wide range with a substantial acidic region such as the IPG gel 6-11 used in the NEPHGE experiment. Composite gels from each condition, Fe-restriction and Mn-enrichment, were overlaid with a composite gel obtained from extracted proteins from Sh-2-82 cells cultured in BSK-E, as a control (Figure 24). Very few differences were observed upon visual inspection of the gels. To correlate the proteins identified by MALDI-TOF from the NEPHGE gels, stained spots were excised from the gel and processed for peptide profiling (Figures 21 and 23, Table 5). 
Figure 22. Differential protein expression of Sh-2-82 in response to iron-restriction and manganese-enrichment. Silver-stained gels were scanned and gel images were analyzed using Z3 software. This software overlays two or more gel images to produce a composite image. Matching protein spots are seen as gray to black areas depending on density.

Proteins identified only in the first (control) gel image are visualized as green spots; whereas, proteins only in the second (test condition) gel image appear pink. A. The protein profile of cells cultured in BSK-E was compared with cells cultured in BSK-E plus $10 \mathrm{mM}$ dipyridyl. B. The protein profile of cells cultured in BSK-E was compared with that of cells cultured in BSK-E plus $100 \mu \mathrm{M} \mathrm{MnCl}_{2}$. Blue arrows indicate areas of differential expressed proteins. 
A. BSK-E vs. BSK-E plus $10 \mathrm{mM}$ dipyridyl (Fe-restricted)

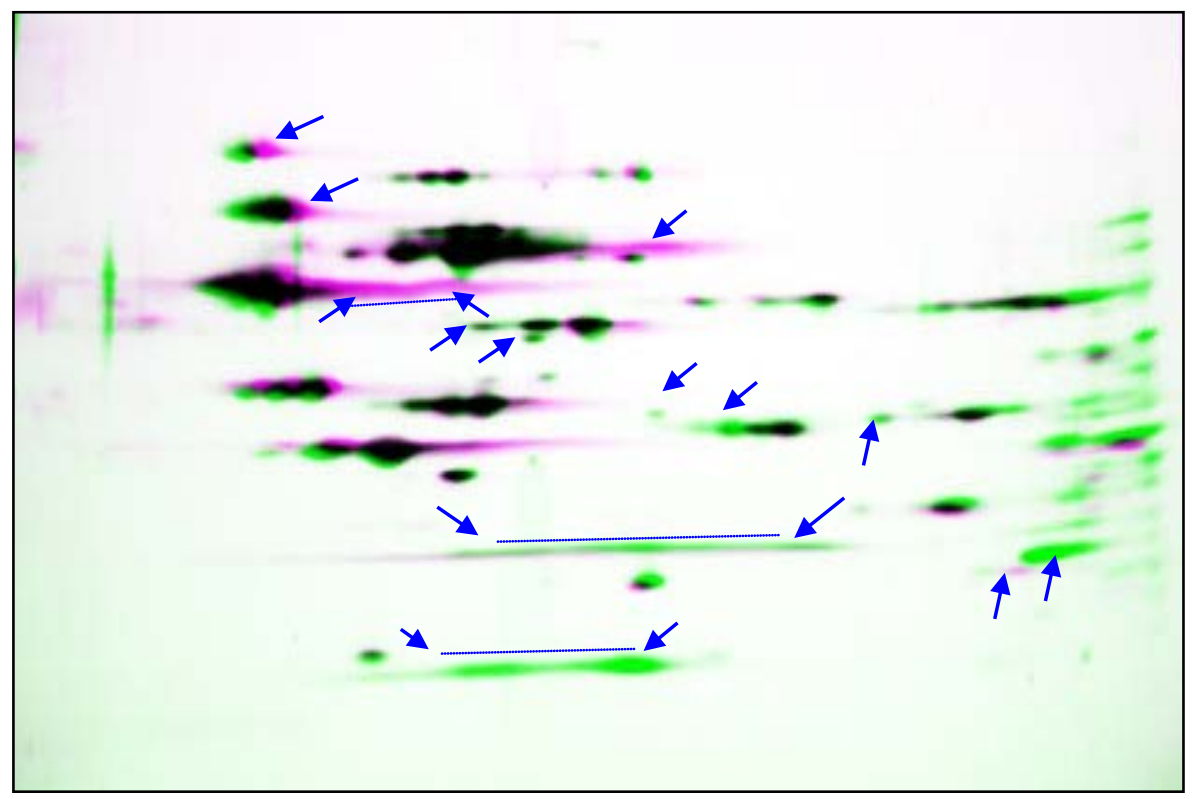

B. BSK-E vs. BSK-E plus $100 \mu \mathrm{M} \mathrm{MnCl}_{2}$ (Mn-enriched)

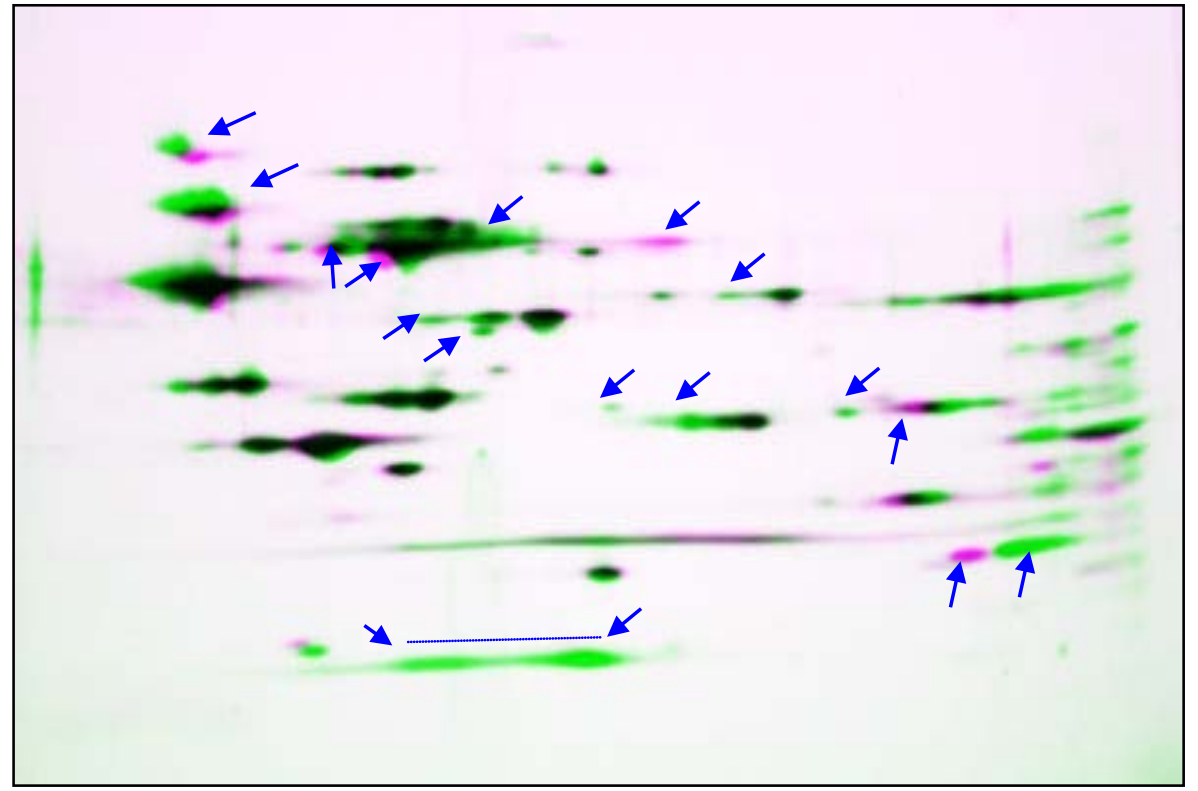


Figure 23. Protein profiles of Sh-2-82 under different metal conditions using immobilized pH gradient gels and SDS-PAGE. B. burgdorferi Sh-2-82 cells were cultured in Fe-restricted (BSK-E plus $10 \mathrm{mM}$ dipyridyl) and Mn-enriched (BSK-E plus $100 \mu \mathrm{M}$ $\mathrm{MnCl}_{2}$ ) media. Approximately $100 \mu \mathrm{g}$ stationary phase borrelial protein was loaded onto the IPG strip (pH 3 to 10). Proteins were separated by IEF in the first dimension and by $10 \%$ SDS-PAGE in the second dimension. The gels were silver stained. A. BSK-E (Control); B. BSK-E plus dipyridyl (Fe-restrictive); C. BSK-E plus $100 \mu \mathrm{M} \mathrm{MnCl}_{2}$ (Mn-enriched). 


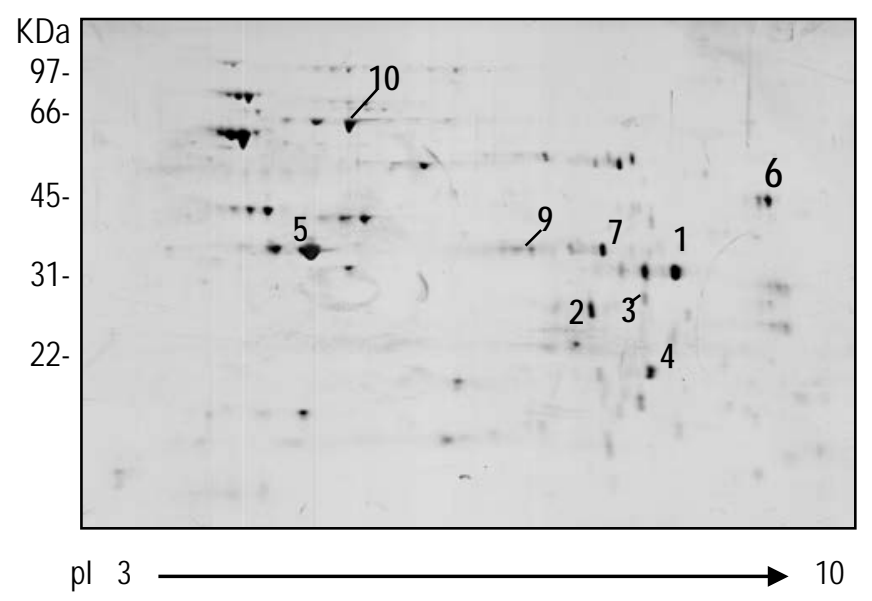

B. BSK-E plus $10 \mathrm{mM}$ dipyridyl (Fe-restricted)

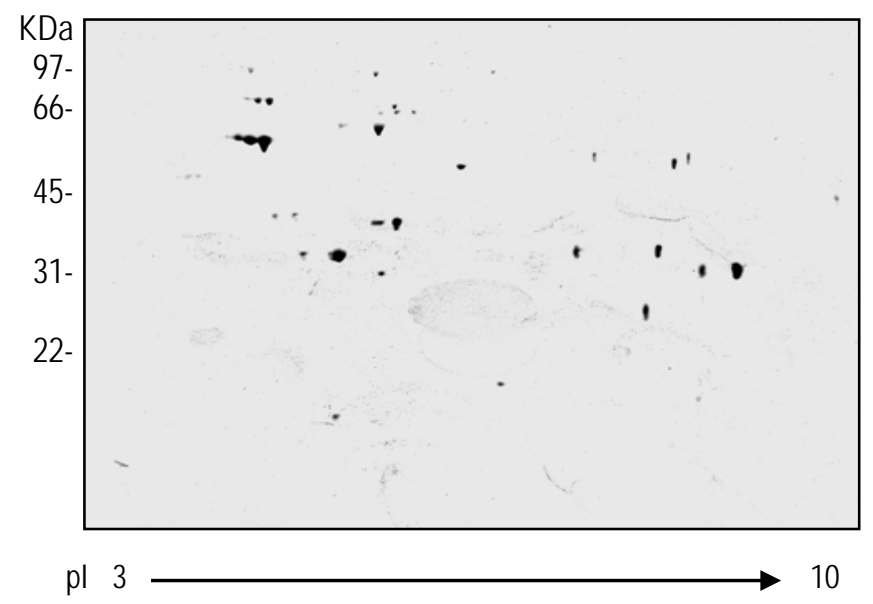

C. KSKK-E plus 100 \#M MnCl $($ Mn-enriched)

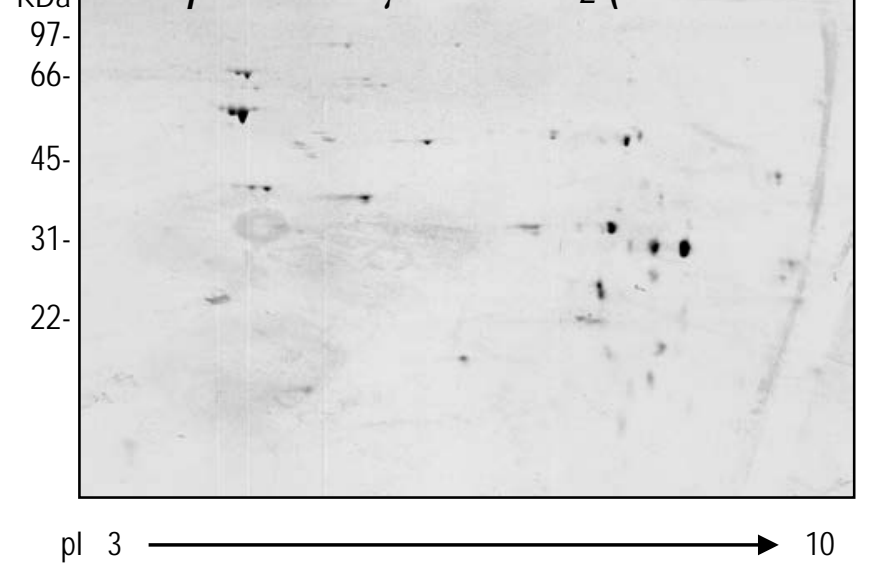


Figure 24. Differential protein expression of Sh-2-82 in response to iron-restriction and manganese-enrichment using IEF and SDS-PAGE. Silver stained gels were scanned and gel images were analyzed using Z3 software. This software overlays two or more gel images to produce a composite image. Matching protein spots are seen as gray to black areas depending on density. Proteins identified only in the first (control) gel image are visualized as green spots; whereas, proteins only in the second (test condition) gel image appear pink. A. The protein profile of cells cultured in BSK-E was compared with that of cells cultured in BSK-E plus $10 \mathrm{mM}$ dipyridyl. B. The protein profile of cells cultured in BSK-E was compared with that of cells cultured in BSK-E plus $100 \mu \mathrm{M} \mathrm{MnCl}_{2}$. Blue arrows indicate areas of differentially expressed proteins. 
A. BSK-E vs. BSK-E plus $10 \mathrm{mM}$ dipyridyl (Fe-restricted)

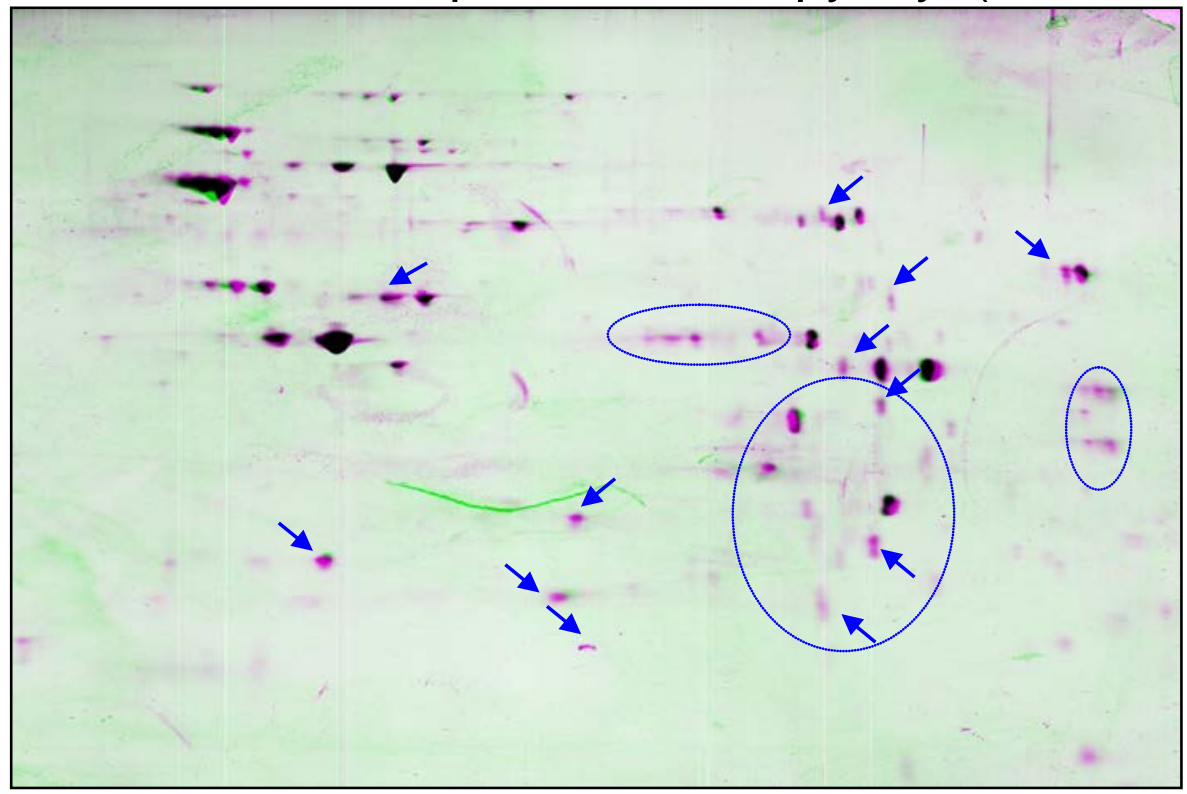

B. BSK-E vs. BSK-E plus $100 \mu \mathrm{M} \mathrm{MnCl} 2$ (Mn-enriched)

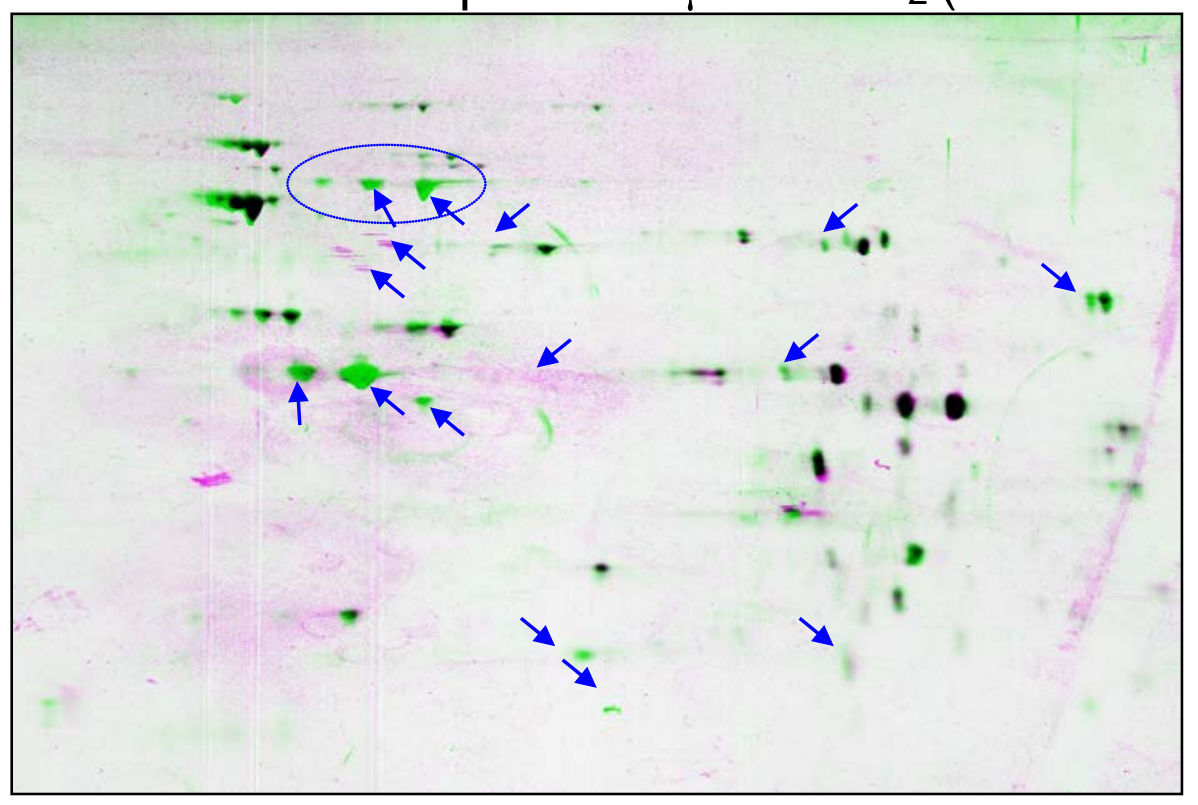


The proteins that were identified by peptide sequencing were primarily associated with the membrane. The prominent outer membrane proteins, OmpA, OmpB, and OmpC, were readily detectable on the gel and their location was confirmed by peptide sequencing of the spots. Two periplasmic proteins, serine protease and flagellin, were identified, along with three metabolic enzymes and the heat shock protein, GroEL. The predicted isoelectric points of the identified proteins ranged from 5.51 to 9.89, while the molecular weights ranged from 29.5 to $83.6 \mathrm{kDa}$. Although all the proteins identified on the gels were not sequenced and identified, the proteins of interest to this project, SecA and SOD were probably not detectable with these gel preparations. Because high molecular weight proteins are difficult to separate using 2-D gel techniques, the B. burgdorferi SecA at $102 \mathrm{kDa}$ was not visible on the gel. On the other hand, the relatively low MW (24 kDa) of B. burgdorferi SOD is detectable on 2-D gels. However, a shortcoming of 2-D gel electrophoresis is that only the most prominently expressed proteins are usually visualized with total protein extracts. The SOD activity studies indicate that the B. burgdorferi SOD is not highly expressed; thereby, the presence of SOD would not be visible using silver staining. Because the majority of $B$. burgdorferi proteins are membrane proteins, cell fractionation resulting in an enriched cytoplasmic protein extract would enhance the likelihood of identifying low expressed proteins such as SOD. Also, with an enriched cytoplasmic fraction, a very narrow pI range in the first dimension could be used to seek out many proteins that are obscured by the abundant basic proteins. The predicted isoelectric points of SecA and SOD are 7.9 and 6.7, respectively. Concentrated cytoplasmic protein extracts of B. burgdorferi would greatly enhance the likelihood of identifying SecA and SOD on protein gels. 


\section{DISCUSSION}

Although B. burgdorferi is a microaerophilic organism and has limited exposure to environmental $\mathrm{O}_{2}$, the bacterium must still defend against ROIs generated during cellular metabolism. Whitehouse et al. (1997) identified SOD as the sole oxidative defense mechanism in B. burgdorferi. The absence of catalase and peroxidase activities (Whitehouse et al., 1997) was confirmed by the lack of catalase and peroxidase genes in the sequenced genome (Fraser et al., 1997). Therefore, SOD expression and activity were thought to be essential under normal culture conditions as well as stress conditions. The present study sought to investigate the expression and regulation of the B. burgdorferi sod gene in the virulent strain, Sh-2-82.

Transcription of bacterial sod genes is tightly regulated generally from a $\sigma^{70}$-like promoter as a monocistronic message. The quest to identify such regulation in the $B$. burgdorferi Sh-2-82 sod began with the available genomic sequence data (Fraser et al., 1997). The sod ORF began only 17 bases downstream from the stop codon of the preceding gene, $\sec A$, suggesting that the sodA promoter sequence would be located within the carboxy terminus of secA. Initial experiments using Northern blotting with gene-specific probes to determine the size of the sod transcript did not yield the expected band at $0.6 \mathrm{~kb}$. Rather, a band was observed at $3.7 \mathrm{~kb}$, which was of sufficient size to encode both the 609-bp sod ORF plus the 2,697-bp ORF of secA (Figure 1). This was the first indication that $\sec A$ and sod were cotranscribed, which was later confirmed by RT-PCR (Figure 2). Probing of the amino termini and upstream regions of secA and sod using PE analyses (Figure 4 and Figure 6) indicated that a $\sigma^{70}$-like promoter upstream of $\sec A$ was responsible for transcription of this bicistronic operon. This organization is unusual because normally sod is under strict 
transcriptional regulation by its own promoter and cis-acting elements, while $\sec A$ is regulated at the translational level (Salavati and Oliver, 1995). In E. coli, SecA binds to its own mRNA transcript, which inhibits ribosomes from binding and stalls translation (Salvati and Oliver, 1995). If B. burgdorferi secA were regulated in this same manner, sod would be transcribed and translated with minimal regulation. The RBS upstream of the sod ORF would remain available although the secA transcript was not translated. Potentially, the sod transcript may exhibit a longer half-life because of the stabilizing effects from the binding of secA. An increased half-life might account for the sod transcript levels being consistently higher than $\sec A$ as measured by RPA (Figure 16). However, the secA probe corresponded to nucleotides located in the middle of the ORF, which should not have been affected by the binding at the amino terminus unless complex secondary structures were formed.

Additional studies investigated possible transcriptional regulation by metals of the $B$. burgdorferi secA-sod operon. To rule-out growth phase differences in the transcription of these two genes, relative abundance of $\sec A$ and sod transcripts were quantified using RPA and densitometric analysis software (Figure 16). The highest mRNA expression was observed in stationary phase cells; therefore, metalloregulation of secA-sod message was investigated in B. burgdorferi cells grown to stationary phase. Obviously, Fe was the first candidate to be tested because of the well-documented Fur regulation of sod genes (Griggs and Konisky, 1989; Neiderhoffer et al., 1990; Stojiljkovic et al., 1994; Zheng et al., 1999). In addition, a fur homolog had been identified in the B. burgdorferi genome (Fraser et al., 1997), as well as an earlier study had identified a membrane protein that bound transferrin (Carroll et al., 1996). The Fur family includes metal-ion uptake regulator proteins that bind to the operator DNA and regulate transcription of metal-ion responsive genes. Fur-regulated 
promoters are defined by the presence of a consensus sequence known as the Fur box (Escolar et al., 1999). Potential Fur boxes were identified upstream of both secA and sod ORFs (Figure 8). However, Escolar et al. (1999) observed that a minimum of three repeats of the hexamer, $\mathrm{N}-\mathrm{AT}^{\mathrm{A}} / \mathrm{TAT}$, regardless of orientation, was required for effective Fur binding. Additional studies using gel retardation assays and DNA footprinting would provide valuable information on potential Fur-regulated promoters (Frechon and Cam, 1994).

The mechanistic details of Fur-regulation are puzzling because Fur binds several divalent cations other than $\mathrm{Fe}^{+2}$ in vitro. Studies have suggested that Fur preferentially binds Fe, but also binds $\mathrm{Mn}\left(\mathrm{Mn}^{2+}\right)$, cobalt $\left(\mathrm{Co}^{2+}\right)$, copper $\left(\mathrm{Cu}^{2+}\right)$, cadmium $\left(\mathrm{Cd}^{2+}\right)$, and $\mathrm{Zn}\left(\mathrm{Zn}^{2+}\right)$ (Adriat et al, 1999; Bagg and Neilands, 1987; deLorenzo et al., 1987; Gonzalez et al., 1999, Hahn et al., 2000; vanVliet et al., 1999). Recently, Althaus et al. (1999) identified E. coli Fur as a Zn metalloprotein containing either a single Zn ion ( $\mathrm{Zn}_{1}$ Fur) or two (Zn2Fur) with similar DNA binding activity. The tightly associated $\mathrm{Zn}$ ion is coordinated with two sulfur ligands, Cys92 and Cys95, and two nitrogen or oxygen ligands (Jacquamet et al., 1998). The two cysteine residues are known to be essential for DNA-binding activity (Coy et al., 1994) and are conserved in most Fur-homologous proteins (Hahn et al., 2000). However, only genomic sequence data are available on the fur homolog in B. burgdorferi (Fraser et al., 1997). The borrelial Fur may function with Zn bound rather than Fe.

The borrelial Fur may belong also to another group of Fur-related proteins, such as Zur (Zn uptake regulator) or PerR (peroxide regulator). In E. coli, Zur is encoded by yibK and has only 27\% sequence identity with Fur (Blattner et al., 1993). The high-affinity Znuptake system znuABC is regulated by the repressor, Zur. This system was one of the first examples showing that the Fur protein family is not specific for $\mathrm{Fe}^{2+}$ as corepressor. In 
addition, a Fur-like protein with Zn as a corepressor of high- and low-affinity Zn uptake systems has been found in B. subtilis (Gaballa and Helmann, 1998). Likewise, genes with gene products significantly similar to Zur have been identified in Vibrio (Zheleznova et al., 2000) and in Pseudomonas (Rossbach et al., 2000).

In gram-positive bacteria, another Fur-related protein has emerged as a regulator of peroxide stress (Chen et al., 1995; Bsat et al., 1998, vanVliet et al., 1999; Horsburgh et al., 2001). Studies in $B$. subtilis with $\mathrm{H}_{2} \mathrm{O}_{2}$ treatment and metal ion limitation showed an increased expression of catalase, alkyl hydroperoxidase, and a Mn-response protein (Dowds, 1994). Analysis of the promoters of these genes identified a consensus sequence similar to a Fur box; this sequence was termed a Per box. Bsat et al. (1998) found that the gene product of $y d k L$ demonstrated Fe uptake-repressive activities as well as structural similarities to Fur. The $y g a G$ gene product encoded the PerR protein, which acts as a metal-binding repressor and binds to peroxide stress-related promoters. As with Fur, PerR requires a divalent metal ion to activate its DNA-binding activity and several studies have shown that both $\mathrm{Mn}$ and Fe can fulfill this metal requirement (Chen et al., 1993; Chen et al., 1995; Bsat et al., 1996). Similar to B. subtilis, S. aureus encodes three Fur homologs, Fur, PerR, and Zur (Horsburgh et al., 2001; Lindsay and Foster, 2001). PerR functions as a peroxide stress regulator that controls Fe uptake and storage proteins by interacting with Fur (Horsburgh et al., 2001). In light of these recent studies on Fur-related proteins, it would be of value to analyze the borrelial Fur more closely for similarities to PerR and Zur.

Recent characterization of a Mn-dependent regulatory protein, TroR, in T. pallidum suggested that Mn was responsible for metal regulation in this spirochete (Posey et al., 1999). In assays to determine the metal specificity of this regulatory protein, only $\mathrm{Mn}^{+2}$ was 
able to activate TroR. This observation is in marked contrast to the activation profiles for other metal-dependent regulatory proteins such as Fur or DtxR, which are activated by various divalent heavy metals such as $\mathrm{Fe}^{2+}, \mathrm{Zn}^{2+}, \mathrm{Cu}^{2+}, \mathrm{Ni}^{2+}$, or $\mathrm{Co}^{2+}$. Two of the five $\mathrm{Fe}-$ binding residues of DtxR were different in the TroR sequence, which would possibly explain the difference in metal specificity. TroR is the fifth gene in a transport-related operon (Fraser et al., 1998). The first four genes of this operon encode a putative ABC metal transport system (troA-D). TroA has been characterized as a periplasmic Zn-binding protein (Lee et al.,1999). Although such a system has not yet been identified in B. burgdorferi, it is possible that one of the 32 putative ABC transport proteins identified in the genome (Fraser et al., 1997) may function similarly to T. pallidum TroA with analogous regulation from a Mnregulatory protein.

The genomes of $T$. pallidum and B. burgdorferi are similar in that they contain very few genes that encode metalloproteins. Analysis of the complete genome sequence of $B$. burgdorferi identified sodA, fur, and napA (putative neutrophil-activating protein). These proteins require divalent cations as cofactors such as $\mathrm{Fe}^{2+}$ or $\mathrm{Mn}^{2+}$. Posey and Gherardini (2000) determined the intracellular Fe concentration in B. burgdorferi to be less than 10 atoms per cell as measured by inductively coupled plasma-emission mass spectroscopy. No growth restriction was observed when $B$. burgdorferi were grown in the presence of Fe chelators (Posey and Gherardini, 2000). In this study, Desferal and dipyridyl did not restrict growth in BSK-II medium (Figure 9). To avoid possible Fe contamination with BSA and rabbit serum, BSK-E medium, which includes the additional lipids from non-blood sources, was used. The BSK-E medium was treated also with Chelex-100 to remove all divalent cations and then the BSK-E-Clx medium was supplemented with specified amounts of 
$\mathrm{MgCl}_{2}$. $\mathrm{MnCl}_{2}, \mathrm{ZnCl}_{2}$. and $\mathrm{FeCl}_{2}$. As observed previously (Posey and Gherardini, 2000), $B$. burgdorferi requires a combination of Mg, Mn, and Zn cations for growth in BSK-E-Clx. The addition of Fe did not impart any growth advantages alone or in combination with the above-mentioned cations. Taken together with the genomic data, these studies suggest a lack of a role for Fe in B. burgdorferi.

If Fe concentrations were below physiologically relevant concentrations as demonstrated by Posey and Gherardini (2000), this finding would suggest that the metal enzyme cofactor in B. burgdorferi SOD would be Mn. The genomic sequence more closely matches to a MnSOD (Fraser et al., 1997), but the physiological assays indicate an FeSOD (Whitehouse et al., 1997). The possibility exists that the borrelial SOD is cambialistic, meaning that the protein may function with either Fe or Mn, whichever is supplied in the medium. To test this possibility, the cultures were cultured in Fe-restricted, Mn-restricted, and Mn-enriched media. Iron-restriction did not affect culture growth (Figure 9) nor transcription of secA-sod operon (Figure 18), but no SOD activity was detected by gel electrophoresis (Figure 19) or spectrophotometrically. On the other hand, Mn-restriction significantly lowered growth (Figure 14, Figure 17), and overall transcription was reduced (Figure 18). Although Mg and Zn were added back to BSK-E-Clx medium, total restoration of necessary divalent cations may not have occurred, thereby, reducing transcription efficiency. No SOD activity was observed in low levels of Mn; however, at $100 \mu \mathrm{M} \mathrm{MnCl}_{2}$, SOD activity was observed (Figure 19). It should be noted that $400 \mu \mathrm{g}$ of borrelial CFE were needed to demonstrate SOD activity in gels as compared to $50 \mu \mathrm{g}$ of $E$. coli CFE. This extremely low level of activity may be explained by the lack of a strong promoter immediately upstream of the borrelial sod ORF. 
Protein profiling by 2-D gel electrophoresis coupled with protein identification by mass spectrophotometry of borrelial proteins has the potential to correlate the known genomic sequence to translated and processed proteins that are synthesized by borreliae. This study sought to identify the B. burgdorferi SecA and SOD proteins by 2-D gel electrophoresis. Proteins were extracted from cells in early stationary phase, the time at which both proteins should be expressed. The genes are highly expressed during this time as measured by RPA. However, SecA and SOD are not highly expressed relative to the abundant outer membrane proteins, which probably obscured the identification of many lower-expressed proteins. Secondly, the study compared the protein profiles from cells grown in Fe-restricted medium and Mn-enriched medium with cells cultured in normal metal-competent medium. Although very few protein differences were observed from the different culture conditions, fewer basic proteins were visualized on NEPHGE gels representative of cells deprived of Fe. Separation using IEF in the first dimension decreased horizontal streaking of proteins and resulted in sharper resolution of protein spots; however, fewer proteins appeared on those gels. Changes in metal content seem to have an effect on the presence of periplasmic proteins (flagellin and serine protease, Figures 23, 24) in the CFEs separated by IEF followed by PAGE; however, these changes were not apparent by NEPHGE separation (Figures 21, 22). To clarify changes in protein profiles, cell fractionation would be needed to concentrate and enrich samples to assess relative abundance of proteins within each fraction. By doing so, the major membrane proteins would be removed from the cytoplasmic and/or periplasmic protein extracts allowing for minor protein changes to be analyzed. 
A 2-D gel database for pathogenic microorganisms has recently been constructed and available via the internet (http://mpiib-berlin.mpg.de/2D-PAGE). Borrelial 2-D gels have been submitted to this database for inclusion along with the identity of $20 \mathrm{~B}$. garinii antigens (Jungblat et al., 1999). As more proteins are identified and the normal protein pattern for Borrelia is established, this technology may become more useful in detection of immunogenic antigens expressed in response to different environmental conditions within the host and the microorganism.

Future studies involving the gene products of the secA-sod operon might focus on the localization of these proteins and their role in virulence. Very little data are available on the general secretory pathway in spirochetes. In other bacteria, SecA plays an integral role in passage of proteins to the outer membrane. It is believed that B. burgdorferi uses SecAdependent secretion for its multitude of outer membrane lipoproteins (Haake, 2000). The spirochete may vary its antigenic composition of surface lipoproteins by sensing and adapting to tick and mammalian environments. The spirochete in the unfed tick expresses OspA, but not OspC. When the tick takes a blood meal, OspC is expressed and OspA is not. In contrast, in the mammalian host, OspC is expressed early and OspA is expressed late. Additional outer surface proteins encoded on plasmids may fluctuate in expression depending on the presence of certain plasmids. The loss of plasmids upon culture passage has been associated with a loss of infectivity and virulence, but a specific plasmid or gene required for virulence has not been identified. The elucidation of how SecA functions in the secretion process of immunogenic proteins such as Osps would yield important information on antigenic variability observed among infectious isolates. Potential studies that would 
inhibit SecA would be of extreme value in answering questions on pathogenesis associated with spirochetal membrane topology. 


\section{LITERATURE CITED}

Adriat, A., I. Jacquamet, L. LePape, A. Gonzalez de Peredo, D. Aberdam, J. L. Hazemann, J. M. Latour, and I. Michaud-Soret. 1999. Spectroscopic and saturation magnetization properties of the manganese-and cobalt-substituted Fur (Ferric uptake regulation) protein from Escherichia coli. Biochemistry 38:6248-6260.

Afzelius, A. 1910. Verhandlungen der dermatologischen Gessellschaft zu Stockholm. Arch. Dermatol. Syphil. 101:404.

Akita, M., S. Sasaki, S. Matsuyama, and S. Mizushima. 1990. SecA interacts with secretory proteins by recognizing the positive charge at the amino terminus of the signal peptide of Escherichia coli. J. Biol. Chem. 265:8164-8169.

Aldsworth, T. G., E. R. Dodd, and G. S. Stewart. 1999. Induction of rpoS in Salmonella typhimurium by nutrient-poor and depleted media is slower than that achieved by a competitive microflora. Lett. Appl. Microbiol. 28:255-257.

Althaus, E. W., C. E. Outten, K. E. Olson, and T. V. O'Halloran. 1999. The ferric uptake regulation (Fur) repressor is a zinc metalloprotein. Biochemistry 38:6559-6569.

Ananthaswamy, H. N., and A. Eisenstark. 1977. Repair of hydrogen peroxide-induced single-strand breaks in Escherichia coli deoxyribonucleic acid. J. Bacteriol. 130:187191.

Anderson, J. F., L. A. Magnareli, R. B. LeFebvre, T. G. Andreadis, J. B. McAninch, G. C. Perng, and R. C. Johnson. 1989. Antigenically variable Borrelia burgdorferi isolated from cottontail rabbits and Ixodes dentatus in rural and urban areas. J. Clin. Microbiol. 27:13-20.

Asbrink, E., and A. Hovmark. 1987. Cutaneous manifestations in Ixodes-borne Borrelia spirochetosis. Int. J. Dermatol. 26:215-223.

Austin, F. E., J. T. Barbieri, R. E. Corbin, K. E. Grigas, and C. D. Cox. 1981. Distribution of superoxide dismutase, catalase, and peroxidase activities among Treponema pallidum and other spirochetes. Infect Immun. 33:372-379. 
Austin, F. E. 1993. Maintenance of infective Borrelia burgdorferi Sh-2-82 in 4\% oxygen5\% carbon dioxide in vitro. Can. J. Microbiol. 39:1103-1110.

Bagg, A., and J. B. Neilands. 1987. Ferric uptake regulation protein acts as a repressor, employing iron (II) as a cofactor to bind the operator of an iron transport operon in Escherichia coli. Biochemistry 26:5471-5477.

Barbour, A. G. 1984. Isolation and cultivation of Lyme disease spirochetes. Yale J. Biol. Med. 57:521-525.

Barbour, A. G. 1988. Plasmid analysis of Borrelia burgdorferi, the Lyme disease agent. J. Clin. Microbiol. 26:474-478.

Barbour, A. G. 1996. Does Lyme disease occur in the south?: a survey of emerging tickborne infections in the region. Am. J. Med. Sci. 311:34-40.

Barbour, A. G. 1998. Fall and rise of Lyme disease and other Ixodes tick-borne infections in North America and Europe. Brit. Med. Bull. 54:647-658.

Barbour, A. G., and C. F. Garon. 1987. Linear plasmids of the bacterium Borrelia burgdorferi have covalently closed ends. Science 237:409-411.

Barbour, A. G., and S. F. Hayes. 1986. Biology of Borrelia species. Microbiol. Rev. 50:381-400.

Barbour, A. G., G. O. Maupin, G. J. Teltow, C. J. Carter, and J. Piesman. 1996. Identification of an uncultivable Borrelia species in the hard tick Amblyomma americanum: possible agent of a Lyme disease-like illness. J. Infect. Dis. 173:403409.

Barbour, A. G., S. L. Tessier, and S. F. Hayes. 1984. Variation in a major surface protein of Lyme disease spirochetes. Infect. Immun. 45:94-100.

Baril, C., C. Richaud, G. Baranton, and I. S. Girons. 1989. Linear chromosome of Borrelia burgdorferi. Res. Microbiol. 140:507-516. 
Barthold, S. W., and L. K. Bockenstedt. 1993. Passive immunizing activity of sera from mice infected with Borrelia burgdorferi. Infect. Immun. 61:4696-4701.

Barthold, S. W., M. S. deSouza, J. L. Janotka, A. L. Smith, and D. H. Persing. 1993. Chronic Lyme borreliosis in the laboratory mouse. Am. J. Pathol. 143:959-972.

Beall, B., and J. Lutkenhaus. 1987. Sequence analysis, transcriptional organization, and insertional mutagenesis of the envA gene in Escherichia coli. J. Bacteriol. 169:54085415.

Beauchamp, C., and I. Fridovich. 1971. Superoxide dismutase: improved assays and an assay applicable to acrylamide gels. Anal. Biochem. 44:276-287.

Belman, A. L., M. Iyer, P. K. Coyle, and R. Dattwyler. 1993. Neurologic manifestations in children with North American Lyme disease. Neurology 43:2609-2614.

Benach, J. L., H. B. Fleit, G. S. Habicht, J. L. Coleman, E. M. Bosler, and B. P. Lane. 1984. Interactions of phagocytes with the Lyme disease spirochete: role of the Fc receptor. J. Infect. Dis. 150:497-507.

Benov, L. T., and I. Fridovich. 1994. Escherichia coli expresses a copper- and zinccontaining superoxide dismutase. J. Biol. Chem. 269:25310-25314.

Beyer, W. F., and I. Fridovich. 1987. Effect of hydrogen peroxide on the iron-containing superoxide dismutase of Escherichia coli. Biochemistry 26:1251-1257.

Black, M. T. 1993. Evidence that the catalytic activity of prokaryote leader peptidase depends upon the operation of a serine-lysine catalytic dyad. J. Bacteriol. 175:49574961.

Black, M. T. , J. G. Munn, and A. E. Allsop. 1992. On the catalytic mechanism of prokaryotic leader peptidase 1. Biochem. J. 282:539-543.

Blattner, F. R., V. Burland, G. Plunkett III, H. J. Sofia, and L. Daniels. 1993. Analysis of the Escherichia coli genome. IV. DNA sequence of the region from 89.2 and 92.8 minutes. Nucleic Acids Res. 21:5408-5417. 
Blattner, F. R., G. Plunkett, C. A. Bloch, N. T. Perna, V. Burland, M. Gregor, N. W. Davis, H. A. Kirkpatrick, M. A. Goeden, D. J. Rose, B. Mau, and Y. Shao. 1997. The complete genome sequence of Escherichia coli K-12. Science 277:1453-1473.

Bledsoe, H. A., J. A. Carroll, T. R. Whelchel, M. A. Farmer, D. W. Dorward, and F. C. Gherardini. 1994. Isolation and partial characterization of Borrelia burgdorferi inner and outer membranes by using isopycnic centrifugation. J. Bacteriol. 176:7447-7455.

Borer, H. 1975. Spectrophotometric quantification of nucleic acids, p. 589 In G. D. Fasman (ed.), Handbook of Biochemistry and Molecular Biology. CRC Press, Cleveland, Ohio.

Bosler, E. M., J. L. Coleman, J. L. Benach, D. A. Massey, J. P. Hanrahan, W. Burgdorfer, and A. G. Barbour. 1983. Natural distribution of the Ixodes dammini spirochete. Science 220:321-322.

Brandt, M. E., B. S. Riley, J. D. Radolf, and M. V. Norgard. 1990. Immunogenic integral membrane proteins of Borrelia burgdorferi are lipoproteins. Infect. Immun. 58:983991.

Briat, J. F. 1992. Iron assimilation and storage in prokaryotes. J. Gen. Microbiol. 138:24752483.

Britton, L., and I. Fridovich. 1977. Intracellular localization of the superoxide dismutases of Escherichia coli: a reevaluation. J. Bacteriol. 131:815-820.

Broder, D. C., R. Lottenberg, G. O. vonMering, K. H. Johnston, and M. D. Boyle. 1991. Isolation of a prokaryotic plasmin receptor. Relationship to a plasminogen activator produced by the same microorganism. J. Biol. Chem. 266:4922-4928.

Brown, E. L., B. P. Guo, P. O'Neal, and M. Hook. 1999. Adherence of Borrelia burgdorferi. J. Biol. Chem. 274:26272-26278.

Bsat, N., and J. D. Helmann. 1999. Interaction of Bacillus subtilis Fur (Ferric uptake repressor) with the $d h b$ operator in vitro and in vivo. J. Bacteriol. 181:4299-4307. 
Bsat, N., A. Herbig, L. Casillas-Martinez, P. Setlow, and J. D. Helmann. 1998. Bacillus subtilis contains multiple Fur homologues: identification of the iron uptake (Fur) and peroxide regulon (PerR) repressors. Mol. Microbiol. 29:189-198.

Bult, C., O. White, G. Olsen, L. Zhou, R. D. Fleischmann, G. G. Sutton, J. A. Blake, L. M. Fitzgerald, R. A. Clayton, J. D. Gocayne, A. R. Kerlavage, B. A. Dougherty, J. Tomb, M. D. Adams, C. I. Reich, R. Overbeek, E. F. Kirkness, K. G. Weinstock, J. M. Merrick, A. Glodek, J. D. Scott, N. S. Geoghagen, J. F. Weidman, J. L. Fuhrmann, D. T. Nguyen, T. Utterback, J. M. Kelley, J. D. Peterson, P. W. Sadow, M. C. Hanna, M. D. Cotton, M. A. Hurst, K. M. Roberts, B. B. Kaine, M. Borodovsky, H. P. Klenk, C. M. Fraser, H. O. Smith, C. R. Woese, and J. C. Venter. 1996. Complete genome sequence of the methanogenic archaeon, Methanococcus jannaschii. Science 273:1058-1073.

Burgdorfer, W., A. G. Barbour, S. F. Hayes, O. Peter, and A. Aeschlimann. 1983. Erythema chronicum migrans - a tick-borne spirochetosis. Acta Tropica 40:79-83.

Burgdorfer, W. A., S. F. Hayes, J. L. Benach, E. Grunwalt, and J. P. Davis. 1982. Lyme disease - a tick-borne spirochetosis? Science 216:1317-1319.

Burgdorfer, W., S. F. Hayes, and D. Corwin. 1989. Pathophysiology of the Lyme disease spirochete, Borrelia burgdorferi, in Ixodid ticks. Rev. Infect. Dis. 6:S1442-S1450.

Burgess, R. R., A. A. Travers, J. J. Dunn, and E. K. F. Bautz. 1969. Factor stimulating transcription by RNA polymerase. Nature 221:43-44.

Burns, M. J. and M. B. Furie. 1998. Borrelia burgdorferi and interleukin-1 promote the transendothelial migration of monocytes in vitro by different mechanisms. Infect. Immun. 66:4875-4883.

Burns, M. J., T. J. Sellati, E. I. Teng, and M. B. Furie. 1997. Production of interleukin-8 by cultured endothelial cells in response to Borrelia burgdorferi occurs independently of secreted IL-1 and tumor necrosis factor alpha and is required for subsequent transendothelial migration of neutrophils. Infect. Immun. 65:1217-1222.

Calderwood, S. B., and J. J. Mekalanos. 1988. Confirmation of the Fur operator site by insertion of a synthetic oligonucleotide into an operon fusion plasmid. J. Bacteriol. 170:1015-1017. 
Campbell, J. A., W. S. Paul, M. E. Schriefer, R. B. Craven, K. E. Robbins, and D. T. Dennis. 1995. Epidemiologic and diagnostic studies of patients with suspected early Lyme disease, Missouri, 1990-1993. J. Infect. Dis. 172:470-480.

Carlyon, J. A., C. LaVoie, S.-Y. Sung, and R. T. Marconi. 1998. Analysis of the organization of multicopy linear- and circular-plasmid-carried open reading frames in Borrelia burgdorferi sensu lato isolates. Infect. Immun. 66:1149-1158.

Carreiro, M. M., D. C. Laux, and D. R. Nelson. 1990. Characterization of the heat shock response and identification of heat shock antigens of Borrelia burgdorferi. Infect. Immun. 58:2186-2191.

Carroll, J. A., R. M. Cordova, and C. F. Garon. 2000. Identification of 11 pH-regulated genes in Borrelia burgdorferi localizing to linear plasmids. Infect. Immun. 68:66776684.

Carroll, J. A., D. W. Dorward, and F. C. Gherardini. 1996. Identification of a transferrinbinding protein from Borrelia burgdorferi. Infect. Immun. 64:2911-2916.

Carroll, J. A., C. F. Garon, and T. G. Schwan. 1999. Effects of environmental pH on membrane proteins in Borrelia burgdorferi. Infect. Immun. 67:3181-3187.

Carroll, J. A., and F. C. Gherardini. 1996. Membrane protein variations associated with in vitro passage of Borrelia burgdorferi. Infect. Immun. 64:392-398.

Casjens, S., N. Palmer, R. vanVugt, W. M. Huang, B. Stevenson, P. Rosa, R. Lathigra, G. Sutton, J. Peterson, R. J. Dodson, D. Haft, E. Hickey, M. Gwinn, O. White, and C. M. Fraser. 2000. A bacterial genome in flux: the twelve linear and nine circular extrachromosomal DNAs in an infectious isolate of the Lyme disease spirochete Borrelia burgdorferi. Mol. Microbiol. 35:490-516.

Casjens, S. R. van Vugt, K. Tilly, P. A. Rosa, and B. Stevenson. 1997. Homology throughout the multiple 32-kilobase circular plasmids present in Lyme disease spirochetes. J. Bacteriol. 179:217-227.

Centers for Disease Control. 1999. Recommendation for the use of Lyme disease vaccine. Morbid. Mortal. Weekly Rep. 48:11-13. 
Centers for Disease Control. 2001. Summary of notifiable diseases, United States, 2000. Morbid. Mortal. Weekly Rep. 50:76-77.

Chamberlin, M. J. 1974. The selectivity of transcription. Annu. Rev. Biochem. 43:721-725.

Charon, N. W., E. P. Greenberg, M. B. Koopman, and R. J. Limberger. 1992. Spirochete chemotaxis, motility, and the structure of the spirochetal periplasmic flagella. Res. Microbiol. 143:597-603.

Chen, L., L. P. James, and J. D. Helmann. 1993. Metalloregulation in Bacillus subtilis: isolation and characterization of two genes differentially repressed by metal ions. J. Bacteriol. 175:5428-5437.

Chen, L., L. Keramati, and J. D. Helmann. 1995. Coordinate regulation of Bacillus subtilis peroxide stress genes by hydrogen peroxide and metal ions. Proc. Natl. Acad. Sci. USA 92:8190-8194.

Chen, X., T. Brown, and P. C. Tai. 1998. Identification and characterization of proteaseresistant SecA fragments: SecA has two membrane-integral forms. J. Bacteriol. 180:527-537.

Chen, Y., and E. M. Gregory. 1991. In vivo metal substitution in Bacteroides fragilis superoxide dismutase. Free Rad. Res. Comm. 12:313-318.

Clover, J. R., and R. S. Lane. 1995. Evidence implicating nymphal Ixodes pacificus in the epidemiology of Lyme disease in California. Am. J. Trop. Med. Hyg. 53:237-240.

Cluss, R. G., and J. T. Boothby. 1990. Thermoregulation of protein synthesis in Borrelia burgdorferi. Infect. Immun. 58:1038-1042.

Coleman, J. L., and J. L. Benach. 1999. Use of the plasminogen activation system by microorganisms. J. Lab. Clin. Med. 134:567-576.

Coleman, J. L., J. A. Gebbia, and J. L. Benach. 2001. Borrelia burgdorferi and other bacterial products induce expression and release of the urokinase receptor (CD87). J. Immunol. 166:473-480. 
Coleman, J. L., J. A. Gebbia, J. Piesman, J. L. Degen, T. H. Bugge, and J. L. Benach. 1997. Plasminogen is required for efficient dissemination of Borrelia burgdorferi in ticks and for enhancement of spirochetemia in mice. Cell 89:1111-1119.

Coleman, J. L., E. J. Roemer, and J. L. Benach. 1999. Plasmin-coated Borrelia burgdorferi degrades soluble and insoluble components of the mammalian extracellular matrix. Infect. Immun. 67:3929-3936.

Coleman, J. L., T. J. Sellati, J. E. Testa, R. R. Kew, M. B. Furie, and J. L. Benach. 1995. Borrelia burgdorferi binds plasminogen, resulting in enhanced penetration of endothelial monolayers. Infect. Immun. 63:2478-84.

Compan, I., and D. Touati. 1993. Interaction of six global transcription regulators in expression of manganese superoxide dismutase in Escherichia coli K-12. J. Bacteriol. 175:1687-1696.

Cowing, D. W., J. C. A. Bardwell, E. A. Craig, C. Woolford, R. W. Hendrix, and C. A. Gross. 1985. Consensus sequence for Escherichia coli heat shock gene promoters. Proc. Natl. Acad. Sci. USA 82:2679-2683.

Cozzarelli, N. R. 1980. DNA gyrase and the supercoiling of DNA. Science 207:953-960.

Damman, C. J., C. H. Eggers, D. S. Samuels, and D. B. Oliver. 2000. Characterization of Borrelia burgdorferi BlyA and BlyB proteins: a prophage-encoded holin-like system. J. Bacteriol. 182:6791-6797.

Davis, B. J. 1964. Disc gel electrophoresis. II. Method and application to human serum proteins. Ann. N. Y. Acad. Sci. 121:404-427.

Dawson, J. E., C. K. Warner, S. Standeart, and J. G. Olson. 1996. The interface between research and the diagnosis of an emerging tick-borne disease, human ehrlichiosis, due to Ehrlichia chaffeensis. Arch. Intern. Med. 156:137-142.

deLorenzo, V., S. Wee, M. Herrero, and J. B. Neilands. 1987. Operator sequences of the aerobactin operon of plasmid ColV-K30 binding the ferric uptake regulation (Fur) repressor. J. Bacteriol. 169:2624-2630. 
Dobrikova, E. Y., J. Bugrysheva, and F. C. Cabello. 2001. Two independent transcriptional units control the complex and simultaneous expression of the bmp paralogous chromosomal gene family in Borrelia burgdorferi. Mol. Microbiol. 39:370-378.

Doi, R. H., and L. F. Wang. 1986. Multiple procaryotic ribonucleic acid polymerase sigma factors. Microbiol. Rev. 50:227-243.

Dorward, D. W., V. E. Tamplin, J. A. Carroll, and F. C. Gherardini. 1994. Detection and possible identification of a transferrin receptor in the outer sheath of Borrelia burgdorferi. Proceedings of VI International Conference on Lyme Borreliosis, Bologna, Italy.

Dowds, A. C. 1994. Oxidative stress response in Bacillus subtilis. FEMS Microbiol. Lett.. 124:255-264.

Dressler, F., J. A. Whalen, B. N. Reinhardt, and A. C. Steere. 1993. Western blot in serodiagnosis of Lyme disease. J. Infect. Dis. 167:392-400.

Duke, J. B. 1978. The biology of oxygen radicals. Science 201:875-880.

Dumler, J. S., and J. S. Bakken. 1995. Ehrlichial diseases of humans: emerging tick-borne infections. Clin. Infect. Dis. 5:1102-1110.

Economou, A., J. A. Pogliano, J. Beckwith, D. B. Oliver, and W. Wickner. 1995. SecA membrane cycling at SecYEG is driven by distinct ATP binding and hydrolysis events and is regulated by SecD and SecF. Cell 83:1171-1181.

Edlow, J. A. 1999. Lyme disease and related tick-borne illnesses. Ann. Emerg. Med. 33:680693.

Eggenberger, E. R. 1993. Facial palsy in Lyme disease. New Engl. J. Med. 328:1571-1576.

Eggers, C. H., and D. S. Samuels. 1999. Molecular evidence for a new bacteriophage of Borrelia burgdorferi. J. Bacteriol. 181:7308-7313. 
Elias, A. F., J. L. Bono, J. A. Carroll, P. Stewart, K. Tilly, and P. Rosa. 2000. Altered stationary-phase response in a Borrelia burgdorferi rpoS mutant. J. Bacteriol. 182:2909-2918.

Engstrom, S. M., E. Shoop, and R. C. Johnson. 1995. Immunoblot interpretation criteria for serodiagnosis of early Lyme disease. J. Clin. Microbiol. 33:419-427.

Erdile, L. F., M. A. Brandt, D. J. Warakomski, G. J. Westrack, A. Sadziene, and A. G. Barbour. 1993. Role of attached lipid in immunogenicity of Borrelia burgdorferi OspA. Infect. Immun. 61:81-90.

Escolar, L., J. Perez-Martin, and V. deLorenzo. 1998. Binding of the Fur (ferric uptake regulator) repressor of Escherichia coli to arrays of the GATAAT sequence. J. Mol. Biol. 283:537-547.

Escolar, L., J. Perez-Martin, and V. deLorenzo. 1999. Opening the iron box: transcriptional metalloregulation by the Fur protein (ferric uptake regulator). J. Bacteriol. 181:6223-6229.

Farr, S. B., and T. Kogoma. 1991. Oxidative stress responses in Escherichia coli and Salmonella typhimurium. Microbiol. Rev. 55:561-585.

Farr, S. B., D. O. Natvig, and T. Kogoma. 1985. Toxicity and mutagenicity of plumbagin and the induction of a possible new DNA repair pathway in Escherichia coli. J. Bacteriol. 164:1309-1316.

Ferdows, M. S., and A. G. Barbour. 1989. Megabase-sized linear DNA in the bacterium Borrelia burgdorferi, the Lyme disease agent. Proc. Natl. Acad. Sci. USA 86:59695973.

Fernandez, L. A., and J. Berenguer. 2000. Secretion and assembly of regular surface structures in Gram-negative bacteria. FEMS Microbiol. Lett. 24:21-44.

Fikrig, E., S. W. Barthold, and M. Chen. 1996. Protective antibodies in murine Lyme disease arise independently of CD40 ligand. J. Immunol. 157:1-6 
Fikrig, E., S. R. Telford, and S. W. Barthold. 1992. Elimination of Borrelia burgdorferi from vector ticks feeding on Osp-A immunized mice. Proc. Natl. Acad. Sci. USA 89:5418-5421.

Fingerle, V., U. Hauser, G. Liegl, B. B. Petko, V. Preac-Mursic, and B. Wilske. 1995. Expression of outer surface proteins A and C of Borrelia burgdorferi in Ixodes scapularis. J. Clin. Microbiol. 33:1867-1869.

Fraser, C. M., S. Casjens, W. M. Huang, G. G. Sutton, R. Clayton, R. Lathigra, O. White, K. A. Ketchum, R. Dodson, E. K. Hickey, M. Gwinn, B. Dougherty, J.-F. Tomb, R. D. Fleischmann, D. Richardson, J. Peterson, A. R. Kerlavage, J. Quackenbush, S. Salzberg, M. Hanson, R. V. Vugt, N. Palmer, M. D. Adams, J. Gocayne, J. Weidman, T. Utterback, L. Watthey, L. McDonald, P. Artiach, C. Bowman, S. Garland, C. Fujii, M. D. Cotton, K. Horst, K. Roberts, B. Hatch, H. O. Smith, and J. C. Venter. 1997. Genomic sequence of a Lyme disease spirochete, Borrelia burgdorferi. Nature 390:580-586.

Fraser, C. M., J. D. Gocayne, O. White, M. D. Adams, R. A. Clayton, R. D. Fleischmann, C. J. Bult, A. R. Kerlavage, G. G. Sutton, J. M. Kelley, J. L. Fritchman, J. F. Weidman, K. V. Small, M. Sandusky, J. L. Fuhrmann, D. T. Nguyen, T. Utterback, D. M. Saudek, C. A. Phillips, J. M. Merrick, J. Tomb, B. A. Dougherty, K. F. Bott, P. C. Hu, T. S. Lucier, S. N. Peterson, H. O. Smith, and J. C. Venter. 1995. The minimal gene complement of Mycoplasma genitalium. Science 270:397-403.

Fraser, C. M., S. J. Norris, G. M. Weinstock, O. White, G. G. Sutton, R. Dodson, M. Gwinn, E. K. Hickey, R. Clayton, K. A. Ketchum, E. Sodergren, J. M. Hardham, M. P. McLeod, S. Salzberg, J. Peterson, H. Khalak, D. Richardson, J. K. Howell, M. Chidambaram, T. Utterback, L. McDonald, P. Artiach, C. Bowman, M. D. Cotton, and J. C. Venter. 1998. Complete genome sequence of Treponema pallidum, the syphilis spirochete. Science 281:375-388.

Frechon, D., and E. L. Cam. 1994. Fur (ferric uptake regulation) protein interaction with target DNA: comparison of gel retardation, footprinting and electron microscopy analyses. Biochem. Biophys. Res. Commun. 201:346-355.

Fridovich, I. 1986. Superoxide dismutases. Adv. Enzymol. 58:62-97.

Fridovich, I. 1997. Superoxide anion radical $\left(\mathrm{O}_{2}^{-}\right)$, superoxide dismutases, and related matters. J. Biol. Chem. 272:18515-18517. 
Fuchs, H., R. Wallich, M. M. Simon, and M. D. Kramer. 1994. The outer surface protein A of the spirochete Borrelia burgdorferi is a plasmin(ogen) receptor. Proc. Natl. Acad. Sci. USA 91:12594-12598.

Fukunaga M., K. Okada, M. Nakao, T. Konishi, and Y. Sato. 1996. Phylogenetic analysis of Borrelia species based on flagellin gene sequences and its application for molecular typing of Lyme disease borreliae. Int. J. Syst. Bacteriol. 46:898-905.

Gaballa, A., and J. D. Helmann. 1998. Identification of a zinc-specific metalloregulatory protein, Zur, controlling zinc transport operons in Bacillus subtilis. J. Bacteriol. 180:5815-5821.

Gabbianelli, R., A. Battistoni, F. Polizio, T. Carri, A. DeMartino, B. Meier, A. Desideri, and G. Rotilio. 1995. Metal uptake of recombinant cambialistic superoxide dismutase from Propionibacterium shermanii is affected by growth conditions of host Escherichia coli cells. Biochem. Biophys. Res. Commun. 216:841-847.

Gamer, J., H. Bujard, and B. Bujau. 1992. Physical interaction between heat shock proteins DnaK, DnaJ and GrpE chaperones regulates activity of the Escherichia coli heat shock transcription factor $\sigma^{32}$. EMBO J. 15:607-617.

Garcia, R., L. Gusmani, R. Murgia, C. Guarnaccia, M. Cinco, and G. Rottini. 1998. Elastase is the only human neutrophil granule protein that alone is responsible for in vitro killing of Borrelia burgdorferi. Infect. Immun. 66:1408-1412.

Garin, C., and C. Bujadoux. 1922. Paralysie par les Tiques. J. Med. Lyon 71:765-767.

Ge, Y. and N. W. Charon. 1997. An unexpected flaA homolog is present and expressed in Borrelia burgdorferi. J. Bacteriol. 179:552-556.

Ge, Y., I. G. Old, I. S. Girons, and N. W. Charon. 1997. The flgK motility operon of Borrelia burgdorferi is initiated by a $\sigma^{70}$-like promoter. Microbiol. 143:1681-1690.

Gegenheimer, P., and D. Apirion. 1981. Processing of procaryotic ribonucleic acid. Microbiol. Rev. 45:502-541. 
Georgilis, K., M. Peacocke, and M. S. Klempner. 1992. Fibroblasts protect the Lyme disease spirochete, Borrelia burgdorferi, from ceftriaxone in vitro. J. Infect. Dis. 166:440-444.

Gething, M. J., and J. Sambrook. 1992. Protein folding in the cell. Nature 355:33-45.

Gomez, A. A. Mve-Obiang, B. Vray, W. Rudnicka, I. C. Shamputa, F. Porteals, W. M. Meyers. P. A. Fonteyne, and L. Realini. 2001. Detection of phospholipase C in non-tuberculous mycobacteria and its possible role in hemolytic activity. J. Clin. Microbiol. 39:1396-1401.

Gonzalez de Peredo, A., C. Staint-Pierre, A. Adriat., L. Jacquamet, J. M. Latour, I. Michaud-Soret, and E. Forest. 1999. Identification of the two zinc-bound cysteines in the ferric uptake regulation protein from Escherichia coli: chemical modification and mass spectrometry analysis. Biochemistry 38:8582-8589.

Grandsaerd, J. J., and A. A. Meulenbroeks. 2000. Lyme borreliosis as a cause of facial palsy during pregnancy. Eur. J. Obstet. Gynecol. Reprod. Biol. 91:99-101.

Gribskov, M., and R. R. Burgess. 1986. Sigma factors from Escherichia coli, Bacillus subtilis, phage SP01, and phage T4 are homologous proteins. Nucleic Acids Res. 14:6745-6763.

Griggs, D. W., and J. Konisky. 1989. Mechanism for iron-regulated transcription of the Escherichia coli cir gene: metal-dependent binding of Fur protein to the promoters. J. Bacteriol. 171:1048-1054.

Groote, M. A., U. A. Ochsner, M. U. Shiloh, C. Nathan, J. M. McCord, M. C. Dinauer, S. J. Libby, A. Vazquez-Torres, Y. Xu, and F. C. Fang. 1997. Periplasmic superoxide dismutase protects Salmonella from products of phagocyte NADPHoxidase and nitric oxide synthase. Proc. Natl. Acad. Sci. USA 94:13997-14001.

Grossman, A. D., J. W. Erickson, and C. A. Gross. 1984. The htpR gene product of E. coli is a sigma factor for heat-shock promoters. Cell 38:383-390.

Guina, T., D. Helfet-Hilliker, V. Ramamurthy, and D. Oliver. 1998. Sequence and phylogenetic analysis of the Borrelia burgdorferi secA gene. Biochim. Biophys. Acta 1371:24-30. 
Guina, T., and D. B. Oliver. 1997. Cloning and analysis of a Borrelia burgdorferi membrane-interactive protein exhibiting hemolytic activity. Mol. Microbiol. 24:12011213.

Gunsalus, R. P., and S. J. Park. 1994. Aerobic-anaerobic gene regulation in Escherichia coli control by the ArcAB and Fnr regulons. Res. Microbiol. 145:437-450.

Guo, B. P., E. L. Brown, D. W. Dorward, L. C. Rosenberg, and M. Hook. 1998. Decorinbinding adhesin from Borrelia burgdorferi. Mol. Microbiol. 30:711-723.

Guo, B. P., S. J. Norris, L. C. Rosenberg, and M. Hook. 1995. Adherence of Borrelia burgdorferi to the proteoglycan decorin. Infect. Immun. 63:3467-3472.

Haake, D. A. 2000. Spirochetal lipoproteins and pathogenesis. Microbiol. 146:1491-1504.

Hahn, J. S., S. Y. Oh, and J. H. Roe. 2000. Regulation of the furA and catC operon, encoding a ferric uptake regulator homologue and catalase-peroxidase, respectively, in Streptomyces coelicolor A3(2). J. Bacteriol. 182:3767-3774.

Harley, C. B., and R. P. Reynolds. 1987. Analysis of E. coli promoter sequences. Nucleic Acids Res. 15:2343-2350.

Hawley, D. K., and R. McClure. 1983. Compilation and analysis of Escherichia coli promoter DNA sequences. Nucleic Acids Res. 11:2237-2255.

He, B., Y. Choi, and H. Zalkin. 1993. Regulation of Escherichia coli glnA, prsA and speA by the purine repressor. J. Bacteriol. 175:3598-3606.

Hellman, U., C. Wernstedt, J. Gonez, and C. H. Heldin. 1995. Improvement of an in-gel digestion procedure for the micropreparation of internal protein fragments for amino acid sequencing. Anal. Biochem. 224:451-455.

Helmann, J. D., and M. J. Chamberlin. 1988. Structure and function of bacterial sigma factors. Annu. Rev. Biochem. 57:839-872. 
Herendeen, S. L., R. A. VanBogelen, and F. C. Neidhardt. 1979. Levels of major proteins of Escherichia coli during growth at different temperatures. J. Bacteriol. 139:185194.

Hinnebusch, J., and A. G. Barbour. 1992. Linear- and circular-plasmid copy numbers in Borrelia burgdorferi. J. Bacteriol. 174:5251-5257.

Holt, S. C. 1978. Anatomy and chemistry of spirochetes. Microbiol. Rev. 42:114-160.

Horsburgh, M. J., E. Ingham, and S. J. Foster. 2001. In Staphylococcus aureus, Fur is an interactive regulator with PerR, contributes to virulence, and is necessary for oxidative stress resistance through positive regulation of catalase and iron homeostasis. J. Bacteriol. 183:468-475.

Howe, T. R., F. W. Laquier, and A. G. Barbour. 1986. Organization of genes encoding two outer membrane proteins of the Lyme disease agent Borrelia burgdorferi within a single transcriptional unit. Infect. Immun. 54:207-212.

Hu, L. T., and M. S. Klempner. 2001. Update on the prevention, diagnosis, and treatment of Lyme disease. Adv. Intern. Med. 46:247-275.

Hu, L. T., G. Perides, R. Noring, and M. S. Klempner. 1995. Binding of human plasminogen to Borrelia burgdorferi. Infect. Immun. 63:3491-3496.

Hubalek, Z., and J. Halouzka. 1997. Distribution of B. burgdorferi sensu lato genomic groups in Europe, a review. Eur. J. Epidemiol. 13:951-957.

\section{Huisman, O., M. Faelen, D. Girard, A. Jaffe, A. Toussaint, and J. Rouviere-Yaniv.} 1989. Multiple defects in Escherichia coli mutant lacking HU protein. J. Bacteriol. 171:3704-3712.

Humair, P., and L. Gern. 2000. The wild hidden face of Lyme borreliosis in Europe. Microbes Infect. 2:915-922.

Hunt, T. P., and B. Magasanik. 1985. Transcription of $g \ln A$ by purified Escherichia coli components: core RNA polymerase and the products of $g \ln F, g \ln G$, and $g \ln L$. Proc. Natl. Acad. Sci. USA 82:8453-8457. 
Imlay, J. A., S. M. Chin, and S. Linn. 1988. Toxic DNA damage by hydrogen peroxide through the Fenton reaction in vivo and in vitro. Science 48:640-642.

Imlay, J. A., and S. Linn. 1986. Bimodal pattern of killing of DNA-repair-defective or anoxically grown Escherichia coli by hydrogen peroxide. J. Bacteriol. 166:797-799.

Isaacs, R. D. 1994. Borrelia burgdorferi bind to epithelial cell proteoglycans. J. Clin. Invest. 93:809-819.

Isogai, H., K. Kimura, S. Hayashi, T. Kubota, N. Fujii, T. Nishikawa, S. Kotake, and E. Isogai. 1997. Levels of endogenous interleukin-1, interleukin-6, and tumor necrosis factor in congenic mice infected with Borrelia garinii. Microbiol. Immunol. 41:427430.

Iuchi, S., and L. Weiner. 1996. Cellular and molecular physiology of Escherichia coli in the adaptation to aerobic environments. J. Biochem. 120:1055-1063.

Jacquamet, L., D. Aberdam, A. Adriat, J. L. Hazemann, J. M. Latour, and I. MichaudSoret. 1998. X-ray absorption spectroscopy of a new zinc site in the Fur protein from Escherichia coli. Biochemistry 37:2564-2571.

Jaeger, J. A., D. H. Turner, and M. Zucker. 1990. Predicting optimal and suboptimal secondary structures for RNA. Methods Enzymol. 183:281-306.

Jianhui, W., T. Masuzawa, T. Komikado, and Y. Yanagihara. 1997. Consensus sequence of the genes encoding the major outer surface proteins (OspA and OspB) of Borrelia garinii isolate. Microbiol. Immunol. 41:83-91.

Johnson, R. C., C. Kodner, and M. Russell. 1986. Active immunization of hamsters against experimental infection with Borrelia burgdorferi. Infect. Immun. 61:27742777.

Jonsson, M., L. Noppa, A. G. Barbour, and S. Bergstrom. 1992. Heterogeneity of outer membrane proteins in Borrelia burgdorferi: comparison of osp operons of three isolates of different geographic origins. Infect. Immun. 60:1845-1853.

Jovanovic, T., C. Ascenso, K. R. Hazlett, R. Sikkink, C. Krebs, R. Litwiller, L. M. Benson, I. Moura, J. J. Moura, J. D. Radolf, B. H. Huynh, S. Naylor, and F. 
Rusnak. 2000. Neelaredoxin, an iron-binding protein from the syphilis spirochete, Treponema pallidum, is a superoxide reductase. J. Biol. Chem. 275:28439-28448.

Jungblat, P. R., Grabher, G., and G. Stoffler. 1999. Comprehensive detection of immunorelevant Borrelia garinii antigens by two-dimensional electrophoresis. Electrophoresis 20:3611-3622.

Kanemori, M., K. Nichihara, H. Yanagi, and T. Yura. 1994. Induction of heat shock proteins by abnormal proteins results from stabilization and not increased synthesis of $\sigma^{32}$ in Escherichia coli. J. Bacteriol. 176:5648-5653.

Kaplan, R. F., and L. Jones-Woodward. 1997. Lyme encephalopathy: a neuropsychological perspective. Seminars Neurol. 17:31-37.

Kesavalu, L., S. C. Holt, and J. L. Ebersole. 1999. Environmental modulation of oral treponeme virulence in a murine model. Infect. Immun. 67:2783-2789.

Kindstrand, E., B. Y. Nilsson, A. Hovmark, I. Nennesmo, R. Pirskanen, and E. Asbrink. 1997. Peripheral neuropathy in acrodermatitis chronica atrophicans - late Borrelia manifestation. Acta Neurol. Scand. 95:47-52.

Kindstrand, E., B. Y. Nilsson, A. Hovmark, I. Nennesmo, R. Pirskanen, G. Solders, and E. Asbrink. 2000. Polyneuropathy in late Lyme borreliosis-a clinical, neurophysiological and morphological description. Acta Neurol Scand. 101:47-52.

Kirby, T., J. Blum, I. Kahane, and I. Fridovich. 1980. Distinguishing between Mncontaining and Fe-containing superoxide dismutases in crude extracts of cells. Arch. Biochem. Biophys. 201:551-555.

Klaviter, E. C., and R. C. Johnson. 1969. Isolation of the outer envelope, chemical components, and ultrastructure of Borrelia hermsi grown in vitro. Acta Trop. 36:123131.

Klein, J., G. Stanek, R. Bittner, R. Horvat, C. Holzinger, and D. Glogar. 1991. Lyme borreliosis as a cause of myocarditis and heart muscle disease. Eur. Heart J. 12(Suppl D):73-75. 
Klempner, M. S., R. Noring, M. P. Epstein, B. McCloud, R. Hu, S. A. Limentani, and R. Rogers. 1995. Binding of human plasminogen and urokinase-type plasminogen activator to the Lyme disease spirochete, Borrelia burgdorferi. J. Infect. Dis. 171:1258-1265.

Knight, S. W., B. J. Kimmel, C. H. Eggers, and D. S. Samuels. 2000. Disruption of the Borrelia burgdorferi gac gene, encoding the naturally synthesized Gyr-A Cterminal domain. J. Bacteriol. 182:2048-2051.

Knight, S. W., and D. S. Samuels. 1999. Natural synthesis of a DNA-binding protein from the C-terminal domain of DNA gyrase A in Borrelia burgdorferi. EMBO J. 18:48754881.

Kolter, R. 1992. Life and death in stationary phase. ASM News 58:75-79.

Kroll, J. S., P. R. Langford, J. R. Saah, and F. M. Loynds. 1993. Molecular and genetic characterization of superoxide dismutase in Haemophilus influenzae type b. Mol. Microbiol. 10:839-848.

Kroll, J. S., P. R. Langford, K. E. Wilks, and A. D. Keil. 1995. Bacterial (Cu,Zn)superoxide dismutase: phylogenetically distinct from the eukaryotic enzyme, and not so rare after all! Microbiol. 141:2271-2279.

Kurtenbach, K., M. Peacy, S. G. Rijpkema, A. N. Hoodless, P. A. Nuttall, and S. E. Randolph. 1998. Differential transmission of the genospecies of Borrelia burgdorferi sensu lato by game birds and small rodents in England. Appl. Environ. Microbiol. 64:1169-1174.

Kusunose, E., K. Ichihara, Y. Noda, and M. Kusunose. 1976. Superoxide dismutase of Mycobacterium tuberculosis. J. Biochem. 80:1343-1352.

Labandeira-Rey, M., and J. T. Skare. 2001. Decreased infectivity in Borrelia burgdorferi strain B31 is associated with loss of linear plasmid 25 or 29-1. Infect. Immun. 69:446-455.

Laemmli, U. K. 1970. Cleavage of structural proteins during the assembly of the head of bacteriophage T4. Nature 227:680-685. 
Landick, R., V. Vaughn, E. T. Lau, R. A. V. Bogelen, J. W. Erickson, and F. C. Neidhardt. 1984. Nucleotide sequence of the heat shock regulatory gene of E. coli suggests its protein product may be a transcription factor. Cell 38:175-182.

Lane, R. S. 1990. Susceptibility of the western fence lizard (Sceloporus occidentalis) to the Lyme borreliosis spirochete (Borrelia burgdorferi). Am. J. Trop. Med. Hyg. 42:7582.

Lane, R. S., and J. E. Loye. 1991. Lyme disease in California: interrelationship of Ixodid ticks, rodents and Borrelia burgdorferi. J. Med. Entomol. 28:719-725.

Lane, R. S., and G. B. Quistad. 1998. Borreliacidal factor in the blood of the western fence lizard (Sceloporus occidentalis). J. Parasitol. 84:29-34.

Lange, R., and R. Hengge-Aronis. 1991. Identification of a central regulator of stationaryphase gene expression in Escherichia coli. Mol. Microbiol. 5:49-59.

Lee, Y. H., R. K. Deka, M. V. Norgard, J. D. Radolf, and C. A. Hasemann. 1999. Treponema pallidum TroA is a periplasmic zinc-binding protein with a helical backbone. Nature Struct. Biol. 6:628-633.

Leong, J. M., H. Wang, L. Magoun, J. A. Field, P. E. Morrissey, D. Robbins, J. B. Tatro, J. Coburn, and N. Parveen. 1998. Different classes of proteoglycans contribute to the attachment of Borrelia burgdorferi to cultured endothelial and brain cells. Infect. Immun. 66:994-999.

Leoni, L., N. Orsi, V. deLorenzo, and P. Visca. 2000. Functional analysis of PvdS, an iron starvation sigma factor of Pseudomonas aeruginosa. J. Bacteriol. 182:1481-1491.

Lesser, R. L. 1995. Ocular manifestations of Lyme disease. Am. J. Med. 98:60S-62S.

Levin, M. L., and D. Fish. 2000. Acquisition of coinfection and simultaneous transmission of Borrelia burgdorferi and Ehrlichia phagocytophilia by Ixodes scapularis ticks. Infect. Immun. 68:2183-2186.

Li, M., T. Masuzawa, N. Takada, F. Iabigura, H. Fugita, A. Iwaki, H. Wang, J. Wang, M. Kawabata, and Y. Yanagihara. 1998. Lyme disease Borrelia species in 
northeastern China resemble those isolated from far eastern Russia and Japan. Appl. Environ. Microbiol. 64:2705-2509.

Lill, R., K. Cunningham, L. A. Brundage, K. Ito, D. Oliver, and W. Wickner. 1989. SecA protein hydrolyzes ATP and is an essential component of the protein translocation ATPase of Escherichia coli. EMBO J. 8:961-966.

Lin, J. J., and A. Sancar. 1989. A new mechanism for repairing oxidative damage to DNA: (a)Bc exinuclease removes AP sites and thymine glycols from DNA. Biochemistry. 28:7979-7984.

Lindsay, J. A., and S. J. Foster. 2001. Zur: a $\mathrm{Zn}^{2+}$ responsive element of Staphylococcus aureus. Microbiol. 147:1259-1266.

Livermore, B. P., R. F. Bey, and R. C. Johnson. 1978. Lipid metabolism of Borrelia hermsi. Infect. Immun. 20:215-220.

Loewen, P. C., J. Switala, M. R. Mulvey, and T. Thorvaldson. 1991. Catalases HPI and HPII in Escherichia coli are induced independently. Arch. Biochem. Biophys. 243:144-149.

Loewen, P. C., and B. L. Triggs. 1984. Genetic mapping of katF, a locus that with katE affects the synthesis of a second catalase species in Escherichia coli. J. Bacteriol. 160:668-675.

Lombard, M., D. Touati, M. Fontecave, and V. Niviere. 2000. Superoxide reductase as a unique defense system against superoxide stress in the microaerophile Treponema pallidum. J. Biol. Chem. 275:27021-27026.

Ma, Y., K. P. Seiler, K. F. Tai, L. Yang, M. Woods, and J. J. Weis. 1994. Outer surface lipoproteins of Borrelia burgdorferi stimulate nitric oxide production by the cytokineinducible pathway. Infect. Immun. 62:3663-3667.

Ma, Y., A. Sturrock, and J. J. Weis. 1991. Intracellular localization of Borrelia burgdorferi within human endothelial cells. Infect. Immun. 59:671-678. 
Ma, Y. A., and J. J. Weis. 1993. Borrelia burgdorferi outer surface lipoproteins OspA and OspB possess B-cell mitogenic and cytokine-stimulatory properties. Infect. Immun. 61:3843-3853.

Magnarelli, L. A., K. C. Stafford III, J. W. Ijdo, and E. Fikrig. 1995. Coexistence of antibodies to tick-borne pathogens of babesiosis, ehrlichiosis, and Lyme disease in human sera. J. Clin. Microbiol. 33:3054-3057.

Mangel, W. F., and M. J. Chamberlin. 1974. Studies of ribonucleic acid chain initiation by Escherichia coli ribonucleic acid polymerase bound to T7 deoxyribonucleic acid. I. An assay for the rate and extent of ribonucleic acid chain initiation. J. Biol. Chem. 249:2995-3001.

Manweiler, S. A., R. S. Lane, and C. H. Tempelis. 1992. The western fence lizard, Sceloporus occidentalis: evidence of field exposure to Borrelia burgdorferi in relation to infestation by Ixodes pacificus. Am. J. Trop. Med. Hyg. 47:328-336.

Marconi, R. T., M. E. Konkel, and C. F. Garon. 1993a. Variability of osp genes and gene products among species of Lyme disease spirochetes. Infect. Immun. 61:2611-2617.

Marconi, R. T., D. S. Samuels, and C. F. Garon. 1993b. Transcriptional analyses and mapping of the $\operatorname{spC}_{\mathrm{g}}$ gene in Lyme disease spirochetes. J. Bacteriol. 175:926-932.

Marconi, R. T., D. S. Samuels, R. K. Landry, and C. F. Garon. 1994. Analysis of the distribution and molecular heterogeneity of the $\operatorname{sip} D$ gene among the Lyme disease spirochetes: evidence for lateral gene exchange. J. Bacteriol. 176:4572-4582.

Marconi, R. T., D. S. Samuels, T. G. Schwan, and C. F. Garon. 1993c. Identification of a protein in several Borrelia species which is related to OspC of the Lyme disease spirochetes. J. Clin. Microbiol. 31:2577-2583.

Marconi, R. T., S. Y. Sung, C. A. N. Hughes, and J. A. Carlyon. 1996. Molecular and evolutionary analyses of a variable series of genes in Borrelia burgdorferi that are related to $o s p E$ and $o s p F$, constitute a gene family, and share a common upstream homology box. J. Bacteriol. 178:5615-5626.

Masters, E. J., and H. D. Donnell. 1995. Lyme and/or Lyme-like disease in Missouri. Missouri Med. 92:346-353. 
Mather, T. N., M. C. Nicholson, R. Hu., and N. J. Miller. 1996. Entomological correlates of Babesia microti prevalence in an area where Ixodes scapularis is endemic. J. Med. Entomol. 33:866-870.

McCord, J. M., and I. Fridovich. 1969. Superoxide dismutase: an enzyme function for erythrocuprein (hemocuprein). J. Biol. Chem. 244:6049-6055.

McKenna, W. R., P. A. Mickelson, P. F. Sparling, and D. W. Dyer. 1988. Iron uptake from lactoferrin and transferrin by Neisseria gonorrhoeae. Infect. Immun. 56:785:791.

McNicholas, P., T. Rajapandi, and D. Oliver. 1995. SecA proteins of Bacillus subtilis and Escherichia coli possess homologous amino-terminal ATP-binding domains regulating integration into the plasma membrane. J. Bacteriol. 177:7231-7237.

Meier, B., D. Barra, F. Bossa, L. Calabrese, and G. Rotilio. 1982. Synthesis of either Feor Mn-superoxide dismutase with an apparently identical protein moiety by an anaerobic bacterium dependent on the metal supplied. J. Biol. Chem. 257:1397713980.

Merden, H. A., and L. A. Durden. 2000. A state-by-state survey of ticks recorded from humans in the United States. J. Vector Ecol. 25:102-113.

Michiels, C., M. Raes, O. Toussaint, and J. Remacle. 1994. Importance of Se-glutathione peroxidase, catalase, and $\mathrm{Cu} / \mathrm{Zn}-\mathrm{SOD}$ for cell survival against oxidative stress. Free Rad. Biol. Med. 17:235-248.

Missiakas, D., S. Raina, and C. Georgopoulos. 1996. Heat shock regulation, p. 481-501. In E. C. C. Lin and A. S. Lynch (ed.), Regulation of gene expression in Escherichia coli. R. G. Landes Company, Austin, Texas.

Modolell, M., U. E. Schaible, M. Rittig, and M. M. Simon. 1994. Killing of Borrelia burgdorferi by macrophages is dependent on oxygen radicals and nitric oxide and can be enhanced by antibodies to outer surface proteins of the spirochete. Immun. Lett. 40:139-146. 
Montgomery, R. R., and S. E. Malawista. 1996. Entry of Borrelia burgdorferi into macrophages in end-on and leads to degradation in lysosomes. Infect. Immun. 64:2867-2872.

Montgomery, R. R., M. H. Nathanson, and S. E. Malawista. 1993. The fate of Borrelia burgdorferi, the agent for Lyme disease, in mouse macrophages: destruction, survival, recovery. J. Immunol. 150:909-915.

Montgomery, R. R., M. H. Nathanson, and S. E. Malawista. 1994. Fc and non-Fcmediated phagocytosis of Borrelia burgdorferi by macrophages. J. Infect. Dis. 170:890-895.

Morret, E., and L. Segovia. 1993. The sigma 54 bacterial enhancer-binding protein family: mechanism of action and phylogenetic relationship of their functional domains. J. Bacteriol. 175:6067-6074.

Morrisey, J. H. 1981. Silver stain for proteins in polyacrylamide gels: a modified procedure with enhanced uniform sensitivity. Anal. Biochem. 117:307-310.

Morrison, T. B., J. H. Weis, and J. J. Weis. 1997. Borrelia burgdorferi outer surface protein A activates and primes human neutrophils. J. Immunol. 158:4838-4845.

Motaleb, M. A., L. Corum, J. L. Bono, A. F. Elias, P. Rosa, D. S. Samuels, and N. W. Charon. 2000. Borrelia burgdorferi periplasmic flagella have both skeletal and motility functions. Proc. Natl. Acad. Sci. USA 97:10899-10904.

Nadelman, R. B., H. W. Horowitz, T. C. Hsieh, J. M. Wu, M. E. Aguero-Rosenfeld, I. Schwartz, J. Nowakowski, S. Varde, and G. P. Wormser. 1997. Simultaneous human ehrlichiosis and Lyme borreliosis. New Eng. J. Med. 337:27-30

Nakao, M., K. Miyamoto, and M. Fukunaga. 1994. Lyme disease spirochetes in Japan: enzootic transmission cycles in birds, rodents, and Ixodes persulcatus ticks. J. Infect. Dis. 170:878-882.

Nanagara, R., P. H. Duray, and H. R. Schumacher. 1996. Ultrastructural demonstration of spirochetal antigens in synovial fluid and synovial membrane in chronic Lyme disease: possible factors contributing to persistence of organisms. Human Pathol. 27:1025-1034. 
Niederhoffer, E. C., C. M. Naranjo, K. L. Bradley, and J. A. Fee. 1990. Control of Escherichia coli superoxide dismutase ( $\operatorname{sod} A$ and $\operatorname{sod} B$ ) genes by the ferric uptake regulation (fur) locus. J Bacteriol. 172:1930-1938.

Nilsson, G., J. Belasco, S. Cohen, and A. vonGabain. 1984. Growth-rate dependent regulation of mRNA stability in Escherichia coli. Nature 312:75-77.

Norris, S. J., C. J. Carter, J. K. Howell, and A. G. Barbour. 1992. Low-passageassociated proteins of Borrelia burgdorferi B31: characterization and molecular cloning of OspD, a surface-exposed, plasmid-encoded lipoprotein. Infect. Immun. 60:4662-4672.

Nunoshiba, T., E. Hidalgo, C. F. A. Cuevas, and B. Demple. 1992. Two-stage control of an oxidative stress regulon: the Escherichia coli SoxR protein triggers redoxinducible expression of the soxS regulatory gene. J. Bacteriol. 174:6054-6060.

Obonyo, M., U. G. Munderloh, V. Fingerle, B. Wilske, and T. J. Kurtti. 1999. Borrelia burgdorferi in tick cell culture modulates expression of outer surface proteins A and $\mathrm{C}$ in response to temperature. J. Clin. Microbiol. 37:2137-2141.

Ochsner, U. A., A. I. Vasil, and M. L. Vasil. 1995. Role of the ferric uptake regulator of Pseudomonas aeruginosa in the regulation of siderophores and exotoxin A expression: purification and activity on iron-regulated promoters. J. Bacteriol. 177:7194-7201.

O'Farrell, P. A., H. M. Goodman, and P. H. O'Farrell. 1977. High resolution twodimensional electrophoresis of basic as well as acidic proteins. Cell 12:1133-1142.

Oliver, J. H. J., J. R. Owsley, H. J. Hutcheson, A. M. James, C. Chen, W. S. Iry, E. M. Dotson, and D. K. McLain. 1993. Conspecificity of the ticks Ixodes scapularis and Ixodes dammini (Acari: Ixodidae). J. Med. Entomol. 30:54-63.

Orloski, K. A., E. B. Hayes, G. L. Campbell, and D. T. Dennis. 2000. Surveillance for Lyme disease-United States, 1992-1998. Morbid. Mortal. Weekly Rep. 49:1-11. 
Osada, Y., R. Saito, and M. Tomita. 1999. Analysis of base-pairing potentials between $16 \mathrm{~S}$ rRNA and 5'UTR for translation initiation in various prokaryotes. Bioinformatics 15:578-581.

Pachner, A. R. 1995. Early disseminated Lyme disease: Lyme meningitis. Am. J. Med. 98:30S-37S.

Pachner, A. R., J. Basta, E. Delaney, and D. Hlinska. 1995. Localization of Borrelia burgdorferi in murine Lyme borreliosis by electron microscopy. Am. J. Trop. Med. Hyg. 52:128-133.

Pal, U., A. M. deSilva, R. R. Montgomery, D. Fish, J. Anguita, J. F. Anderson, Y. Lobet, and E. Fikrig. 2000. Attachment of Borrelia burgdorferi within Ixodes scapularis mediated by outer surface protein A. J. Clin. Invest. 106:561-569.

Palmer, N., C. Fraser, and S. Casjens. 2000. Distribution of twelve linear extrachromosomal DNAs in natural isolates of Lyme disease spirochetes. J. Bacteriol. 182:2476-2480.

Pancholi, V., and V. A. Fischetti. 1998. $\alpha$-Enolase, a novel strong plasmin(ogen) binding protein on the surface of pathogenic streptococci. J. Biol. Chem. 273:14503-14515.

Park, K. H., W. H. Chang, and T. G. Schwan. 1993. Identification and characterization of Lyme disease spirochetes, Borrelia burgdorferi sensu lato, isolated in Korea. J. Clin. Microbiol. 31:1831-1837.

Paster, B. J., and F. E. Dewhirst. 2000. Phylogenetic foundation of spirochetes. J. Mol. Microbiol. Biotechnol. 2:341-344.

Paster, B. J., F. E. Dewhirst, W. G. Weisburg, L. A. Tordoff, G. J. Fraser,. R. B. Hespell, T. B. Stanton, L. Zablen, L. Mandelco, and C. R. Woese. 1991. Phylogenetic analysis of the spirochetes. J. Bacteriol. 173:6101-6109.

Perides, G., R. Noring, and M. S. Klempner. 1996. Inhibition of Borrelia burgdorferibound fibrinolytic enzymes by $\alpha_{2}$-antiplasmin, PAI-1 and PAI-2. Biochem. Biophys. Res. Commun. 219:690-695. 
Peterson, P. K., C. C. Clawson, D. A. Lee, D. J. Garlich, P. G. Quie, and R. C. Johnson. 1984. Human phagocyte interactions with the Lyme disease spirochete. Infect. Immun. 46:608-611.

Piesman, J., T. N. Mather, R. J. Sinsky, and A. Spielman. 1987. Duration of tick attachment and Borrelia burgdorferi transmission. J. Clin. Microbiol. 25:557-560.

Pintor, M., C. M. Ferreiros, and M. T. Criado. 1993. Characterization of the transferriniron uptake system in Neisseria meningitidis. FEMS Microbiol. Lett. 112:159-165.

Poole, R. K., M. F. Anjum, J. Membrillo-Hernandez, S. O. Kim, M. N. Hughes, and V. Stewart. 1996. Nitric oxide, nitrite, and Fnr regulation of hmp (flavohemoglobin) gene expression in Escherichia coli K12. J. Bacteriol. 178:5487-5492.

Porcella, S. F., T. G. Popova, D. R. Akins, M. Li, J. R. Radolf, and M. V. Norgard. 1996. Borrelia burgdorferi supercoiled plasmids encode multicopy open reading frames and a lipoprotein gene family. J. Bacteriol. 178:3293-3307.

Posey, J. E., and F. C. Gherardini. 2000. Lack of a role for iron in the Lyme disease pathogen. Science 288:1651-1653.

Posey, J. E., J. M. Hardham, S. J. Norris, and F. C. Gherardini. 1999. Characterization of a manganese-dependent regulatory protein, TroR, from Treponema pallidum. Proc. Natl. Acad. Sci. USA 96:10887-10892.

Postic, D., N. M. Ras, R. S. Lane, M. Hendson, and G. Baranton. 1998. Expanded diversity among California Borrelia isolates and description of Borrelia bissettii sp. nov. (formerly Borrelia group DN127). J. Clin. Microbiol. 36:3497-3504.

Preac-Mursic, V., K. Weber, H. W. Pfister, B. Wilske, B. Gross, A. Baumann, and J. Prokop. 1989. Survival of Borrelia burgdorferi in antibiotically treated patients with Lyme borreliosis. Infection 17:355-359.

Presutti, D. G., and H. M. Hassan. 1995. Binding of integration host factor (IHF) to the Escherichia coli sodA gene and its role in the regulation of a sodA-lacZ fusion gene. Mol. Gen. Genet. 246:228-235. 
Pugsley, A. P. 1993. The complete general secretory pathway in Gram-negative bacteria. Microbiol. Rev. 57:50-108.

Purser, J. E., and S. J. Norris. 2000. Correlation between plasmid content and infectivity in Borrelia burgdorferi. Proc. Natl. Acad. Sci. USA 25:13865-13870.

Qi, H. Y., and H. D. Bernstein. 1999. SecA is required for the insertion of inner membrane proteins targeted by the Escherichia coli signal recognition particle. J. Biol. Chem. 274:8993-8997.

Rabilloud, T. 1992. Silver staining with silver nitrate. Electrophoresis 13:429-439.

Raibaud, O., and M. Schwartz. 1984. Positive control of transcription initiation in bacteria. Annu. Rev. Genet. 18:173-206.

Rajapandi, T., K. Dolan, and D. Oliver. 1991. The first gene in the Escherichia coli secA operon, gene $\mathrm{X}$, encodes a nonessential secretory protein. J. Bacteriol. 173:70927097.

Reddy, K. N., and G. Markus. 1972. Mechanism of activation of human plasminogen by streptokinase. Presence of active center in streptokinase-plasminogen complex. J. Biol. Chem. 247:1683-1691.

Reissbrodt, R., R. Kingsley, W. Rabsch, W. Beer, M. Roberts, and P. H. Williams. 1997. Iron-regulated excretion of $\alpha$-keto acids by Salmonella typhimurium. J. Bacteriol. 179:4538-4544.

Rich, S. M., P. M. Armstrong, R. D. Smith, and S. R. Telford. 2001. Lone star tickinfecting borreliae are most closely related to the agent of bovine borreliosis. J. Clin. Microbiol. 39:494-497.

Richardson, K., J. Michalski, and J. B. Kaper. 1986. Hemolysin production and cloning of two hemolysin determinants from classical Vibrio cholerae. Infect. Immun. 54:415420.

Rittig, M. G., T. Haupl, A. Krause, M. Kressel, P. Groscurth, and G. R. Burmester. 1994. Borrelia burgdorferi-induced ultrastructural alterations in human phagocytes: a clue to pathogenicity? J. Pathol. 173: 269-282. 
Rittig, M. G., J. C. Jagoda, B. Wilske, R. Murgia, M. Cinco, R. Repp, G. R. Burmester, and A. Krause. 1998. Coiling phagocytosis discriminates between spirochetes and is enhanced by phorbol myristate acetate and granulocyte-macrophage colonystimulating factor. Infect. Immun. 66:627-635.

Rittig M. G., A. Krause, T. Haupl, U. E. Schaible, M. Modolell, M. D. Kramer, E. Lutjen-Drecoll, M. M. Simon, and G. R. Burmester. 1992. Coiling phagocytosis is the preferential phagocytic mechanism for Borrelia burgdorferi. Infect. Immun. 60:4205-4212.

Rosa, P. A., D. S. Samuels, D. Hogan, B. Stevenson, S. Casjens, and K. Tilly. 1996. Directed insertion of a selectable marker into a circular plasmid of Borrelia burgdorferi. J. Bacteriol. 178:5946-5953.

Rosa, P. A., T. Schwan, and D. Hogan. 1992. Recombination between genes encoding major outer surface proteins A and B of Borrelia burgdorferi. Mol. Microbiol. 6:3031-3040.

Rosenfeld J., J. Capdevielle, J. C. Guillemot, and P. Ferrara. 1992. In-gel digestion of proteins for internal sequence analysis after one- or two-dimensional gel electrophoresis. Anal. Biochem. 2203:173-179.

Rossbach, S. T. L. Wilson, M. L. Kukuk, and H. A. Carty. 2000. Elevated zinc induces siderophores biosynthesis genes and a zntA-like gene in Pseudomonas fluorescens. FEMS Microbiol. Lett. 191:61-70.

Rotilio, G. R., R. C. Bray, and E. M. Gielden. 1972. A pulse radiolysis study of superoxide dismutase. Biochim. Biophys. Acta 268:605-609.

Ruzic-Sabljic, F. Strle, J. Cimperman, V. Maraspin, S. Lotric-Furlan, and D. PleterskiRigler. 2000. Characterization of Borrelia burgdorferi sensu lato strains isolated from patients with skin manifestations of Lyme borreliosis residing in Slovenia. J. Med. Microbiol. 49:47-53.

Sadziene, A., P. A. Thompson, and A. G. Barbour. 1993. In vitro inhibition of $B$. burgdorferi growth by antibodies. J. Infect. Dis. 167:165-167.

Salavati, R., and D. B. Oliver. 1995. Competition between ribosome and SecA binding promotes Escherichia coli secA translational regulation. RNA 1:745-753. 
Sambrook, J., E. F. Fritsch, and T. Maniatis. 1989. Molecular cloning: a laboratory manual, 2nd ed. Cold Spring Harbor Laboratory Press, Cold Spring Harbor, New York.

Samuels, D. S., and C. F. Garon. 1993. Coumermycin $A_{1}$ inhibits growth and induces relaxation of supercoiled plasmids in Borrelia burgdorferi, the Lyme disease agent. Antimicrob. Agents. Chemother. 37:46-50.

Samuels, D. S., K. E. Mach, and C. F. Garon. 1994. Genetic transformation of the Lyme disease agent Borrelia burgdorferi with coumarin-resistant gyrB. J. Bacteriol. 176:6045-6049.

Samuels, D. S., R. T. Marconi, and C. F. Garon. 1993. Variation in the size of the ospAcontaining linear plasmid, but not the linear chromosome, among the three Borrelia species associated with Lyme disease. J. Gen. Microbiol. 139:2445-2449.

Sanders, D. A., B. L. Gillece-Castro, A. L. Burlingame, and D. E. Koshland. 1992. Phosphorylation site of NtrC, a protein phosphatase whose covalent intermediate activates transcription. J. Bacteriol. 174:5117-5122.

Sanger, F., S. Nicklen, and A. R. Coulson. 1977. DNA sequencing with chain-terminating inhibitors. Proc. Natl. Acad. Sci. USA 74:5463-5467.

SanMateo, L. R., M. M. Hobbs, and T. H. Kawula. 1998a. Periplasmic copper-zinc superoxide dismutase protects Haemophilus ducreyi from exogenous superoxide. Mol. Microbiol. 27:391-404.

SanMateo, L. R., K. L. Toffer, and T. H. Kawula. 1998b. The sodA gene of Haemophilus ducreyi encodes a hydrogen peroxide-inhibitable superoxide dismutase. Gene 207:251-257.

Sarker, S., K. E. Rudd, and D. B. Oliver. 2000. Revised translation start site for secM defines an atypical signal peptide that regulates Escherichia coli secA expression. J. Bacteriol. 182:5592-5595.

Sato, Y., K. Miyamoto, A. Iwaki, T. Masuzawa, Y. Yanagihara, E. I. Korenberg, N. B. Gorelova, V. I. Volkov, L. I. Ivanov, and R. N. Liverova. 1996. Prevalence of 
Lyme disease spirochetes in Ixodes persculatus and wild rodents in far eastern

Russia. Appl. Environ. Microbiol. 62:3887-3889.

Schaible, U. E., M. D. Kramer, and K. Eichmann. 1990. Monoclonal antibodies specific for the outer surface protein A (OspA) of Borrelia burgdorferi prevent Lyme borreliosis in severe combined immunodeficiency (Scid) mice. Proc. Natl. Acad. Sci. USA 92:2909-2912.

Schaumann, R., V. Fingerle, K. Buchholz, F. B. Spencker, and A. C. Rodloff. 1999. Facial palsy caused by Borrelia infection in a twin pregnancy in an area of nonendemicity. Clin. Infect. Dis. 29:955-956.

Schmiel, D. H., and V. I. Miller. 1999. Bacterial phospholipases and pathogenesis. Microbes Infect. 1:1103-1112.

Schrock, C. G. 1982. Lyme disease: additional evidence of widespread distribution. Recognition of a tick-borne dermatitis syndrome in an area of known Ixodes tick distribution. Am. J. Med. 72:700-706.

Schwan, T. G., W. Burgdorfer, and C. F. Garon. 1988. Changes in infectivity and plasmid profile of the Lyme disease spirochete, $B$. burgdorferi, as a result of in vitro cultivation. Infect. Immun. 56:1831-1836.

Schwan, T. G., and J. Piesman. 2000. Temporal changes in outer surface proteins A and C of the Lyme disease-associated spirochete, Borrelia burgdorferi, during the chain of infection in ticks and mice. J. Clin. Microbiol. 38:382-388.

Schwan, T. G., J. Piesman, W. T. Golde, M. C. Dolan, and P. A. Rosa. 1995. Induction of an outer surface protein on Borrelia burgdorferi during tick feeding. Proc. Natl. Acad. Sci. USA 92:2909-2913.

Seiler, K. P., Z. Vavrin, E. Eichwald, J. B. Hibbs, and J. J. Weis. 1995. Nitric oxide production during murine Lyme disease: lack of involvement in host resistance or pathology. Infect. Immun. 63:3886-3895.

Sellati, T. J., L. D. Abrescia, J. D. Radolf, and M. B. Furie. 1996. Outer surface lipoproteins of Borrelia burgdorferi activate vascular endothelium in vitro. Infect. Immun. 64:3180-3187. 
Sellati, T. J., D. A. Bouis, M. J. Caimano, J. A. Feulner, C. Ayers, E. Lien, and J. D. Radolf. 1999. Activation of human monocytic cells by Borrelia burgdorferi and Treponema pallidum is facilitated by CD14 and correlates with surface exposure of spirochetal lipoproteins. J. Immunol. 163:2049-2056.

Shapiro, E. D., and M. A. Gerber. 2000. Lyme disease. Clin. Infect. Dis. 31:533-542.

Sigal, L. H. 1994. Persisting symptoms of Lyme disease-possible explanations and implications for treatment. J. Rheumatol. 21:593-595.

Sigal, L. H. 1997. Lyme disease: a review of aspects of its immunology and immunopathogenesis. Annu. Rev. Immunol. 15:63-92.

Simonen, M., and I. Palva. 1993. Protein secretion in Bacillus species. Microbiol. Rev. 57:109-137.

Simpson, W. J., W. Cieplak, M. E. Schrumpf, A. G. Barbour, and T. G. Schwan. 1994. Nucleotide sequence and analysis of the gene in Borrelia burgdorferi encoding the immunogenic P39 antigen. FEMS Microbiol. Lett. 119:381-388.

Simpson, W. J., C. F. Garon, and T. G. Schwan. 1990. Analysis of supercoiled circular plasmids in infectious and non-infectious Borrelia burgdorferi. Microb. Pathogen. 8:109-118.

Singh, V. K., A. Xiong, T. R. Usgaard, S. Chakrabarti, R. Deora, T. K. Misra, and R. K. Jayaswal. 1999. ZntR is an autoregulatory protein and negatively regulates the chromosomal zinc resistance operon, znt, of Staphylococcus aureus. Mol. Microbiol. 33:200-207.

Smith, T. L. 1998. Oxidative stress response of Borrelia burgdorferi. Thesis. University of Louisville, Louisville, Kentucky.

Sodeinde, O. A., A. K. Sample, R. R. Brubaker, and J. D. Goguen. 1988. Plasminogen activator/coagulase gene of Yersinia pestis is responsible for degradation of plasmidencoded outer membrane proteins. Infect. Immun. 56:2749-2752. 
Sohaskey, C. D., C. Arnold, and A. G. Barbour. 1997. Analysis of promoters in Borrelia burgdorferi by use of a transiently expressed reporter gene. J. Bacteriol. 179:68376842.

Stafford, K. C., R. F. Massung, L. A. Magnarelli, J. W. Ijdo, and J. F. Anderson. 1999. Infection with agents of human granulocytic ehrlichiosis, Lyme disease, and babesiosis in wild white-footed mice (Peromyscus leucopus) in Connecticut. J. Clin. Microbiol. 37: 2887-2892.

Stamm, L. V., and N. W. Charon. 1979. Plate assay for detection of Leptospira interrogans serovar pomona hemolysin. J. Clin. Microbiol. 10:590-592.

Stamm, L. V., F. C. Gherardini, E. A. Parrish, and C. R. Moomaw. 1991. Heat shock response of spirochetes. Infect. Immun. 4:1572-1575.

Steere, A. C., and S. E. Malawista. 1979. Cases of Lyme disease in the United States: locations correlated with distribution of Ixodes dammini ticks. Ann. Intern. Med. 91:730-733.

Steere, A. C., S. E. Malawista, D. R. Snydman, R. E. Shope, W. A. Andiman, M. R. Rosi, and F. M. Steele. 1977. Lyme arthritis: an epidemic of oligoarticular arthritis in children and adults in three Connecticut communities. Arth. Rheum. 20:7-17.

Steere, A. C., V. K. Sikand, F. Meurics, D. L. Parenti, E. Fikrig, and R. T. Schoen. 1998. Vaccination against Lyme disease with recombinant Borrelia burgdorferi outersurface lipoprotein A with adjuvant. New Engl. J. Med. 339:209-215.

Stevenson, B., K. Tilly, and P. A. Rosa. 1996. A family of genes located on four separate 32-kilobase circular plasmids in Borrelia burgdorferi B31. J. Bacteriol. 178:35083516.

Stohs, S. J., and D. Bagchi. 1995. Oxidative mechanisms in the toxicity of metal ions. Free Rad. Biol. Med. 18:321-336.

Stojiljkovic, I., A. J. Baumler, and K. Hantke. 1994. Fur regulon in Gram-negative bacteria: identification and characterization of new iron-regulated Escherichia coli genes by a Fur titration assay. J. Mol. Biol. 236:531-545. 
Storz, G., L. A. Tartaglia, and B. N. Ames. 1990a. Transcriptional regulator of oxidative stress-inducible genes: direct activation by oxidation. Science 248:189-194.

Storz, G., L. A. Tartaglia, S. B. Farr, and B. N. Ames. 1990b. Bacterial defenses against oxidative stress. Trends Gen. 6:363-368.

Storz, G., and M. B. Toledano. 1994. Regulation of bacterial gene expression in response to oxidative stress. Meth. Enzymol. 236:196-207.

Strle F., J. A. Nelson, E. Ruzic-Sabljiic, J. Cimperman, V. Maraspin, S. Lotric-Furlan, Y. Cheng, M. M. Picken, G. M. Trenholme, and R. N. Picken. 1996. European Lyme borreliosis: 231 culture-confirmed cases involving patients with erythema migrans. Clin. Infect. Dis. 23:61-65.

Studholme, D. J., and M. Buck. 2000. The biology of enhancer-dependent transcriptional regulation in bacteria: insights from genome sequences. FEMS Microbiol. Lett. 186:1-9.

Suh, S. J., L. Silo-Suh, D. E. Woods, D. J. Hassett, S. E. West, and D. E. Ohman. 1999. Effect of rpoS mutation on the stress response and expression of virulence factors in Pseudomonas aeruginosa. J. Bacteriol. 181:3890-3897.

Suhonen, J., K. Hariala, and M. K. Viljanen. 1998. Tube phagocytosis, a novel way for neutrophils to phagocytize Borrelia burgdorferi. Infect. Immun. 66:3433-3435.

Svensater, G., B. Sjogreen, and I. R. Hamilton. 2000. Multiple stress responses in Streptococcus mutans and the induction of general and stress-specific proteins. Microbiol. 146:107-117.

Tai, S. P. S., A. E. Krafft, P. Noothet, and R. K. Holmes. 1990. Coordinate regulation of siderophore and diphtheria toxin production by iron in Corynebacterium diptheriae. Microb. Pathogen. 9:267-273.

Takayama, K., R. J. Rothenberg, and A. G. Barbour. 1987. Absence of lipopolysaccharide in the Lyme disease spirochete, Borrelia burgdorferi. Infect. Immun. 48:2311-2313. 
Talkington, J. and S. P. Nickell. 1999. Borrelia burgdorferi spirochetes induce mast cell activation and cytokine release. Infect. Immun. 67:1107-1115.

Talkington, J. and S. P. Nickell. 2001. Role of Fc gamma receptors in triggering host cell activation and cytokine release by Borrelia burgdorferi. Infect. Immun. 69:413-419.

Tao, H., C. Bausch, C. Richmond, F. R. Blattner, and T. Conway. 1999. Functional genomics: expression analysis of Escherichia coli growing on minimal and rich media. J. Bacteriol. 181:6425-6440.

Tatro, J. B., L. I. Romero, D. Beasley, A. C. Steere, and S. Reichlin. 1994. Borrelia burgdorferi and Escherichia coli lipopolysaccarides induce nitric oxide and interleukin-6 production in cultured rat brain cells. J. Infect. Dis. 169:1014-1022.

Taylor, J. P., G. R. Istre, T. C. McChesney, F. T. Satalowich, R. L. Parker, and L. M. McFarland. 1991. Epidemiologic characteristics of human tularemia in the southwest-central states, 1981-1987. Am. J. Epidemiol. 133:1032-1038.

Tilly, K., S. Casjens, B. Stevenson, J. L. Bono, D. S. Samuels, D. Hogan, and P. Rosa. 1997. The Borrelia burgdorferi circular plasmid cp26: conservation of plasmid structure and targeted inactivation of the $\operatorname{sp} C$ gene. Mol. Microbiol. 25:361-373.

Tilly, K., R. Hauser, J. Campbell, and G. J. Ostheimer. 1993. Isolation of dnaJ, dnaK, and grpE homologues from Borrelia burgdorferi and complementation of Escherichia coli mutants. Mol. Microbiol. 7:359-369.

Tuveson, R. W., and R. B. Jonas. 1979. Genetic control of near-UV (300-400 nm) sensitivity independent of the recA gene in strains of Escherichia coli K12. Photochem. Photobiol. 30:667-676.

Uhl, B., B. Wolf, J. Schwinde, A. Metzger, G. Jung, W. G. Bessler, and S. Hauschildt. 1991. Intracellular localization of a lipopeptide macrophage activator: immunocytochemical investigations and EELS analysis on ultra-thin cryosections of bone marrow-derived macrophages. J. Leukocyte Biol. 50:10-18.

vanDam, A. P., H. Kuiper, K. Vos, A. Widjojokusumo, B. M. de Jongh, L. Spanjaard, A. C. Ramselaar, M. D. Kramer, and J. Dankert. 1993. Different genospecies of Borrelia burgdorferi are associated with distinct clinical manifestations of Lyme borreliosis. Clin. Infect. Dis. 17:708-717. 
vanVliet, A. H., M. A. Baillon, C. W. Penn, and J. M. Ketley. 1999. Campylobacter jejuni contains two Fur homologs: characterization of iron-responsive regulation of peroxide stress defense genes by the PerR repressor. J. Bacteriol. 181:6371-6376.

vanVliet, A. H., K. G. Woodridge, and J. M. Ketley. 1998. Iron-responsive gene regulation in a Campylobacter jejuni fur mutant. J. Bacteriol. 180:5291-5298.

Walker, D. H. 1998. Tick-transmitted infectious diseases in the United States. Annu. Rev. Public Health. 19:237-269.

Walker, D. H., and J. S. Dumler. 1996. Emergence of the ehrlichioses as human health problems. Emerging Inf. Dis. 2:18-26.

Walker, J., M. Saraste, M. Runswick, and N. Gay. 1982. Distantly related sequences in the alpha- and beta-subunits of ATP synthase, myosin, kinases, and other ATPrequiring enzymes and a common nucleotide binding fold. EMBO J. 8:945-951.

Wang, G., A. P. vanDam, and J. Dankert. 1999a. Evidence of frequent ospC gene transfer between Borrelia valaisiana sp. nov. and other Lyme disease spirochetes. FEMS Microbiol. Lett. 177:289-296.

Wang, G., A. P. vanDam, I. Schwartz, and J. Dankert. 1999b. Molecular typing of B. burgdorferi sensu lato: taxonomic, epidemiological, and clinical implications. Clin. Microbiol. Rev. 12:633-653.

Wang, I. N., D. L. Smith, and R. Young. 2000. Holins: the protein clocks of bacteriophage infections. Annu. Rev. Microbiol. 54:799-825.

Weis, J. J., L. Yang, K. P. Seiler, and R. M. Silver. 1997. Pathological manifestations in murine Lyme disease: association with tissue invasion and spirochete persistence. Clin. Infect. Dis. 25:S18-S24.

Weisbach, H., and N. Bsat. 1991. Regulation of methionine synthesis in Escherichia coli. Mol. Microbiol. 5:1593-1597.

Westermeier, R. 1997. Electrophoresis in practice. Wiley Publishing. Weinheim, Germany. 
Whitehouse, C. 1997. Identification and molecular characterization of a superoxide dismutase of Borrelia burgdorferi. Dissertation. University of Louisville, Louisville, Kentucky.

Whitehouse, C. A., L. R. Williams, and F. E. Austin. 1997. Identification of superoxide dismutase activity in Borrelia burgdorferi. Infect Immun. 65:4865-4868.

Williams, L. R. and F. E. Austin. 1992. Hemolytic activity of Borrelia burgdorferi. Infect. Immun. 3224-3230.

Wise, A., R. Brems, V. Ramakrishnan, and M. Villarejo. 1996. Sequences in the -35 region of Escherichia coli rpoS-dependent genes promote transcription by $\sigma^{\mathrm{S}}$. J. Bacteriol. 178:2785-2793.

Wolf, S. G., D. Frenkiel, T. Arad, S. E. Finkel, R. Kolter, and A. Minsky. 1999. DNA protection by stress-induced biocrystallization. Nature 400:83-85.

Wooten, R. M., V. R. Modur, T. M. McIntyre, and J. J. Weis. 1996. Borrelia burgdorferi outer membrane protein A induces nuclear translocation of nuclear factor- $\kappa \beta$ and inflammatory activation in human endothelial cells. J. Immunol. 157:4584-4590.

Wooten, R. M., R. B. Morrison, J. H. Weis, S. D. Wright, R. Thieringer, and J. J. Weis. 1998. The role of CD14 in signaling mediated by outer membrane lipoproteins of Borrelia burgdorferi. J. Immunol. 160:5485-5492.

World Health Organization. (1996). Report of WHO workshop on Lyme Borreliosis Diagnosis and Surveillance. Warsaw, Poland. WHO/CDS/VPH:141-142.

Xiong, A., V. K. Singh, G. Cabrera, and R. K. Jayaswal. 2000. Molecular characterization of the ferric-uptake regulator, fur, from Staphylococcus aureus. Microbiol. 146:659-68.

Xu, Y., and R. C. Johnson. 1995. Analysis and comparison of plasmid profiles of Borrelia burgdorferi sensu lato strains. J. Clin. Microbiol. 33:2679-2685. 
Xu, Y., C. Kodner, L. Coleman, and R. C. Johnson. 1996. Correlations of plasmids with infectivity of Borrelia burgdorferi sensu stricto type strain B31. Infect. Immun. 64:3870-3876.

Yost, F. J., and I. Fridovich. 1973. An iron-containing superoxide dismutase from Escherichia coli. J. Biol. Chem. 248:4905-4908.

Zhang, Y., R. Lathigra, T. Garbe, D. Catty, and D. Young. 1991. Genetic analysis of superoxide dismutase, the 23-kilodalton antigen of Mycobacterium tuberculosis. Mol. Microbiol. 5:381-391.

Zheleznova, E. E., J. H. Crosa, and R. G. Brennan. 2000. Characterization of the DNAand metal-binding properties of Vibrio anguillarum Fur reveals conservation of a structural $\mathrm{Zn}^{2+}$ ion. J. Bacteriol. 182:6264-6267.

Zheng, M., B. Doan, T. D. Schneider, and G. Storz. 1999. OxyR and SoxRS regulation of fur. J. Bacteriol. 181:4639-3643

Zuckert, W. R., and J. Meyer. 1996. Circular and linear plasmids of Lyme disease spirochetes have extensive homology: characterization of a repeated DNA element. J. Bacteriol. 178:2287-2298. 


\section{VITA}

The author, Tonya Lynn Nichols was born on August 12,1964 and was raised in Elmore County, Alabama. She graduated Valedictorian from Elmore County High School in 1982. In September of that year, she entered Livingston University in Livingston, Alabama. She received the degree of Bachelor of Science with a major in Biology and a minor in Chemistry.

After graduation, Tonya attended the United States Army’s Officer Candidate School at Fort Benning, Georgia. In April of 1987, she was commissioned as a Lieutenant in the United States Army’s Adjutants General Corps. Her follow-on military assignments have included Human Relations training at Fort Benjamin Harrison, Indiana and Nuclear, Biological and Chemical training at Fort Hood, Texas. She served as a Platoon Leader in the largest heavy equipment maintenance company in the Texas National Guard.

In 1991, she received a Masters of Science in Biology from Baylor University in

Waco, Texas. While attending school at Baylor, she concurrently worked as a laboratory technician on a research project investigating a prognostic indicator for prostate cancer. It was during this time that she earned her certification in Immunology by the American Society of Clinical Pathology. From 1993 through 1996 Tonya worked as an Assistant Professor at Columbus State University, Columbus Georgia.

In 1996 she entered the PhD program in the Department of Microbiology and Immunology at the University of Louisville, Louisville Kentucky. Upon graduation, Tonya will begin a postdoctoral research fellowship at Procter \& Gamble Pharmaceuticals in Mason, Ohio.

Tonya and her husband, Greg, have two boys, Matthew and Joshua Nichols 